\title{
Toward Picard-Lefschetz Theory of Path Integrals, Complex Saddles and Resurgence
}

\author{
Alireza Behtash,,$^{a, c}$ Gerald V. Dunne, ${ }^{b}$ Thomas Schäfer, $^{a}$ Tin Sulejmanpasic, $^{a}$ \\ Mithat Ünsal ${ }^{a, c}$ \\ ${ }^{a}$ Department of Physics, North Carolina State University, Raleigh, NC 27695, USA \\ ${ }^{b}$ Department of Physics, University of Connecticut, Storrs, CT 06269-3046, USA \\ ${ }^{c}$ Department of Mathematics, Harvard University, Cambridge, MA, 02138, USA
}

\begin{abstract}
We show that the semi-classical analysis of generic Euclidean path integrals necessarily requires complexification of the action and measure, and consideration of complex saddle solutions. We demonstrate that complex saddle points have a natural interpretation in terms of the Picard-Lefschetz theory. Motivated in part by the semi-classical expansion of QCD with adjoint matter on $\mathbb{R}^{3} \times S^{1}$, we study quantum-mechanical systems with bosonic and fermionic (Grassmann) degrees of freedom with harmonic degenerate minima, as well as (related) purely bosonic systems with harmonic non-degenerate minima. We find exact finite action non-BPS bounce and bion solutions to the holomorphic Newton equations. We find not only real solutions, but also complex solution with non-trivial monodromy, and finally complex multi-valued and singular solutions. Complex bions are necessary for obtaining the correct non-perturbative structure of these models. In the supersymmetric limit the complex solutions govern the ground state properties, and their contribution to the semiclassical expansion is necessary to obtain consistency with the supersymmetry algebra. The multi-valuedness of the action is either related to the hidden topological angle or to the resurgent cancellation of ambiguities. We also show that in the approximate multi-instanton description the integration over the complex quasi-zero mode thimble produces the most salient features of the exact solutions. While exact complex saddles are more difficult to construct in quantum field theory, the relation to the approximate thimble construction suggests that such solutions may be underlying some remarkable features of approximate bion saddles in quantum field theories.
\end{abstract}




\section{Contents}

1 Introduction and physical motivation from QFT 2

1.1 Complex Saddle Points in Complexified Euclidean Path Integrals 3

1.2 Motivation from QFT translated to quantum mechanics 6

1.3 Necessity of complexification: Simple pictures 7

1.4 What is surprising (and what is not)? 12

1.5 Holomorphic Newton equations 14

1.6 Relation to complex gradient flow and Picard-Lefschetz theory 16

2 Graded Hilbert spaces $\quad 19$

2.1 Supersymmetric QM and its non-supersymmetric generalization 19

2.2 Fermionic Fock space 20

2.3 Graded Hilbert space: Fermion number representation 22

2.4 Derivation from the fermionic determinant 23

2.5 Graded Hilbert space: Spin representation 25

2.6 Twisted partition function vs. supersymmetric Witten index 27

2.7 Isospectral pairs 28

3 Original formulation and local-global relations 30

3.1 Auxiliary Potential 30

3.2 Local harmonic analysis, states, and auxiliary Morse function 31

3.3 Instantons and index theorem for Dirac operator 33

3.4 Difference between QFT and QM: Hilbert's hotel or not 35

3.5 Mixing of harmonic states in QM with fermions: New instanton effects 37

4 Approximate versions of exact solutions from quasi-zero mode thimbles $\quad 42$

4.1 Multi-instantons and boson and fermion induced interactions 42

$4.2[\mathcal{I} \mathcal{I}]$ thimble integration and approximate form of real bion 43

$4.3[\mathcal{I} \overline{\mathcal{I}}]$ thimble integration and approximate form of complex bion $\quad 45$

4.4 Other aspects of thimble integration 46

5 Graded formulation: Double-well potential 47

5.1 Quantum modified potential and exact non-BPS solutions 47

5.2 Exact (real) bounce solution $\quad 51$

5.3 Exact complex bion solution $\quad 54$ 
5.4 Actions and amplitudes of the (real) bounce and the complex bion $\quad 56$

5.5 Analytic continuation and monodromy: From real bounce to complex bion 58

5.6 Vacuum as a complex bion gas

6 Graded formulation: Periodic Sine-Gordon potential 63

6.1 Quantum modified potential and exact non-BPS solutions 64

6.2 Exact (real) bounce solution 65

6.3 Exact real bion solution $\quad 67$

6.4 Exact complex bion solution $\quad 69$

6.5 Analytic continuation: From real bounce to complex bion 72

6.6 Physical Role of Singular, Multi-valued Solutions $\quad 75$

6.7 Physics of the complex bion solution, multi-valuedness and singularity 77

6.8 Vacuum as a complex and real bion gas 81

6.9 Remark on fluctuation operators 84

7 Complex Saddles and Resurgence $\quad 85$

8 Comments on the literature $\quad 87$

8.1 Witten and Harlow-Maltz-Witten on analytic continuation in ChernSimons and Liouville theory $\quad 87$

8.2 Brezin, Le Guillou, and Zinn-Justin on complex instantons 89

8.3 Balitsky and Yung on complex quasi-solutions 90

$\begin{array}{lll}8.4 & \text { Other related works } & 90\end{array}$

9 Conclusion $\quad 91$

9.1 Prospects in quantum field theories $\quad 92$

9.2 Other Related Directions 94

$\begin{array}{ll}\text { A Review of some relevant elliptic integrals } & 96\end{array}$

B Absence of a Hidden Topological Angle in one-dimensional real inte$\begin{array}{lr}\text { grals with real parameters } & 97\end{array}$ 


\section{Introduction and physical motivation from QFT}

In this paper we pursue two goals, one related to the proper formalism of path integration in semi-classical analysis, and the other related to novel phenomena in quantum mechanics and quantum field theory associated with new semi-classical contributions. Our first aim is to argue that the correct framework for studying the semi-classical representation of a Euclidean path integral necessarily involves complexifying the configuration space, measure and action. This obviously implies complexification of classical mechanics arising from the Euclidean action. Typically, once this is done, there are complex saddle configurations, solutions to the complexified equations of motion. Our second goal is to elucidate the physical role of complex saddle configurations in the path integral formulation of quantum mechanics and quantum field theory. The examples reveal surprising new phenomena, with implications that force us to re-consider our intuition about the semi-classical approach to path integrals.

The motivation for the present study comes from an intuition that developed in supersymmetric and non-supersymmetric quantum field theories, in particular semiclassical studies of QCD with $N_{f}$ adjoint fermion on $\mathbb{R}^{3} \times S^{1}$ [1-4]. The case $N_{f}=1$ is $\mathcal{N}=1$ supersymmetric gauge field theory. In this context, monopole-instantons are solutions to the BPS (self-duality) equations, and magnetic and neutral bions, correlated 2-events, are manifestly non-self-dual, and not a solution to first order BPS equations of motion. However, bions have an interesting property that suggests that they may in fact be associated with exact saddle points of the path integral. Bions have a calculable characteristic size, $r_{b}$, parametrically larger than the monopole-instanton radius $r_{m}$ ( $r_{m}$ is dictated by the scale of the Higgs phenomenon of the Wilson line). The scale $r_{b} \sim r_{m} /\left(g^{2} N_{f}\right)$ determines the critical point of the quasi-zero mode integration. Neither $g^{2}$ nor $N_{f}$ enters the second order Yang-Mills equations of motion, and hence, bions are not solutions of the second order Euclidean equation.

A critical point on the quasi-zero mode integration contour at a finite separation (either real or complex) is an unusual property. In the standard textbook treatment of instantons in bosonic systems, it is shown that for the quasi-zero mode associated with the instanton anti-instanton separation the critical point is at infinite separation, reflecting the fact that the superposition of an instanton with an anti-instanton is not a solution at finite separation. This is related to the non-linearity of the underlying instanton equations, see the discussion in textbook [5-10] or reviews [11-13] on the subject. This raises the question of what the meaning of a critical point at finite (real or complex) quasi-zero mode separation is. In particular, is it possible that these configurations are exact saddle points in some suitable formalism, and not just approximate descriptions? What is the distinction between a critical point at real separation and at 
complex "separation"? Since the quasi-zero mode direction is a particular direction in field space, real separation is a real field configuration and complex direction is a complexified field configuration. What then is the natural habitat for these saddle points? What is the role of complex fields in the semi-classical treatment of real path integrals?

At this point in time we cannot provide definitive answers to all these questions in quantum field theory. However, attacking the quantum field theory problem in the more tractable context of quantum mechanics, we study quantum systems with one bosonic and $N_{f}$ fermionic (Grassmannian) fields, corresponding to a particle with internal spin $\left(\frac{1}{2}\right)^{N_{f}} . \quad N_{f}=1$ corresponds to the $\mathcal{N}=1$ supersymmetric quantum theory. In each case we demonstrate that both real and complex non-self dual saddle configurations exist, and that they solve complexified equations of motion which can be obtained by integrating out fermions exactly. ${ }^{1}$ We show that they must be included in a consistent semi-classical expansion of the quantum path integrals. In certain limits the exact solution has an approximate description in terms of instanton-anti-instanton correlated 2-events with real and complex quasi-zero mode separation. This PicardLefschetz thimble interpretation generalized more directly to quantum field theory, and suggests that even in quantum field theories exact complex saddle points may be underlying the known bion analysis [1-4, 14-18].

\subsection{Complex Saddle Points in Complexified Euclidean Path Integrals}

The basic question is the following: Given an ordinary path integral over real fields in a general Euclidean version of QFT or quantum mechanics,

$$
Z_{\mathrm{bos}}=\int D x(t) e^{-\frac{1}{\hbar} S[x(t)]}=\int D x(t) e^{-\frac{1}{\hbar} \int d t\left(\frac{1}{2} \dot{x}^{2}+V(x)\right)}
$$

how does one perform the semi-classical expansion? The same question also applies to theories with both bosonic and fermionic degrees of freedom.

This question is usually answered by studying real saddle points, single-valued and smooth configurations, such as instantons or multi-instantons [5-13]. However, we show in this work that important physics is missed in the standard approach which involves only real-valued "instanton" solutions. These solutions satisfy Newton's equation in the inverted potential $-V(x)$ :

$$
\frac{\delta S}{\delta x}=0 \Longrightarrow \frac{d^{2} x}{d t^{2}}=+\frac{\partial V}{\partial x} .
$$

\footnotetext{
${ }^{1}$ Although we are mostly motivated by theories with fermions, it is important to keep in mind that these systems can be represented in terms of purely bosonic systems after quantizing fermions, and projecting on fermion number eigenstates, and that all the conclusions of this work are equally valid for purely bosonic theories with generic (non-symmetric) bosonic potentials.
} 
Instantons and anti-instantons are solutions of the second order equations of motion by virtue of the real first-order equations:

$$
\frac{d x}{d t}= \pm \sqrt{2 V(x)}
$$

The two main new observations made in this work are:

1) In order to perform the semiclassical expansion in a Euclidean quantum mechanical path integral, the action and measure must be complexified. The partition function is

$$
Z_{\mathrm{bos}}=\int_{\Gamma} D z e^{-\frac{1}{\hbar} \mathcal{S}[z(t)]}=\int_{\Gamma} D z(t) e^{-\frac{1}{\hbar} \int d t\left(\frac{1}{2} \dot{z}^{2}+V(z)\right)},
$$

where $\Gamma$ is an integration cycle to be determined. Despite the fact that the original path integral is a sum over real configurations, the semi-classical expansion may receive physically important contributions from complex configurations. The critical points of the complexified path integral are found by solving the holomorphic Newton equations in the inverted potential $-V(z)$ :

$$
\frac{\delta \mathcal{S}}{\delta z}=0 \Longrightarrow \frac{d^{2} z}{d t^{2}}=+\frac{\partial V}{\partial z} .
$$

Clearly this provides a larger basis of classical configurations for the semi-classical expansion, and entails a physical interpretation in terms of Picard-Lefschetz theory [19-25]. We demonstrate that for generic potentials, physical properties of the ground state are determined by complex rather than real saddle points. We stress that this occurs in systems for which the physical couplings are manifestly real, so that the theories have a precise Hilbert space interpretation, which can be directly matched to the semi-classical analysis.

2) The generic saddle point configurations which contribute to the ground state properties of a general quantum mechanical system are complex solutions of the holomorphic Newton equations in the inverted potential, and they may even be multi-valued and singular. Despite this, the action of these saddles is finite, but typically with multi-valued imaginary part. The multi-valuedness and complexity of the action (recall that the path integral for real coupling must be real), may appear to be a serious problem, but it is indeed necessary. This phenomenon is related to either $i$ ) the resurgent cancellations with the (lateral) Borel resummation of perturbation theory, or $i$ ) the hidden topological angle phenomenon $[26,27]$. 
The former provides a rigorous version of the Bogomolny/Zinn-Justin (BZJ) prescription for controlling the ambiguities inherent in the analysis of instanton/antiinstanton correlated two-event amplitudes in the instanton gas picture in bosonic theories [28, 29].

In finite dimensional systems the analysis of exponential type integrals via the complex generalization of Morse theory, Picard-Lefschetz theory, and via resurgent trans-series are parallel constructions. Phenomena like Borel resummation, and the associated ambiguities from asymptotic analysis, find a geometric realization, respectively, as integration over Lefschetz thimbles and Stokes jumps of the thimbles. A discussion of multi-dimensional integrals, which defines a steepest surface using a complex gradient flow system, can be found in [19, 20]. In [21-24], this construction is implicit, and the saddle-point method is already based on the assumption of the appropriate Lefschetz thimble decomposition. The all-orders steepest descents analysis, which includes all contributions beyond the usual Gaussian approximation, reveals a rich and intricate structure of relations between the contributions from different saddles [22-24]. An application to infinite dimensional path integrals appeared in [25] in the context of Chern-Simons theory. In this case, due to the elliptic nature of the gradient flow system, the nice properties of the finite dimensional system carry over to the infinite dimensional case. Nevertheless, a surprising amount of this resurgent structure is also inherited by configuration space path integrals [30], for which the gradient flow system is parabolic. A number of recent talks by Kontsevich also emphasize the precise relation between resurgence and Lefschetz thimbles in finite dimensions, and discuss the extent to which these generalize to infinite dimensional problems [31-33]. The point of view he presents is close to our perspective, viewing the perturbative expansion around perturbative saddle points as a constructive approach to defining the path integral, even though the geometrization of the infinite dimensional path integral is more complicated and richer than the finite dimensional case. Kontsevich further claims that this framework could provide a rigorous mathematical replacement for path integrals, which is also an optimism that we share. We are also motivated by the well-known importance of complexified dynamics in the theory of algebraically integrable quantum systems [34-38].

From a complementary perspective, the uniform WKB approach provides a constructive and explicit realization of the relations between saddle points [39-41], similar to the finite dimensional relations. One finds that fluctuations about different nonperturbative multi-instanton sectors are related in a precise quantitative way, as has been confirmed recently by explicit quantum field theoretic multi-loop computations $[42,43]$. 
The perspective emanating from resurgence and finite dimensional examples of Lefschetz thimbles indicates that whenever we consider the semi-classical analysis of the path integral (either QFT or QM), we are required to start with a complexified/holomorphic version of it. In this paper, we explore consequences of this apparently innocuous step, which, in turn, leads to many surprises.

\subsection{Motivation from QFT translated to quantum mechanics}

The physical basis for the QFT intuition from studies of magnetic and neutral bions in $\mathrm{QCD}(\operatorname{adj})[1-4,14,15]$ and 2d non-linear sigma models with fermions [16-18, 44-47], translated to the quantum mechanical context with $N_{f}$ Grassmann valued (fermion) fields, amounts to the following. First, consider an instanton/instanton or an instanton/anti-instanton pair in the bosonic theory $\left(N_{f}=0\right)$. Since the BPS equations are non-linear, it is clear that a superposition of two individual solutions is not a solution at any finite separation $\tau$ between them. In fact, the action of the configuration changes with separation as

$$
S_{ \pm}(\tau) \equiv 2 S_{I}+\mathcal{V}_{ \pm}(\tau)=2 S_{I} \pm \frac{A a^{3}}{g} e^{-m_{b} \tau} \quad \text { bosonic models }
$$

where the + sign is for an $[\mathcal{I} \mathcal{I}]$ configuration, and the - sign for an $[\mathcal{I} \overline{\mathcal{I}}]$ configuration. Thus, the critical point of the potential between the two-events is at infinite separation, $\tau^{*}=\infty$, which we refer to as a "critical point at infinity".

This is the point where one first realizes that something may be special about bions in theories with fermions. For bions in such theories the potential between the constituent instantons has a critical point at a finite separation. ${ }^{2}$ For example, the potential between two instantons in QM is of the form:

$$
S_{ \pm}(\tau) \equiv 2 S_{I}+\mathcal{V}_{ \pm}(\tau)=2 S_{I} \pm \frac{A a^{3}}{g} e^{-m_{b} \tau}+N_{f} m_{b} \tau \quad \text { theories with fermions }
$$

and there exists a critical point at finite-separation $\tau^{*}<\infty$ :

$$
\begin{aligned}
& \frac{d \mathcal{S}_{+}(\tau)}{d \tau}=0 \quad \Rightarrow \quad m_{b} \tau^{*}=\log \left(\frac{A a^{3}}{g N_{f}}\right) \quad \text { for }[\mathcal{I I}], \\
& \frac{d \mathcal{S}_{-}(\tau)}{d \tau}=0 \quad \Rightarrow \quad m_{b} \tau^{*}=\log \left(\frac{A a^{3}}{g N_{f}}\right) \pm i \pi \quad \text { for }[\mathcal{I} \overline{\mathcal{I}}]
\end{aligned}
$$

\footnotetext{
${ }^{2}$ This is a universal phenomenon. (1.7) coincides with the quasi-zero-mode (QZM) integrations in QFTs, such as non-linear sigma models in $2 \mathrm{~d}$ and $\mathrm{QCD}(\mathrm{adj})[1,14,16-18,44]$, and $\mathcal{N}=1 \mathrm{SYM}$ in $4 \mathrm{~d}$ by a simple change of variables.
} 
The corresponding critical amplitude is

$$
\begin{aligned}
& \exp \left[-\frac{1}{g} S_{+}\right]=\left(\frac{g N_{f}}{A a^{3}}\right)^{N_{f}} e^{-2 S_{I}} \text { for }[\mathcal{I I}], \\
& \exp \left[-\frac{1}{g} S_{-}\right]=e^{ \pm i \pi N_{f}}\left(\frac{g N_{f}}{A a^{3}}\right)^{N_{f}} e^{-2 S_{I}} \text { for }[\mathcal{I} \overline{\mathcal{I}}] .
\end{aligned}
$$

The first case suggests that the $[\mathcal{I I}]$-two-event may actually be an approximate form of an exact real solution, while the latter case suggests that the $[\mathcal{I} \overline{\mathcal{I}}]$-two-event may be an approximate form of a complex (possibly multi-valued) exact solution. The factor $e^{ \pm i \pi N_{f}}$, where $N_{f}$ can also be continued to non-integer values, associated with the $[\mathcal{I} \overline{\mathcal{I}}]$ plays a crucial role in the behavior of supersymmetry in these fermion/boson systems. We show explicitly in Sections 5 and 6 that there are exact non-BPS saddle point solutions with size $\operatorname{Re}\left(\tau^{*}\right)$ in these two cases. See Fig.9.

Based on these results we demonstrate in quantum mechanics a precise relation between the nature of the integration over the complex quasi-zero mode (QZM) thimble and the existence of exact solutions of the full path integral:

i) $\tau^{*}=\infty \Longleftrightarrow$ Approximate quasi-solution.

ii) $\tau^{*} \in \mathbb{R}^{+}, \tau^{*}<\infty \Longleftrightarrow$ Exact real solution.

iii) $\tau^{*} \in \mathbb{C} \backslash \mathbb{R}^{+}, \operatorname{Re} \tau^{*}<\infty \Longleftrightarrow$ Exact complex solution.

We show that the integral over the QZM-thimble reproduces the most important features of the exact non-BPS solutions, such as the critical size, the ambiguous imaginary part of their action, and the hidden topological angle(s) associated with the exact solutions. Based on these, we conjecture that this deduction is also valid in general QFTs.

\subsection{Necessity of complexification: Simple pictures}

To demonstrate the necessity of considering complex solutions, we first provide some simple illustrations. We will show that the following quantum mechanical problems are closely related:

- Systems with degenerate harmonic minima coupled to $N_{f}$ Grassmann valued fields.

- Bosonic systems with non-degenerate harmonic minima. 


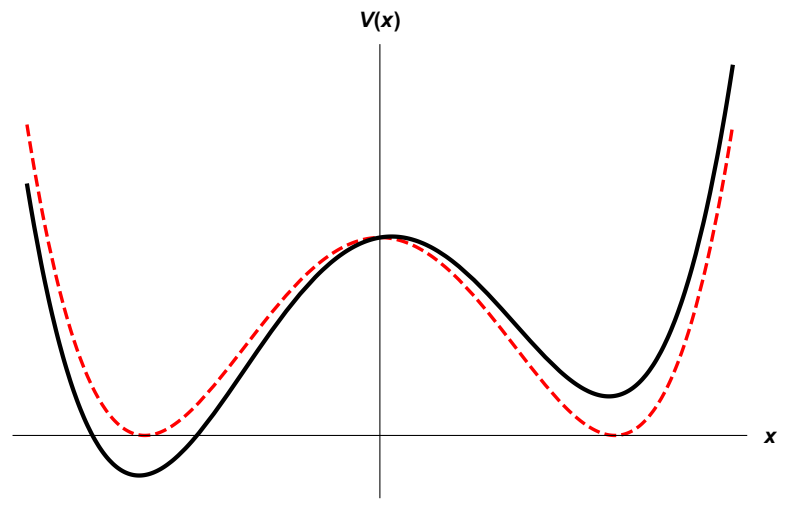

Figure 1. Bosonic potential $V_{\mathrm{bos}}(x)$ (red dashed) vs. quantum modified potential (black solid) $V(x)$ for double-well system. The quantum modified potential (1.11) is obtained upon quantizing the fermions and projecting to a fermion number (or spin) eigenstate.

In order to be specific, we consider two different bosonic potentials, a double-well potential and a periodic potential, see Fig 1 and 2. We couple Grassmann fields to these potentials and project on fermion number eigenstates. This leads to effective bosonic potentials in the sector with fermion number $p$

$$
V(x)=\underbrace{\frac{1}{2}\left(W^{\prime}(x)\right)^{2}}_{V_{\mathrm{bos}}(x)}+\frac{p g}{2} W^{\prime \prime}(x), \quad p=2 k-N_{f},
$$

which we refer to as the quantum modified potential. The fermion number $p$ is given by $p=2 k-N_{f}$ with $k=0, \ldots, N_{f}$. In a SUSY theory, with $N_{f}=1$, the function $W(x)$ is the superpotential $[48,49]$. For $N_{f}=2,3 \ldots$, we refer to $W(x)$ as an auxiliary potential.

In this sense, the first class of systems (with fermions) that we study can be expressed as a collection of systems of the second type. "Integrating out" fermions lifts the degeneracy of the harmonic minima. It is also useful to consider $p$ as an arbitrary parameter, which can even be taken to be complex.

The bosonic double-well (DW) potential, with $W(x)=\frac{1}{3} x^{3}-a^{2} x$, leads to a quantum modified tilted-double-well potential

$$
V_{ \pm}=\frac{1}{2}\left(x^{2}-a^{2}\right)^{2} \pm p g x
$$

Similarly, the bosonic periodic Sine-Gordon (SG) potential, with $W(x)=4 a^{3} \cos \left(\frac{x}{2 a}\right)$, leads to a quantum modified double-Sine-Gordon potential

$$
V_{ \pm}=2 a^{4} \sin ^{2}\left(\frac{x}{2 a}\right) \mp \frac{p g a}{2} \cos \left(\frac{x}{2 a}\right) \text {. }
$$




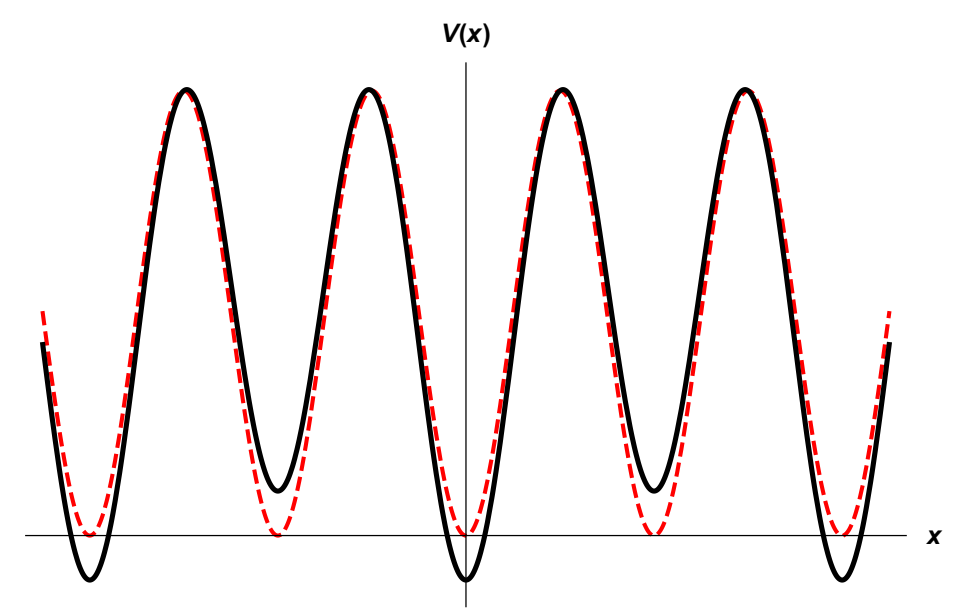

Figure 2. Bosonic potential $V_{\text {bos }}(x)$ (red dashed) vs. quantum modified potential (black solid) $V(x)$ for the periodic potential. The quantum modified potential (1.12) is obtained upon quantizing the fermions and projecting to a fermion number (or spin) eigenstate.

It is well known that there are instanton solutions for the untilted bosonic potentials, with $p=0$. These are real solutions to Newton's equation in the inverted potential, $-V(x)$, given by solutions to the first-order equation (1.3). By connecting degenerate harmonic vacua, this results in the familiar non-perturbative level splitting [5-10]. Instantons are associated with the separatrix (see [50]) in the classical phase space of the inverted $p=0$ potential.

For $p \neq 0$, the standard lore of semi-classics instructs us to search for finite action classical solutions of the equations of motion for the Euclidean action, which correspond to classical motions of a particle in the inverted potential. But this leads to two immediate problems:

- Puzzle 1: For $N_{f}=1$ SUSY QM with a bosonic tilted double-well potential, it is well-known that the perturbatively vanishing ground state energy is lifted nonperturbatively, and supersymmetry is spontaneously broken [48, 49]. However, there is no real classical finite action solution contributing to the ground state energy.

- Puzzle 2: For $N_{f}=1$ SUSY QM with a bosonic Sine-Gordon potential, it is known that the perturbatively vanishing ground state energy remains zero nonperturbatively [48]. However, there is only one real finite action solution which tunnels from one global maximum of the inverted potential to the next. We call this solution the real bion solution because its profile looks like two consecutive 


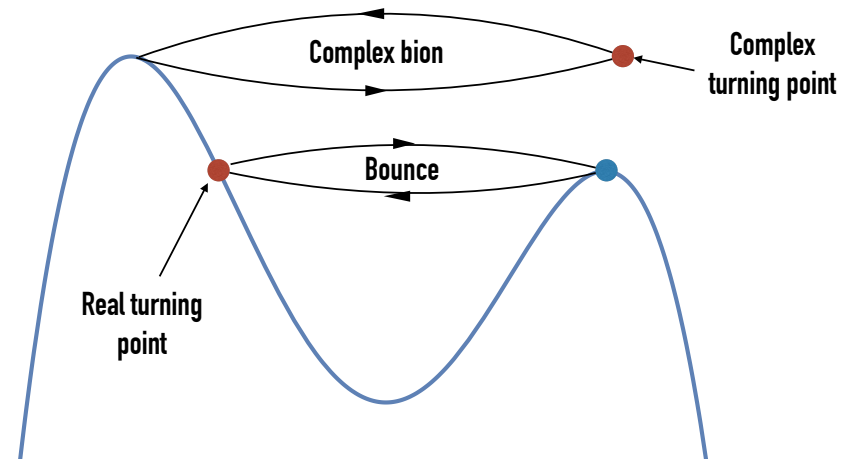

Figure 3. Inverted quantum modified potential in a theory with a symmetric bosonic doublewell potential. There are no real finite action saddle configurations contributing to ground state properties, but there are exact complex saddle solutions. The Euclidean description of the vacuum is a dilute gas of these complex saddle points, which we call complex bions. The complex bion is necessary in order to explain the non-perturbatively induced vacuum energy of the corresponding $N_{f}=1$ SUSY QM model.

instantons. This solution makes a negative contribution to the ground state energy. As it stands, semi-classics would be in conflict with the SUSY algebra, and positive-definiteness of the SUSY Hamiltonian.

We will show that the semi-classical configuration responsible for SUSY breaking in the former case is a complex bion solution which tunnels from the global maximum of the inverted potential to one of two complex turning points, as shown in Figure 3. Moreover, the contribution to ground state energy is $-e^{-S_{\mathrm{cb}}}=-e^{-2 S_{I} \pm i \pi}$, admitting an interpretation in the two-instanton sector of the formulation with fermions, and the $\pm i \pi$ imaginary part of the complex bion action, referred to as the hidden topological angle (HTA) in [26], is crucial for the positive definiteness of the non-perturbative vacuum energy. In the absence of $\pm i \pi$, one would end up with a negative ground state energy, and semi-classics would be in conflict with the constraints of the supersymmetry algebra [49].

In the Sine-Gordon case we will show that in addition to the real bion solution there is a second solution, the complex bion, which tunnels from one global maximum of the inverted potential to one of two complex turning points, as shown in Figure 


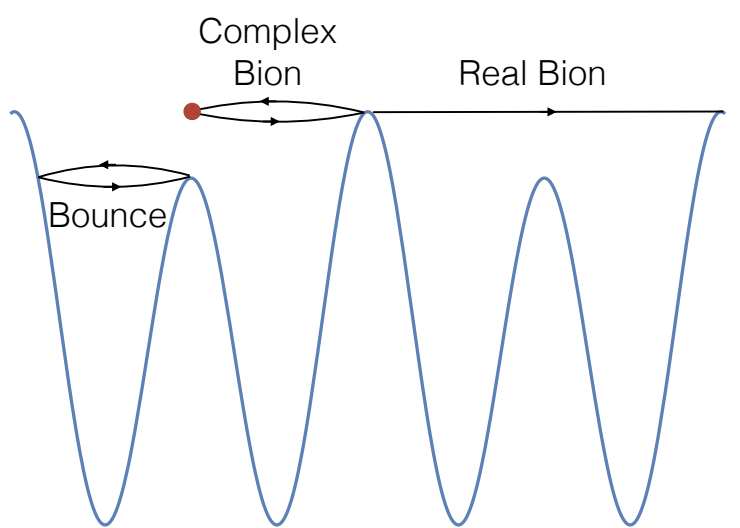

Figure 4. Inverted quantum modified potential in a theory with a periodic bosonic potential. There are exact real and complex saddle solutions in the quantum modified Sine-Gordon model. Both the real and complex bions are necessary in order to explain the non-perturbative vanishing of the vacuum energy of the corresponding $N_{f}=1$ SUSY QM model. The Euclidean description of the vacuum is a dilute gas of real and complex bions.

4. Moreover, the real part of the action is exactly equal to the real bion action, and roughly equal to the twice the instanton action $S_{I}$. The net contribution to the ground state energy is $-e^{-S_{\mathrm{rb}}}-e^{-S_{\mathrm{cb}}}=-e^{-2 S_{I}}-e^{-2 S_{I} \pm i \pi}=0$. Notice that the imaginary part of the complex bion action or HTA, $\pm i \pi$, is necessary for the non-perturbative vanishing of the vacuum energy [26]. One dramatic aspect in this case is that the complex bion solution is both multi-valued, as well as singular, but has finite action with smooth real part. Similar multi-valued solutions also appeared in Ref.[51], which we discuss in Section 8.1.) Since the hidden topological angle is defined modulo $2 \pi$, the naively multi-valued action is in fact single valued, and no pathologies arise for the supersymmetric theory. For non-supersymmetric theories for which the complex bion term comes out as $e^{ \pm i p \pi}$, the semiclassical analysis involves both the HTA and resurgent cancellations.

There is a particularly simple way to obtain the complex bion solutions that we will employ in this work, and one can check that the resulting solutions satisfy the holomorphic Newton equations. In Figs. 3 and 4, there is an obvious exact bounce solution. Starting with the bounce solution, consider an analytic continuation in $p$

$$
p \in \mathbb{R} \rightarrow p e^{i \theta} \in \mathbb{C} .
$$

As $\theta$ changes, the path integral moves into an unphysical region of parameter space, similar to the discussion of [51], where the theory ceases to have a physical Hilbert 
space interpretation. But the end-points of this unphysical region, $\theta=0$ and $\theta=\pi$, correspond to $V_{+}(\mathfrak{x})$ and $V_{-}(\mathfrak{x})$, which are physical theories. However, in the cases studied in this work, the two potentials are related either by parity (mirror images of one another) or a simple shift, and the path integral representation and the set of non-perturbative saddle points associated with them are identical.

Turning on $\theta$ gives the analytic continuation of the bounce into a complex saddle. These complex saddles are plotted in Fig. 14 and Fig. 23. The continuation to $\theta=\pi$ results in complex smooth saddles for the tilted DW case and complex singular saddle for the double-SG example. One interesting aspect of the analytic continuation for the DW system is that one can show that the monodromies associated with the solutions are non-trivial. In fact, as $p$ changes its phase by $2 \pi$, the potential $V_{+}(\mathfrak{x})$ turns back to itself, but the two complex bion solutions are interchanged. Thus, the solutions has a monodromy of order 2, reflecting the two-fold ambiguity in the choice of the exact solutions.

\subsection{What is surprising (and what is not)?}

The necessity of complexification is not surprising from the point of view of the steepest descent method for ordinary integration. Since the path integral is a particular form of infinitely many ordinary integrals, complexification is in fact a natural step. What is interesting and surprising is the important new effects that appear in functional integrals.

As is well known, complexification is both a necessary and sufficient step to capture a complete steepest descent cycle decomposition for ordinary integration. Let $f(x)$ be a real function, and consider an exponential type integral $I(\hbar)=\int_{-\infty}^{\infty} d x e^{-\frac{1}{\hbar} f(x)}$ which exists for $\hbar>0$. (We will also consider the continuation $\hbar \rightarrow \hbar e^{i \theta}$.) To tackle the integration via the steepest descent method, the first step is to complexify:

$$
(f(x), \mathbb{R}) \longrightarrow(f(z), \Gamma \in \mathbb{C})
$$

Since $\mathbb{C}$ has twice the real dimension of $\mathbb{R}$ the integration is restricted to a certain middle-dimensional cycle $\Gamma$ in $\mathbb{C}$. The standard procedure is:

$$
I(\hbar)=\int_{-\infty}^{\infty} d x e^{-\frac{1}{\hbar} f(x)} \underbrace{\longrightarrow}_{\text {steepest descent method }} \sum_{\sigma} n_{\sigma} \int_{\mathcal{J}_{\sigma}} d z e^{-\frac{1}{\hbar} f(z)}
$$

where $\mathcal{J}_{\sigma}$ is the steepest descent cycle attached to the critical point $z_{\sigma}$ of $f(z)$, i.e., $f^{\prime}\left(z_{\sigma}\right)=0$ and the interval $\int_{[-\infty, \infty]}=\sum_{\sigma} n_{\sigma} \int_{\mathcal{J}_{\sigma}}=\int_{\Gamma}$ is a sum over the homology cycle decomposition of the pair $(f(z), \mathbb{C})$ despite the fact that the original integration is over 
$\mathbb{R}$. $\mathcal{J}_{\sigma}(\theta)$ cycle is found by solving a complex version of the gradient flow equation (also called Picard-Lefschetz equation)

$$
\frac{d z}{d u}=e^{i \theta} \frac{\partial \bar{f}}{\partial \bar{z}}
$$

where $u$ is gradient flow time. For $\hbar>0$, we take $\theta=0$. Note that the vanishing of the right hand side determines the critical point set $z_{\sigma}$ for the one dimensional integral given in (1.15).

If $\hbar$ is analytically continued to $\hbar e^{i \theta}$, complex saddles $z_{\sigma}$ contribute once their multipliers $n_{\sigma} \neq 0$. In generic situations this is the case. However, consider the case where $\hbar>0$, positive and real. Then, the cycle is $\mathbb{R}$ and the decomposition is saturated by the real saddles on $\mathbb{R}$. Namely, despite the fact that complex saddles are present, their multiplier $n_{\sigma}$ is zero. Contributing cycles $\mathcal{J}_{\sigma}$ still live in $\mathbb{C}$, but in the linear combination $\sum_{\sigma} n_{\sigma} \mathcal{J}_{\sigma}$, the segments that move into the complex domain, always backtrack by some other contribution $\mathcal{J}_{\sigma^{\prime}}$, so the full cycle is just $\mathbb{R}$. In other words, complex saddles in this case do not contribute to real integration.

Consider now a real Euclidean path integral. Based on the fact that the partition function is real, and guided by the arguments given for ordinary integrals above, complex saddles (which would typically give complex contributions) were usually deemed irrelevant, and did not receive sufficient attention. There are two potential ways around the problem that the partition functions must be real and, interestingly, path integrals take advantage of both:

- If the action of a complex saddle point is $S_{\mathrm{r}}+i \pi(\bmod 2 \pi i)$, the reality of the partition function will be preserved. Indeed, path integrals of many supersymmetric theories are of this type.

- If the action of a complex saddle point is $S_{\mathrm{r}}+i p \pi$, where $p \in \mathbb{R}$ arbitrary, this will (naively) render the partition function complex. But this issue is resolved via resurgence, and renders the trans-series sum over saddles real. An explicit example of this phenomenon is discussed in Section 7.

Both of these mechanisms do not take place in one-dimensional real exponential integrals, as we demonstrate in Appendix B. In this sense, the one dimensional exponential integral cannot be used as a guide on this issue.

In fact, the way that the second item is resolved is interesting. The action of saddles contributing to the path integral is often multi-valued (even for a supersymmetric theory). In particular, the action is of the form $S_{\mathrm{r}} \pm i p \pi$. In the past, multi-valued saddles were viewed as "disturbing", since one is trying to calculate a physical and 
unambiguous quantity. A recent serious deliberation on this issue (which at the end remains undecided) can be found in the recent work [51], which we will comment on in detail in Section 8.1.

The main surprise is the following: In order to get a physical real result for the path integral, multi-valued saddle solutions and multi-valued actions are required. The reason is that Borel resummation of perturbation theory is also generically multi-fold ambiguous, with ambiguities related to the action of the multi-valued saddles. The only possible way that the resulting path integral can be real is by virtue of the exact cancellation between these two types of ambiguities.

\subsection{Holomorphic Newton equations}

The holomorphic classical Euclidean equations (1.5) describe coupled motion of the real and imaginary parts of $z(t)=x(t)+i y(t)$. Expressing the holomorphic potential in terms of real and imaginary parts of the potential, $V(z)=V_{\mathrm{r}}(x, y)+i V_{\mathrm{i}}(x, y)$, the holomorphic Newton equation can be re-expressed as:

$$
\frac{d^{2} z}{d t^{2}}=\frac{\partial V}{\partial z} \quad \text { or equivalently } \quad \begin{aligned}
\frac{d^{2} x}{d t^{2}} & =+\frac{\partial V_{\mathrm{r}}}{\partial x} \\
\frac{d^{2} y}{d t^{2}} & =-\frac{\partial V_{\mathrm{r}}}{\partial y}
\end{aligned}
$$

where the Cauchy-Riemann equations, $\partial_{x} V_{\mathrm{r}}=\partial_{y} V_{\mathrm{i}}$ and $\partial_{y} V_{\mathrm{r}}=-\partial_{x} V_{\mathrm{i}}$, are used to obtain the second form. (1.17) implies that the force in the $x$-direction is $\nabla_{x} V_{\mathrm{r}}(x, y)$, and the force in the $y$-direction is $-\nabla_{y} V_{\mathrm{r}}(x, y)$. In this sense, these are different from the classical motion in the inverted real potential $-V_{\mathrm{r}}(x, y)$ in two space dimensions. This aspect underlies some of the exotic features of our exact solutions.

To make this idea more concrete, as it will provide much intuition for the exact solutions, consider the Euclidean Lagrangian $\mathcal{L}^{E}=\frac{1}{2} \dot{x}^{2}+\frac{1}{2} x^{2}$, and energy conservation $E=\frac{1}{2} \dot{x}^{2}-\frac{1}{2} x^{2}$. Obviously, a particle which starts at rest at a typical point in the close vicinity of $x=0$ will simply roll down. Making the Lagrangian holomorphic, and taking advantage of the Cauchy-Riemann equations, we may write

$$
\mathcal{L}^{E}=\frac{1}{2} \dot{z}^{2}+\frac{1}{2} z^{2}, \quad \operatorname{Re} \mathcal{L}^{E}=\frac{1}{2}\left(\dot{x}^{2}-\dot{y}^{2}\right)+\frac{1}{2}\left(x^{2}-y^{2}\right),
$$

which means $-V_{\mathrm{r}}(x, y)=-x^{2}+y^{2}$. A particle which starts at rest at a typical point in the the close vicinity of $(x, y)=(0,0)$ will roll down in $x$, but will roll up in the $y$ direction! This is counter-intuitive if we just think in terms of potential $-x^{2}+y^{2}$, but it makes sense because of the relative sign in the kinetic term $\dot{x}^{2}-\dot{y}^{2}$. For example, a particle that starts at $(x, y)=(0, y(0))$ will evolve in time as $x(t)=0, y(t)=$ $y(0) \cosh (t)$, rolling up in the $y$-direction to infinity. 
Our goal is to study the path integral corresponding to the holomorphic Lagrangian in (1.4), and find saddles in the system where we analytically continue $p$ as given in (1.13). The reason we do so is that it will be fairly easy to find single valued solutions for general $\theta$, but we will in fact observe non-trivial effects exactly at the physical theories $\theta=0, \pi$. The saddle point contributing to ground state properties is in general complex, sometimes even multi-valued and singular, i.e, either the $\theta=0$ or $\theta=\pi$ direction may correspond to a branch-cut, and we will obtain solutions in the cut-plane. The multivalued solutions are either related to resurgent cancellations or to a hidden topological angle. Turning on $\theta$ provides a useful regularization of the singular solutions.

The coupled Euclidean equations of motion for the tilted-double-well system are

$$
\begin{aligned}
& \frac{d^{2} x}{d t^{2}}=+2 x^{3}-6 x y^{2}-2 a^{2} x+p g \cos \theta, \\
& \frac{d^{2} y}{d t^{2}}=-2 y^{3}+6 x^{2} y-2 a^{2} y+p g \sin \theta,
\end{aligned}
$$

and for the double-Sine-Gordon system they are given by

$$
\begin{aligned}
& \frac{d^{2} x}{d t^{2}}=a^{3} \cosh \left(\frac{y}{a}\right) \sin \left(\frac{x}{a}\right)+\frac{p g}{4}\left(\cos \theta \cosh \left(\frac{y}{2 a}\right) \sin \left(\frac{x}{2 a}\right)-\sin \theta \cos \left(\frac{x}{2 a}\right) \sinh \left(\frac{y}{2 a}\right)\right) \\
& \frac{d^{2} y}{d t^{2}}=a^{3} \cos \left(\frac{x}{a}\right) \sinh \left(\frac{y}{a}\right)+\frac{p g}{4}\left(\sin \theta \cosh \left(\frac{y}{2 a}\right) \sin \left(\frac{x}{2 a}\right)+\cos \theta \cos \left(\frac{x}{2 a}\right) \sinh \left(\frac{y}{2 a}\right)\right) .
\end{aligned}
$$

Note how these differ from the corresponding real Newton equations even at $\theta=0$ or $\pi$, at which (1.19) and (1.20) still remain a coupled set of equations, while the equations in the space of real paths are given by

$$
\begin{aligned}
& \frac{d^{2} x}{d t^{2}}=+2 x^{3}-2 a^{2} x+p g \\
& \frac{d^{2} x}{d t^{2}}=a^{3} \sin \left(\frac{x}{a}\right)+\frac{p g}{4} \sin \left(\frac{x}{2 a}\right) .
\end{aligned}
$$

In terms of real paths, (1.21) and (1.22) do not have finite action non-trivial solutions which start arbitrarily close to the global maximum of the inverted potential. For example, for the tilted-double-well case, any real solution starting at the global maximum of the inverted tilted-double-well potential will go off to infinity and have infinite action. For the periodic double-Sine-Gordon potential, there is a real solution connecting neighboring global maxima of the inverted potential; this is the real bion solution.

On the other hand, (1.19) and (1.20) also admit complex finite action solutions, with non-vanishing real and imaginary parts. We will see that these exact solutions 
are crucial for the semiclassical analysis. The existence of the complex bion solutions follows from consideration of the energy conservation equation for a particle starting at the global maximum of the inverted potential of Figure 3 and Figure 4:

$$
\frac{1}{2} \dot{z}^{2}-V(z)=E \text {. }
$$

The turning point equation $V(z)=-E$ has 4 complex solutions. This is clear for the tilted double well, which is described by a fourth order polynomial, and is also true for the double-Sine-Gordon, which can be described by a fourth order polynomial after a suitable change of variables. Two of the turning points are degenerate roots at the global maximum of the inverted potential, while the other points form a complex conjugate pair near the local maximum of the inverted potential (the point corresponding to the false vacuum).

There indeed exist exact complex solutions ${ }^{3}$ for which a particle follows a complex trajectory that reaches one of the complex turning points and backtracks to the initial position. We call such solutions complex bions, shown in Figure 13 for the double-well system. Its real part is reminiscent of an instanton-anti-instanton $[\mathcal{I} \overline{\mathcal{I}}]$ configuration, and in fact $[\mathcal{I} \overline{\mathcal{I}}]$ superposition at a particular complex separation (see Section 4 for details) is a systematic approximation to the exact complex bion solution.

Normally, an $\mathcal{O}(g)$ effect to the bosonic potential would be negligible with no dramatic effects. However, whenever $V_{\text {bos }}(z)$ has degenerate harmonic minima, (this is the class of examples we consider), the instanton is described as the separatrix of the inverted-potential. The "small" term coming from integrating out fermions and projecting onto a definite sector of the Fock space leads to a perturbative splitting of the separatrix by an $\mathcal{O}(g)$ amount. This has dramatic effects as it causes the instanton to cease being an exact solution. A single instanton has infinite action compared to the vacuum, as one of its ends is in the true vacuum, while the other is in the false vacuum. However what used to be an instanton-anti-instanton configuration, for a specific value of a finite complex separation, becomes an approximation to an exact solution. This exact solution is the complex bion.

\subsection{Relation to complex gradient flow and Picard-Lefschetz theory}

Attached to each critical point there exists a cycle $\mathcal{J}_{\sigma}$, as in $(1.15)$, which can be found by solving a complex gradient flow equation, or the Picard-Lefschetz theory, to describe

\footnotetext{
${ }^{3}$ The approximate form of the exact complex bion solutions was noted long ago by Balitsky and Yung [52] in the case of tilted double well potential, see Section 8.
} 
the Lefschetz-thimbles. ${ }^{4}$ In a theory with a field $z(t)$ and action functional $\mathcal{S}(z)$, this amounts to

$$
\begin{aligned}
& \frac{\partial z(t, u)}{\partial u}=+\frac{\delta \overline{\mathcal{S}}}{\delta \bar{z}}=+\left(\frac{d^{2} \bar{z}}{d t^{2}}-\frac{\partial \bar{V}}{\partial \bar{z}}\right) \\
& \frac{\partial \bar{z}(t, u)}{\partial u}=+\frac{\delta \mathcal{S}}{\delta z}=+\left(\frac{d^{2} z}{d t^{2}}-\frac{\partial V}{\partial z}\right)
\end{aligned}
$$

where $u$ is the gradient flow time. Note that vanishing of the right hand side of the gradient system (1.24) is the complexification of the equations of motion, (the holomorphic Newton equations), as it should be. The limit $u=-\infty$ is the critical point of the action, for which the right hand side is zero and gives just the complexified equations of motions (1.17).

The partition function (1.4) can also be expressed as

$$
Z=\int_{\Gamma} D(x+i y) e^{-\frac{1}{\hbar}(\operatorname{Re} \mathcal{S}+i \operatorname{Im} \mathcal{S})}=\int_{\Gamma} D(x+i y) e^{-\frac{1}{\hbar} \int \frac{1}{2}\left(\dot{x}^{2}-\dot{y}^{2}\right)+i \dot{x} \dot{y}+\ldots} .
$$

The "kinetic term" of $y(t)$ is not bounded from below, and one may worry about the convergence of the path integral. However, this is an illusion. The thimble construction guarantees that the integral is convergent. It is an easy exercise to prove, for example for the simple harmonic oscillator, that the middle-dimensional cycle $\Gamma$ reduces to the standard path integral.

Functional Cauchy-Riemann equation: In terms of real and imaginary parts of the complexified field $z(t)=x(t)+i y(t)$, the action satisfies a functional (infinite dimensional) version of the Cauchy-Riemann equation:

$$
\begin{aligned}
\text { Cauchy }- \text { Riemann }: \quad \frac{\delta \mathcal{S}}{\delta \bar{z}}=0, \quad \Longleftrightarrow \quad \frac{\delta \operatorname{Re} \mathcal{S}}{\delta x}=+\frac{\delta \operatorname{Im} \mathcal{S}}{\delta y} \\
\frac{\delta \operatorname{Re} \mathcal{S}}{\delta y}=-\frac{\delta \operatorname{Im} \mathcal{S}}{\delta x}
\end{aligned}
$$

which means that action is a holomorphic functional of $x(t)+i y(t)$. Now, it is also worthwhile to rewrite the complexified equations of motions:

$$
\begin{aligned}
\text { Equation of motion : } \quad \frac{\delta \mathcal{S}}{\delta z}=0, \quad \Longleftrightarrow \quad \frac{\delta \operatorname{Re} \mathcal{S}}{\delta x}=-\frac{\delta \operatorname{Im} \mathcal{S}}{\delta y}, \\
\frac{\delta \operatorname{Re} \mathcal{S}}{\delta y}=+\frac{\delta \operatorname{Im} \mathcal{S}}{\delta x} .
\end{aligned}
$$

\footnotetext{
${ }^{4}$ Various applications of the Lefschetz thimbles to simple systems, and QFT at finite density can be found in [25, 44, 53-58]. Complex saddles appear naturally in QCD at finite chemical potential, e.g, [59, 60], and in real time Feynman path integrals [61, 62].
} 
The combination of (1.27) with the holomorphy condition (1.26) has a simple consequence. The critical points (saddles) of the real part of a holomorphic functional, $\operatorname{Re} \mathcal{S}$, which may be viewed as a Morse functional, are the same as the critical points of $\mathcal{S}$. In particular, using (1.27) and (1.26), the complexified equations of motion can be re-written as

$$
\frac{\delta \operatorname{Re} \mathcal{S}}{\delta x}=0, \quad \frac{\delta \operatorname{Re} \mathcal{S}}{\delta y}=0 .
$$

Consider the saddle $z_{\sigma}(t)$ and the Lefschetz thimble $\mathcal{J}_{\sigma}$ attached to it. The imaginary part of the action remains invariant under the gradient flow time on the thimble. Using (1.24) and the chain rule,

$$
\frac{\partial \operatorname{Im}[\mathcal{S}]}{\partial u}=0
$$

meaning that

$$
\operatorname{Im}[\mathcal{S}(z)]=\operatorname{Im}\left[\mathcal{S}\left(z_{\sigma}\right)\right]
$$

is invariant under the flow.

Given a set of saddles in a quantum mechanical path integral, in the weak coupling regime, there is always a representation where the Euclidean vacuum of the theory may be viewed as a proliferation of these configurations, corresponding to a "dilute gas". Since $\operatorname{Im}\left[\mathcal{S}\left(z_{\sigma}\right)\right]$ is an invariant associated with these saddles and thimbles, it will crucially enter into the Euclidean vacuum description, and play important physical roles. This is the origin of the hidden topological angle (HTA) discussed in [26]. We will provide concrete examples of HTAs. An interesting observation is that the HTA associated with a critical saddle field (1.30) is identical to the one obtained by studying the much simpler QZM-thimble (4.4).

We may consider the real part of the complexified action as a Morse functional, or more precisely a Morse-Bott functional, $h=\operatorname{Re} \mathcal{S}$ which obeys

$$
\frac{\partial \operatorname{Re}[-\mathcal{S}]}{\partial u} \leq 0
$$

indicating downward flow nature of the flow. A Morse function on a manifold $\mathcal{M}$ is a function which has no degenerate critical points on the manifold. Technically the real part of an action with any kind of symmetry is not a Morse function, because there are degeneracies associated with the symmetry of the system, and therefore there is a critical manifold, i.e. a manifold of critical points related by a symmetry. If the Hessian of all directions orthogonal to the critical manifold is non-singular, then such a function is referred to as a Morse-Bott function. The critical points of the real part 
of a holomorphic functional $h=\operatorname{Re} \mathcal{S}$ are the same as the critical points of $\mathcal{S}$, found by solving (1.28). In this paper we study these problems in two prototypical examples, and make these ideas more concrete.

\section{Graded Hilbert spaces}

Outline: In this section, we generalize Witten's construction of graded Hilbert spaces for supersymmetric quantum mechanics [49] $\left(N_{f}=1\right.$ or spin- $\frac{1}{2}$ particle) to multi-flavor non-supersymmetric theories $\left(N_{f}>1\right.$ or internal spin $\left(\frac{1}{2}\right)^{N_{f}}$ particle.) This provides a calculable version of the path integral based on a graded representation for the fermionic determinant. The graded system provides the staring point for obtaining exact non-BPS solutions. The second (and more important) step is the idea of complexification.

\subsection{Supersymmetric QM and its non-supersymmetric generalization}

Consider the Minkowski space action of supersymmetric quantum mechanics with superpotential $\mathcal{W}(\mathfrak{x})[49]$ in canonical normalization: ${ }^{5}$

$$
S_{M}=\int d t\left(\frac{1}{2} \dot{\mathfrak{x}}^{2}-\frac{1}{2}\left(\mathcal{W}^{\prime}\right)^{2}+\frac{1}{2} i(\bar{\psi} \dot{\psi}-\dot{\bar{\psi}} \psi)+\frac{1}{2} \mathcal{W}^{\prime \prime}[\bar{\psi}, \psi]\right)
$$

We will consider a manifestly non-supersymmetric multi-flavor generalization of this class of quantum systems. We introduce $N_{f}$ flavors, coupled as

$$
S_{M}=\int d t\left(\frac{1}{2} \dot{\mathfrak{x}}^{2}-\frac{1}{2}\left(\mathcal{W}^{\prime}\right)^{2}+\frac{1}{2} i\left(\bar{\psi}_{i} \dot{\psi}_{i}-\dot{\bar{\psi}}_{i} \psi_{i}\right)+\frac{1}{2} \mathcal{W}^{\prime \prime}\left[\bar{\psi}_{i}, \psi_{i}\right]\right), \quad i=1, \ldots, N_{f}
$$

For the non-supersymmetric theory, we refer to $\mathcal{W}(\mathfrak{x})$ as the "auxiliary potential". It plays a role similar to the superpotential in the $N_{f}=1$ theory. The study of $N_{f}=1$ case is old, and $N_{f} \geq 2$ generalization and its study is new. ${ }^{6}$

${ }^{5}$ Canonical vs. non-perturbative normalization: We use $\mathfrak{x}$ to denote the bosonic coordinate and $\mathcal{W}$ to denote auxiliary potential (superpotential) with a canonical normalization, $\frac{1}{2} \mathfrak{\mathfrak { r }}(t)^{2}+\frac{1}{2}\left(\mathcal{W}^{\prime}\right)^{2}+$ ... For non-perturbative normalization, we use $x(t)$ and $W(x)$, and Lagrangian reads $\frac{1}{g}\left[\frac{1}{2} \dot{x}(t)^{2}+\right.$ $\left.\frac{1}{2}\left(W^{\prime}\right)^{2}+\ldots\right]$ where $W(x)$ is $g$ independent. The relation between the two is

$$
x(t)=\sqrt{g} \mathfrak{x}(t), \quad \mathcal{W}(\mathfrak{x})=\frac{1}{g} W(\sqrt{g} \mathfrak{x}(t)) .
$$

The latter is more suitable for the non-perturbative treatment of instantons, and the determination of other non-self-dual saddle points.

${ }^{6}$ This type of generalization is motivated by the quantum field theory studies of QCD(adj) and $\mathcal{N}=1$ SYM on $\mathbb{R}^{3} \times S^{1}$, where the former is the multi-flavor non-supersymmetric generalization of the latter, and despite the absence of supersymmetry, it admits a semi-classical calculable regime [1]. 
We can canonically quantize this Lagrangian. Introduce bosonic position $\widehat{\mathfrak{x}}$ and momentum $\widehat{\mathfrak{p}}$ operators obeying canonical commutation relation, as well as fermionic creation $\widehat{\psi}_{+}^{i}$ and annihilation $\widehat{\psi}_{-}$operators obeying anti-commutation relations:

$$
\begin{aligned}
& {[\widehat{\mathfrak{p}}, \widehat{\mathfrak{x}}]=-i, \quad[\widehat{\mathfrak{p}}, \widehat{\mathfrak{p}}]=[\widehat{\mathfrak{x}}, \widehat{\mathfrak{x}}]=0,} \\
& \left\{\widehat{\psi}_{+}^{i}, \widehat{\psi}_{-}^{j}\right\}=\delta^{i j}, \quad\left\{\widehat{\psi}_{+}^{i}, \widehat{\psi}_{+}^{j}\right\}=\left\{\widehat{\psi}_{-}^{i}, \widehat{\psi}_{-}^{j}\right\}=0 .
\end{aligned}
$$

The Hamiltonian of the multi-flavor theory reads

$$
\begin{aligned}
\widehat{H} & =\underbrace{\frac{1}{2} \widehat{\mathfrak{p}}^{2}+\frac{1}{2}\left(\mathcal{W}^{\prime}\right)^{2}}_{\widehat{H}^{b}}+\frac{1}{2}\left[\widehat{\psi}_{+}^{i}, \widehat{\psi}_{-}^{i}\right] \mathcal{W}^{\prime \prime} \\
& =\widehat{H}^{b}+\left(\widehat{N}-\frac{1}{2} N_{f}\right) \mathcal{W}^{\prime \prime},
\end{aligned}
$$

where summation over repeated indices is assumed. In bringing the Hamiltonian into the latter form, we used $\sum_{i=1}^{N_{f}}\left[\widehat{\psi}_{+}^{i}, \widehat{\psi}_{-}^{i}\right]=\sum_{i=1}^{N_{f}}\left(2 \widehat{\psi}_{+}^{i} \widehat{\psi}_{-}^{i}-1\right) \equiv 2 \widehat{N}-N_{f}$, where $\widehat{N}$ is the "fermion number" (or equivalently spin) operator. $\widehat{H}^{b}$ denotes the Hamiltonian of the $N_{f}=0$ (purely bosonic) theory.

The Lagrangian is invariant under a $U(1) \times S U\left(N_{f}\right)$ global flavor symmetry:

$$
\psi_{i} \rightarrow e^{i \alpha} U_{i j} \psi_{j}, \quad e^{i \alpha} \in U(1), \quad U \in S U\left(N_{f}\right) .
$$

This means that the Hilbert space is decomposable into irreducible representations of $U(1) \times S U\left(N_{f}\right)$. The charges associated with these global symmetries are

$$
\widehat{N}=\sum_{i=1}^{N_{f}} \widehat{\psi}_{+}^{i} \widehat{\psi}_{-}^{i}, \quad \widehat{S}^{a}=\widehat{\psi}_{+}^{i}\left(t^{a}\right)_{i j} \widehat{\psi}_{-}^{j},
$$

where $t^{a}, a=1, \ldots N_{f}^{2}-1$ are the generators of the defining representation of the $\mathfrak{s u}\left(N_{f}\right)$ algebra. Clearly, these generators commute with the Hamiltonian,

$$
[\widehat{N}, \widehat{H}]=0, \quad\left[\widehat{S}^{a}, \widehat{H}\right]=0 .
$$

$\widehat{N}$ allow us to express the full Hilbert space as a direct sum of graded Hilbert spaces according to fermion number, and graded Hilbert spaces naturally fall into irreducible anti-symmetric representation of the $S U\left(N_{f}\right)$ global symmetry.

\section{$2.2 \quad$ Fermionic Fock space}

The fermionic operators can be used to build a finite dimensional fermionic Hilbert space $\mathcal{F}$. Let $|\Omega\rangle$ denote the Fock vacuum, singlet under the $S U\left(N_{f}\right)$ flavor symmetry. It obeys

$$
\widehat{\psi}_{-}^{i}|\Omega\rangle=0 \quad \forall i \in\left[1, N_{f}\right]
$$


There is one more singlet under the $S U\left(N_{f}\right)$ flavor symmetry. It is the fully occupied state, defined as

$$
|\widetilde{\Omega}\rangle=\widehat{\psi}_{+}^{1} \widehat{\psi}_{+}^{2} \ldots \widehat{\psi}_{+}^{N_{f}}|\Omega\rangle
$$

and by the Pauli exclusion principle, it obeys

$$
\widehat{\psi}_{+}^{i}|\widetilde{\Omega}\rangle=0 \quad \forall i \in\left[1, N_{f}\right] .
$$

We can now construct the fermionic Hilbert space, which furnishes $k$-index anti-symmetric irreps of $S U\left(N_{f}\right)$, where $k=0, \ldots, N_{f}$ denotes the fermion number sector, where $k$ is eigenvalue of the fermion number operator $\widehat{N}$. There are $N_{f}+1$ levels, and $2^{N_{f}}$ states:

$$
\mathcal{F}=\bigoplus_{k=0}^{N_{f}} \mathcal{F}^{(k)}
$$

where

$$
\begin{aligned}
& \mathcal{F}^{(0)}:=\{|\Omega\rangle\}, \\
& \mathcal{F}^{(1)}:=\left\{\widehat{\psi}_{+}^{i}|\Omega\rangle\right\}, \\
& \mathcal{F}^{(2)}:=\left\{\widehat{\psi}_{+}^{i} \widehat{\psi}_{+}^{j}|\Omega\rangle\right\}, \\
& \vdots \\
& \mathcal{F}^{\left(N_{f}-2\right)}:=\left\{\widehat{\psi}_{-}^{i} \widehat{\psi}_{-}^{j}|\widetilde{\Omega}\rangle\right\} \\
& \mathcal{F}^{\left(N_{f}-1\right)}:=\left\{\widehat{\psi}_{-}^{i}|\widetilde{\Omega}\rangle\right\}, \\
& \mathcal{F}^{\left(N_{f}\right)}:=\{|\widetilde{\Omega}\rangle\}
\end{aligned}
$$

with degeneracies at level $k$ given by $\operatorname{deg}_{k}=\left(\begin{array}{c}N_{f} \\ k\end{array}\right)$ given by the dimension of $k$-index anti-symmetric irreps of $S U\left(N_{f}\right)$. The total number of states is then

$$
\sum_{k=0}^{N_{f}} \operatorname{deg}_{k}=\sum_{k=0}^{N_{f}}\left(\begin{array}{c}
N_{f} \\
k
\end{array}\right)=1+N_{f}+\frac{N_{f}\left(N_{f}-1\right)}{2}+\ldots+N_{f}+1=2^{N_{f}} .
$$

Both the unoccupied state and the fully occupied state are singlets under $S U\left(N_{f}\right)$. Since the ground states of quantum mechanics must be singlets under bosonic global symmetries, we should expect the $|\Omega\rangle$ and $|\widetilde{\Omega}\rangle$ states to play significant roles in the ground state properties. 


\subsection{Graded Hilbert space: Fermion number representation}

The Hamiltonian of the multi-flavor theory is diagonal in the fermion number operator. This means that the decomposition of the fermionic Fock space translates in a simple way to the decomposition of the full Hilbert space. Any eigenstate of the full Hamiltonian can be written as $|n\rangle_{B} \otimes|\chi\rangle_{F}$, where $|\chi\rangle_{F} \in \mathcal{F}$. If $|\chi\rangle_{F} \in \mathcal{F}^{(k)}$, then, $\left(2 \widehat{N}-N_{f}\right)|\chi\rangle=\left(2 k-N_{f}\right)|\chi\rangle$, and the action of the level $k$ Hamiltonian on the state $|n\rangle_{B}$ is

$$
\widehat{H}_{\left(N_{f}, k\right)}=\frac{\mathfrak{p}^{2}}{2}+\frac{1}{2}\left(\mathcal{W}^{\prime}(\mathfrak{x})\right)^{2}+\frac{p}{2} \mathcal{W}^{\prime \prime}(\mathfrak{x}), \quad p=2 k-N_{f}, \quad k=0, \ldots, N_{f} .
$$

This permits us to decompose the Hilbert space $\mathcal{H}$ of $N_{f}$ flavor theory into sub-Hilbert spaces $\mathcal{H}_{\left(N_{f}, k\right)}$ graded under the "fermion number" $k$. The Hamiltonian $\widehat{H}$ and the Hilbert space $\mathcal{H}$ of the $N_{f}$ flavor theory can be decomposed as:

$$
\begin{aligned}
\widehat{H} & =\bigoplus_{k=0}^{N_{f}} \operatorname{deg}\left(\mathcal{H}_{\left(N_{f}, k\right)}\right) \widehat{H}_{\left(N_{f}, k\right)}, \quad \operatorname{deg}\left(\mathcal{H}_{\left(N_{f}, k\right)}\right)=\left(\begin{array}{c}
N_{f} \\
k
\end{array}\right) \\
\mathcal{H} & =\bigoplus_{k=0}^{N_{f}} \operatorname{deg}\left(\mathcal{H}_{\left(N_{f}, k\right)}\right) \mathcal{H}_{\left(N_{f}, k\right)},
\end{aligned}
$$

where $\operatorname{deg}\left(\mathcal{H}_{\left(N_{f}, k\right)}\right)$ is the degeneracy of a given sector. This construction generalizes Witten's discussion in supersymmetric QM to non-supersymmetric theories [48]. We obtain a triangle of graded Hilbert spaces, where black ( $k$-even) are bosonic, and red $(k$-odd) are fermionic spaces:

$$
\begin{aligned}
& 1 \mathcal{H}_{(0,0)} \\
& 1 \mathcal{H}_{(1,0)} \quad 1 \mathcal{H}_{(1,1)} \\
& 1 \mathcal{H}_{(2,0)} \quad 2 \mathcal{H}_{(2,1)} \quad 1 \mathcal{H}_{(2,2)} \\
& \begin{array}{llll}
\mathcal{H}_{(3,0)} & 3 \mathcal{H}_{(3,1)} & 3 \mathcal{H}_{(3,2)} & 1 \mathcal{H}_{(3,3)}
\end{array} \\
& \begin{array}{lllll}
1 \mathcal{H}_{(4,0)} & 4 \mathcal{H}_{(4,1)} & 6 \mathcal{H}_{(4,2)} & 4 \mathcal{H}_{(4,3)} & 1 \mathcal{H}_{(4,4)}
\end{array}
\end{aligned}
$$

The $\left(N_{f}, k\right)=(0,0)$ cell is the bosonic Hilbert space, $\mathcal{H}_{(0,0)}$. The $N_{f}=1$ row contains the paired Hilbert spaces of supersymmetric quantum mechanics [48]. The study of $N_{f} \geq 2$ is new and has some surprising elements. 
It is also useful to further grade the Hilbert spaces under fermion number modulo two, $(-1)^{F}$. For example, if the state $|0\rangle_{B} \otimes|\Omega\rangle$ is a ground state, then, obviously, $\mathcal{H}_{0}$ is bosonic, $\mathcal{H}_{1}$ is fermionic, $\mathcal{H}_{2}$ is bosonic, etc. If $N_{f}$ is odd, then $\mathcal{H}_{N_{f}}$ is fermionic, while if $N_{f}$ is even, then $\mathcal{H}_{N_{f}}$ is bosonic.

The advantage of the graded formulation is the following. Given the twisted partition function of the theory with multiple fermions, we can now convert it into the sum of bosonic partition functions over the graded sectors:

$$
\begin{aligned}
\tilde{Z}(L) & \equiv \operatorname{Tr}_{\mathcal{H}}(-1)^{F} e^{-L H} \\
& =\sum_{k=0}^{N_{f}}(-1)^{N_{f}-k} \operatorname{deg}\left(\mathcal{H}_{k}\right) \operatorname{Tr}_{\mathcal{H}_{k}} e^{-L H_{k}} \\
& =\sum_{k=0}^{N_{f}}(-1)^{N_{f}-k} \operatorname{deg}\left(\mathcal{H}_{k}\right) Z_{k}
\end{aligned}
$$

\subsection{Derivation from the fermionic determinant}

We can also derive (2.18) by using a functional integral formulation. Consider

$$
\begin{aligned}
\tilde{Z}(L)^{\text {original }} & =\int D \mathfrak{x} \prod_{i=1}^{N_{f}} D \psi_{i} D \psi_{i} e^{-\int d t\left(\frac{1}{2} \mathfrak{\mathfrak { x }}^{2}+\frac{1}{2}\left(\mathcal{W}^{\prime}\right)^{2}+\bar{\psi}_{i}\left(\partial_{t}+\mathcal{W}^{\prime \prime}\right) \psi_{i}\right)} \\
& =\int D \mathfrak{x} e^{-\int d t \mathcal{L}_{\text {bos }}}\left[\operatorname{det}_{ \pm}\left(\partial_{t}+\mathcal{W}^{\prime \prime}\right)\right]^{N_{f}}
\end{aligned}
$$

The fermionic determinant can be calculated exactly [63]. The result for periodic $(+)$ and anti-periodic $(-)$ boundary conditions on the fermions is given by

$$
\left[\operatorname{det}_{ \pm}\left(\partial_{t}+\mathcal{W}^{\prime \prime}\right)\right]^{N_{f}}= \begin{cases}{\left[2 \sinh \left(\frac{1}{2} \int \mathcal{W}^{\prime \prime} d t\right)\right]^{N_{f}}} & \text { for } \psi_{i}(L)=+\psi_{i}(0) \\ {\left[2 \cosh \left(\frac{1}{2} \int \mathcal{W}^{\prime \prime} d t\right)\right]^{N_{f}}} & \text { for } \psi_{i}(\beta)=-\psi_{i}(0)\end{cases}
$$

This implies that we can express the twisted partition function as

$$
\tilde{Z}(L)=\int D \mathfrak{x} e^{-\int d t \mathcal{L}_{\mathrm{bos}}-N_{f} \log \left(2 \sinh \frac{1}{2} \int d t \mathcal{W}^{\prime \prime}\right)} .
$$

This strategy is commonly used in QFT applications of the fermionic determinant. We obtain a closed form result, but it is manifestly non-local ${ }^{7}$. In the present case,

\footnotetext{
${ }^{7}$ Notice the fact that the determinant makes the sign problem manifest if $\mathcal{W}(\mathfrak{x})$ is an odd function of $\mathfrak{x}$. Under $\mathfrak{x}(t) \rightarrow-\mathfrak{x}(t)$, the second term in the action picks up an extra phase $e^{i \pi N_{f}}$, leading to
} 
however, there is a more useful representation. Instead of exponentiating the fermionic determinant, if we just expand it into a binomial expansion, we obtain the link between the fermionic determinant and the graded Hilbert spaces. We find

$$
\begin{aligned}
\tilde{Z}(L)^{\text {original }} & =\int D \mathfrak{x} e^{-\int d t \mathcal{L}_{\mathrm{bos}}}\left[2 \sinh \frac{1}{2} \int d t \mathcal{W}^{\prime \prime}\right]^{N_{f}} \\
& =\int D \mathfrak{x} e^{-\int d t \mathcal{L}_{\mathrm{bos}}} \sum_{k=0}^{N_{f}}\left(\begin{array}{c}
N_{f} \\
k
\end{array}\right)(-1)^{N_{f}-k}\left[e^{\int d t \frac{\mathcal{W}^{\prime \prime}}{2}}\right]^{k}\left[e^{-\int d t \frac{\mathcal{W}^{\prime \prime}}{2}}\right]^{N_{f}-k} \\
& \left.=\sum_{k=0}^{N_{f}}\left(\begin{array}{c}
N_{f} \\
k
\end{array}\right)(-1)^{N_{f}-k} \int D \mathfrak{x} e^{-\int d t\left(\frac{1}{2} \dot{\mathfrak{x}}^{2}+\frac{1}{2}\left(\mathcal{W}^{\prime}\right)^{2}+\left(2 k-N_{f}\right) \frac{\mathcal{W}^{\prime \prime}}{2}\right.}\right) \\
& =\sum_{k=0}^{N_{f}}\left(\begin{array}{c}
N_{f} \\
k
\end{array}\right)(-1)^{N_{f}-k} Z_{k}=Z(L)^{\text {graded }}
\end{aligned}
$$

This simple equality has great utility. In particular, the graded formulation will allow us to find new exact saddles in the problem. In the original formulation where we keep the fermions, it is more difficult to demonstrate the existence of exact solutions. The beauty of the graded formulation is that we can show that these approximate solutions are actually approximations to the exact solutions.

Consider taking the compact radius $L$ to be infinitely large, and restrict to solutions starting and ending in a particular harmonic minimum, which we label by $\mathfrak{x}_{i}$. These are the solutions which are interesting if we wish to quantize around the classical minimum at $\mathfrak{x}=\mathfrak{x}_{i}$. Then $\mathcal{W}^{\prime \prime}(x(t)) \approx \mathcal{W}^{\prime \prime}\left(x_{i}\right)=\omega_{i}$, the natural local harmonic frequency, almost everywhere on this solution. The determinant in the infinite volume limit $L \rightarrow \infty$ will then become a simple exponential

$$
\left[\operatorname{det}_{ \pm}\left(\partial_{t}+\mathcal{W}^{\prime \prime}\right)\right]^{N_{f}} \underbrace{=}_{L \rightarrow \infty} \begin{cases}\exp \left(\frac{\operatorname{sign}\left(\omega_{i}\right) N_{f}}{2} \int \mathcal{W}^{\prime \prime} d t\right) & \text { for } \psi_{i}(L)=+\psi_{i}(0) \\ \left(\operatorname{sign}\left(\omega_{i}\right)\right)^{N_{f}} \exp \left(\frac{\operatorname{sign}\left(\omega_{i}\right) N_{f}}{2} \int \mathcal{W}^{\prime \prime} d t\right) & \text { for } \psi_{i}(\beta)=-\psi_{i}(0) .\end{cases}
$$

The above form yields a perfectly local action which corresponds to the projection to the $k=0$ (empty) or $k=N_{f}$ (fully occupied) fermion number eigenstates. This corresponds to maximal spin projection, i.e. $S_{z}= \pm N_{f} / 2$. The reason this is the case

destructive interference between the Euclidean paths $\mathfrak{x}(t)$ and $-\mathfrak{x}(t)$ for $N_{f}$ odd, and to constructive interference for $N_{f}$ even. This is a manifestation of the sign problem, $\tilde{Z}(L) \sim\left(1+e^{i \pi N_{f}}\right)$ in the odd- $N_{f}$ theory, see for example, [64] for the $N_{f}=1$ case. The sign problem in these examples is related to exact spectral cancellations. 
is that the ground state(s) lives in the maximal spin state, and sending $L \rightarrow \infty$ projects onto the ground-state(s) only.

\subsection{Graded Hilbert space: Spin representation}

For $N_{f}=1$, the full Hamiltonian can be interpreted as describing the motion of a spin$\frac{1}{2}$ particle in a spin-independent potential, $\left(\mathcal{W}^{\prime}\right)^{2}$, and a "magnetic field", $\mathcal{W}^{\prime \prime}$, which couples to the spin. This is the case with exact supersymmetry in the interpretation of Ref. [49]. For $N_{f}=2$, we have a spin $\frac{1}{2} \otimes \frac{1}{2}=1 \oplus 0$ particle, a spin- 1 , and a spin- 0 particle in a magnetic field. For few low- $N_{f}$, we have

$$
\begin{aligned}
& N_{f}=0, \quad 0, \\
& N_{f}=1, \quad \frac{1}{2}, \\
& N_{f}=2, \quad \frac{1}{2} \otimes \frac{1}{2}=1(1) \oplus 1(0), \\
& N_{f}=3, \quad \frac{1}{2} \otimes \frac{1}{2} \otimes \frac{1}{2}=1\left(\frac{3}{2}\right) \oplus 2\left(\frac{1}{2}\right), \\
& N_{f}=4, \quad \frac{1}{2} \otimes \frac{1}{2} \otimes \frac{1}{2} \otimes \frac{1}{2}=1(2)+3(1) \oplus 2(0), \\
& N_{f}=5, \quad \frac{1}{2} \otimes \frac{1}{2} \otimes \frac{1}{2} \otimes \frac{1}{2} \otimes \frac{1}{2}=1\left(\frac{5}{2}\right)+4\left(\frac{3}{2}\right) \oplus 5\left(\frac{1}{2}\right) \text {. }
\end{aligned}
$$

In general, decomposing

$$
\left(\frac{1}{2}\right)^{N_{f}}=\bigoplus_{S=S_{\min }}^{S_{\max }} \operatorname{mult}(S) S
$$

where

$$
S_{\max }=\frac{N_{f}}{2}, \quad S_{\min }= \begin{cases}0 & N_{f} \text { even }, \\ \frac{1}{2} & N_{f} \text { odd }\end{cases}
$$

the multiplicity of the spin- $S$ sector is given by

$$
S=\frac{N_{f}}{2}-k, \quad \operatorname{mult}(S)= \begin{cases}1 & k=0, \\
\left(\begin{array}{c}
N_{f} \\
k
\end{array}\right)-\left(\begin{array}{c}
N_{f} \\
k-1
\end{array}\right) & 1 \leq k \leq\left\lfloor\frac{N_{f}}{2}\right\rfloor .\end{cases}
$$

Therefore, the fermionic Fock space admits a representation both in terms of fermion number sectors as well as spin sectors. It is easy to show that the dimension of the Fock space agree:

$$
2^{N_{f}}=\sum_{k=0}^{N_{f}} \operatorname{deg}\left(\mathcal{H}_{k}\right)=\sum_{S=S_{\min }}^{S_{\max }} \operatorname{mult}(S)(2 S+1)
$$




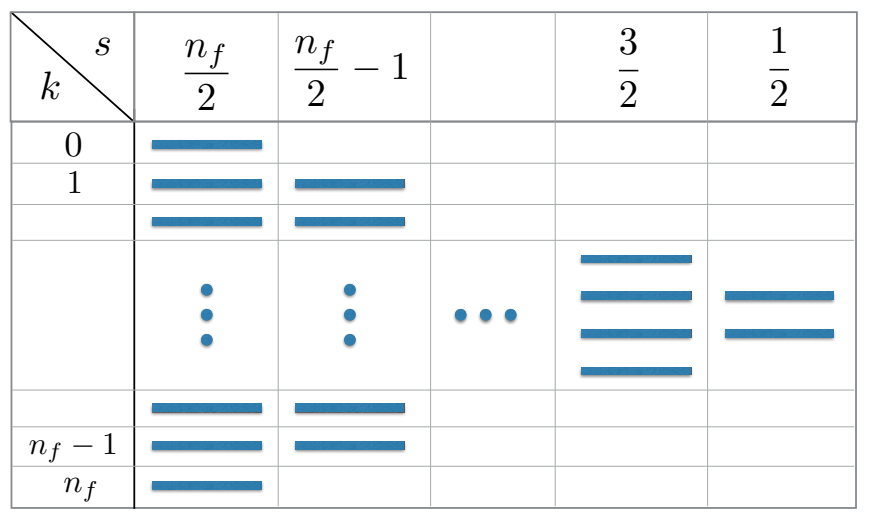

Figure 5. Fermion number vs. spin representation for the multi-flavor theory with Lagrangian (2.3). $\quad N_{f}$ is chosen to be odd. The system describes a particle with internal $\left(\frac{1}{2}\right)^{N_{f} \text {-spin. }}$

One can re-interpret the Hamiltonian of the "multi-flavor" theory as a direct sum over allowed spin quantum numbers, appearing on the right hand side of (2.25):

$$
\widehat{H}=\bigoplus_{S=S_{\min }}^{S_{\max }} \operatorname{mult}(S) \widehat{H}^{(S)} .
$$

The Hamiltonian in the spin- $S$ sector is given by:

$$
\widehat{H}^{(S)}=\frac{1}{2} \widehat{\mathfrak{p}}^{2}+\frac{1}{2}\left(\mathcal{W}^{\prime}\right)^{2}+S_{z} \mathcal{W}^{\prime \prime}, \quad \text { where } S_{z}=\operatorname{Diag}(s, s-1, \ldots,-s+1,-s),
$$

is the diagonal spin matrix. This provides a second representation for the thermal partition function:

$$
Z(\beta) \equiv \sum_{S=S_{\min }}^{S_{\max }} \operatorname{mult}(S) Z_{S},
$$

where $Z_{S}$ is the partition function in the spin- $S$ sector. Note that odd $N_{f}$ is associated with a sum over half-integer spins, and even $N_{f}$ is associated with a sum over integer spins.

The partition function for the $S=\frac{1}{2}$ case (or $N_{f}=1$ supersymmetric QM) is given by (see $[8,9]$ for discussions concerning the path integral representation of spin)

$$
\begin{aligned}
Z(\beta) & =\int D \mathfrak{x} D(\cos \theta) D \phi e^{-\int d t\left(\frac{1}{2} \mathfrak{x}^{2}+\frac{1}{2}\left(\mathcal{W}^{\prime}\right)^{2}+S_{B}(\theta, \phi)+S_{W Z}(\theta, \phi)\right)}, \\
S_{B}(\theta, \phi) & =\int d t \frac{1}{2} \mathcal{W}^{\prime \prime} \cos \theta, \quad S_{W Z}(\theta, \phi)=i \frac{1}{2} \int d t(1-\cos \theta) \partial_{t} \phi,
\end{aligned}
$$


where $(\theta, \phi) \in \mathbf{S}^{2}$ parameterize the Bloch sphere. $S_{B}(\theta, \phi)$ denotes the spin- "magnetic field" interaction, and $S_{W Z}(\theta, \phi)$ is the abelian Berry phase (or Wess-Zumino term). For a spin- $S$ sector, the generalization of this expression is ${ }^{8}$

$$
Z_{S}(\beta)=\int D \mathfrak{x} D(\cos \theta) D \phi e^{-\int d t\left(\frac{1}{2} \dot{\mathfrak{x}}^{2}+\frac{1}{2}\left(\mathcal{W}^{\prime}\right)^{2}+S\left(\mathcal{W}^{\prime \prime} \cos \theta+i(1-\cos \theta) \partial_{t} \phi\right)\right.}
$$

In this work we will not take full advantage of the spin-representation. However, it is useful to keep in mind that the most physically appropriate interpretation of the problem is actually that of a particle with internal spin $\left(\frac{1}{2}\right)^{N_{f}}$. In fact, the equivalence of (2.33) and (2.18) is straightforward. The relation between the partition function graded according to fermion number and spin representations is given by

$$
Z(\beta) \equiv \underbrace{\sum_{k=0}^{N_{f}} \operatorname{deg}\left(\mathcal{H}_{k}\right) Z_{k}}_{\text {sum over columns in Figure } 5}=\underbrace{\sum_{S=S_{\min }}^{S_{\max }} \operatorname{mult}(S) Z_{S}}_{\text {sum over rows in Figure 5 }} .
$$

\subsection{Twisted partition function vs. supersymmetric Witten index}

The twisted partition function (2.18) $\tilde{Z}(L)$ is identical to the supersymmetric Witten index $I_{W}$ [49] for the $N_{f}=1$ theory. For non-supersymmetric theories with $N_{f}>1$ the twisted partition function has a set of remarkable properties, which are still connected to spectral cancellations (under the conditions stated below) in non-supersymmetric theories. In particular, we will show that for certain choices of the auxiliary potential $\mathcal{W}(\mathfrak{x})$, the twisted partition function (2.18) vanishes for any odd $N_{f}$.

First note the following three properties regarding the structure of the graded Hilbert space (2.18):

i) The degeneracies of states in $\mathcal{H}_{k}$ and $\mathcal{H}_{N_{f}-k}$ are the same: $\operatorname{deg}\left(\mathcal{H}_{k}\right)=\operatorname{deg}\left(\mathcal{H}_{N_{f}-k}\right)=$ $\left(\begin{array}{c}N_{f} \\ k\end{array}\right)$ as dictated by the dimensions of corresponding irreps of $S U\left(N_{f}\right)$.

ii) For $N_{f}$ even, $\mathcal{H}_{k}$ and $\mathcal{H}_{N_{f}-k}$ are either Bose-Bose or Fermi-Fermi pairs. For $N_{f}$ odd, $\mathcal{H}_{k}$ and $\mathcal{H}_{N_{f}-k}$ form Bose-Fermi pairs.

iii) If the auxiliary potential is an odd function of $\mathfrak{x}, \mathcal{W}(-\mathfrak{x})=-\mathcal{W}(\mathfrak{x})$, or a periodic function, then $\mathcal{H}_{k}$ and $\mathcal{H}_{N_{f}-k}$ exhibit spectral degeneracy, $\operatorname{spec}\left(\widehat{H}_{k}\right)=$ $\operatorname{spec}\left(\widehat{H}_{N_{f}-k}\right)$.

\footnotetext{
${ }^{8}$ The result we give here is for the thermal partition function. For the twisted partition function an insertion $(-1)^{N_{f}-k}$ needs to be inserted. Since $S_{z}=k-N_{f} / 2$, with $k$ being the fermion number, and since $S_{z}=S \cos \theta$, to implement this twist we can insert a term $i \pi \frac{1}{L} \int_{0}^{L} d t\left(S \cos \theta-N_{f} / 2\right)$ into the action.
} 
If these conditions hold, the twisted partition function for odd- $N_{f}$ theories is "trivial" and vanishes:

$$
\tilde{Z}(L) \equiv \operatorname{Tr}_{\mathcal{H}}(-1)^{F} e^{-L H}=\sum_{k=0}^{N_{f}} \operatorname{deg}\left(\mathcal{H}_{k}\right)(-1)^{N_{f}-k} \operatorname{Tr}_{\mathcal{H}_{k}} e^{-L H_{k}}=0
$$

Readers familiar with supersymmetric quantum mechanics will immediately realize that with the choice of $\mathcal{W}(\mathfrak{x})$ quoted in iii), the Witten index is identically zero, $I_{W}=0$. As a reminder, we note that $I_{W}=0$ means one of two things: a) Supersymmetry is spontaneously broken, and there are degenerate positive energy grounds states. b) Supersymmetry is not broken, and there are degenerate zero energy grounds states. In both cases, regardless of spontaneous breaking of supersymmetry, there is an exact spectral cancellation between bosonic and fermionic Hilbert spaces. Remarkably enough, the spectral cancellation generalizes to multi-flavor non-supersymmetric quantum mechanics for this general class of auxiliary potentials, and we will take advantage of that.

\subsection{Isospectral pairs}

Let $\mathcal{W}(\mathfrak{x})$ be an odd-polynomial, which means $\mathcal{W}^{\prime}(x)$ is even and $\mathcal{W}^{\prime \prime}(x)$ is odd. Consider

$$
\widehat{H}_{k}=\widehat{H}_{\mathrm{bos}}+\left(k-\frac{N_{f}}{2}\right) \mathcal{W}^{\prime \prime}, \quad \widehat{H}_{N_{f}-k}=\widehat{H}_{\mathrm{bos}}-\left(k-\frac{N_{f}}{2}\right) \mathcal{W}^{\prime \prime}
$$

Let $\psi_{k, n}(x)$ be an eigenstate of $\widehat{H}_{k}$ with eigenvalue $E_{k, n}: \widehat{H}_{k} \psi_{k, n}(x)=E_{k, n} \psi_{k, n}(x)$. Acting with parity operator from the left, and realizing simple identity ${ }^{9}$ :

$$
P \widehat{H}_{k} P=\widehat{H}_{N_{f}-k}
$$

we reach to the conclusion that $P \psi_{k, n}(x)=\psi_{k, n}(-x)$ is an eigenstate of $\widehat{H}_{N_{f}-k}$ with the same eigenvalue. Thus, we identify $\psi_{k, n}(-x)=\psi_{N_{f}-k, n}(x)$, and $E_{k, n}=E_{N_{f}-k, n}$. This demonstrates isospectrality for odd-polynomial auxiliary potentials.

For periodic potentials, such as $\mathcal{W}(\mathfrak{x})=\frac{4 a^{3}}{g} \cos \frac{\mathfrak{x} \sqrt{g}}{2 a}$, the demonstration is almost the same, with a minor difference. The potential appearing in $\widehat{H}_{k}$ is of the form $\frac{1}{2}\left(\mathcal{W}^{\prime}\right)^{2}+\left(k-\frac{N_{f}}{2}\right) \mathcal{W}^{\prime \prime}$. This implies that the period of $\left(\mathcal{W}^{\prime}\right)^{2}$ is $\frac{\mathfrak{x} \sqrt{g}}{2 a} \sim \frac{\mathfrak{x} \sqrt{g}}{2 a}+\pi$, while the period of $\mathcal{W}^{\prime \prime}$ is $2 \pi$. Based on this observation

$$
T_{\pi} \widehat{H}_{k} T_{\pi}^{\dagger}=\widehat{H}_{N_{f}-k}
$$

\footnotetext{
${ }^{9}$ This identity fails if $\mathcal{W}(\mathfrak{x})$ is an even-polynomial. In that case, $P \widehat{H}_{k} P=\widehat{H}_{k}$ with no non-trivial implications.
} 

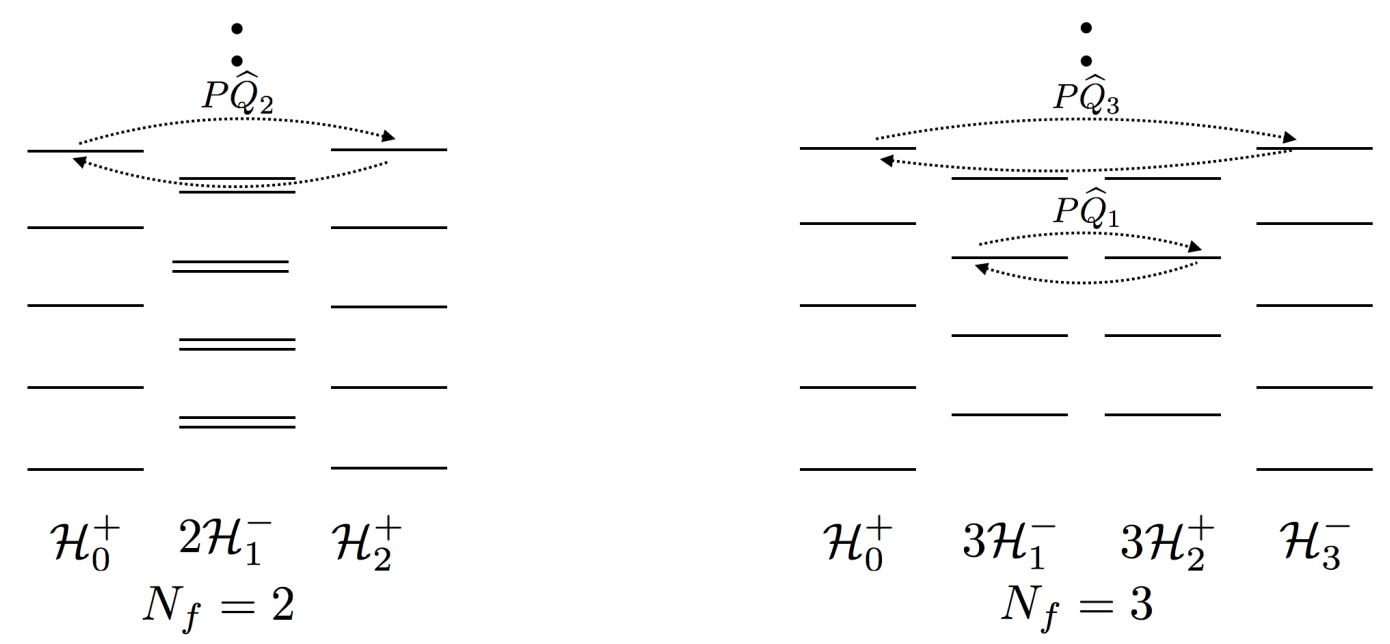

Figure 6. Hilbert-space of the $N_{f}=2$ (prototype for even $N_{f}$ ) and $N_{f}=3$ (prototype for odd $N_{f}$ ) flavor theories with odd auxiliary potential $W(x)$. The Hilbert space decompose according to fermion number. We use the notation $\left(\operatorname{deg}_{k}\right) \mathcal{H}_{k}^{ \pm}$to label a graded Hilbert space, where \pm is the value under $(-1)^{F}$. For odd $N_{f}$, all sectors are paired via nilpotent operators, $P Q$, for even $N_{f}$, all but the middle one are paired. In both cases, there are two ground states.

where $T_{\pi}$ is shift operator by $\pi$. This also implies, for periodic potentials, $\widehat{H}_{k}$ and $\widehat{H}_{N_{f}-k}$ are isospectral.

Although this is sufficient to demonstrate isospectrality, it is also useful to construct this mapping in terms of operators acting in the full Hilbert space $\mathcal{H}$, i.e, restore the fermion operators in the above discussion. To this end, we note that we can define the following classes of nilpotent operators as maps from $\mathcal{F}^{(k)}$ to $\mathcal{F}^{\left(N_{f}-k\right)}$, namely,

$$
\begin{array}{ll}
\widehat{Q}_{N_{f}}=\left\{\widehat{\psi}_{+}^{1} \widehat{\psi}_{+}^{2} \ldots \widehat{\psi}_{+}^{N_{f}}\right\} & : \mathcal{F}^{(0)} \longrightarrow \mathcal{F}^{\left(N_{f}\right)} \\
\widehat{Q}_{N_{f}-2}=\left\{\widehat{\psi}_{+}^{1} \widehat{\psi}_{+}^{2} \ldots \widehat{\psi}_{+}^{i-1} \widehat{\psi}_{-}^{i} \widehat{\psi}_{+}^{i+1} \ldots \widehat{\psi}_{+}^{N_{f}}\right\} & : \mathcal{F}^{(1)} \longrightarrow \mathcal{F}^{\left(N_{f}-1\right)}
\end{array}
$$

Clearly, these operators do not commute with the Hamiltonian, for example,

$$
\left[\widehat{N}, \widehat{Q}_{N_{f}}\right]=N_{f} \widehat{Q}_{N_{f}}, \quad\left[\widehat{H}, \widehat{Q}_{N_{f}}\right]=N_{f} \mathcal{W}^{\prime \prime} \widehat{Q}_{N_{f}}
$$

On the other hand, the operators $P \widehat{Q}_{N_{f}}, P \widehat{Q}_{N_{f}-2}, \ldots$ are nilpotent and they commute with the Hamiltonian,

$$
\left(P \widehat{Q}_{N_{f}}\right)^{2}=\left(P \widehat{Q}_{N_{f}-2}\right)^{2}=\ldots=0 \quad \text { and } \quad\left[\widehat{H}, P \widehat{Q}_{N_{f}}\right]|\Psi\rangle=0 \quad \forall|\Psi\rangle \in \mathcal{H}
$$

Note that, for odd (even) $N_{f}, P \widehat{Q}_{N_{f}}, P \widehat{Q}_{N_{f}-2}, \ldots$ pair up bosonic states with fermionic (bosonic) states. See Figure 6 for a demonstration in the cases $N_{f}=2$ and $N_{f}=3$. 
Use of analytic continuation in conjunction with isospectrality The isospectrality will be useful in path integration as well. The partition functions of the isospectral pairs are obviously identical. In the path integral picture, there is a trivial change of variable which takes one path integral to the other, by $\mathfrak{x}(t) \rightarrow-\mathfrak{x}(t)$ for the double-well, or a shift $\frac{\mathfrak{x} \sqrt{g}}{2 a} \sim \frac{\mathfrak{x} \sqrt{g}}{2 a}+\pi$ for the periodic potential.

Alternatively, one can also view these paired systems as follows: Consider the analytic continuation of the potential in sector $k,(2.16)$, into $V(x)=\frac{1}{2}\left(\mathcal{W}^{\prime}\right)^{2}+(k-$ $\left.\frac{N_{f}}{2}\right) e^{i \theta} g W^{\prime \prime}$, where we inserted $e^{i \theta}$ into the fermion induced term. Clearly, interpolating in $\theta$ from $\theta=0$ to $\theta=\pi$ interchanges the two potentials, Hamiltonians, and Hilbert spaces:

$$
\left(\widehat{H}_{k}, \mathcal{H}_{k}\right)(\theta=\pi)=\left(\widehat{H}_{N_{f}-k}, \mathcal{H}_{N_{f}, k}\right)(\theta=0)
$$

When we discuss exact saddles, we will often find one simple bounce solution in one of the mirror pairs (it does not matter which) which is related to the excited state, and by analytic continuation in $\theta$ all the way to $\pi$, we will land on the more exotic complex bion saddles on the mirror which determines the properties of the ground state! This is discussed in depth in section 5 and 6.

\section{Original formulation and local-global relations}

Outline: In this section we provide an analysis of the problem using conventional methods: the BPS-equations, instantons, local harmonic analysis, and index theorems. The non-perturbative contribution to the ground state energy in multi-flavor theories $N_{f} \geq 1$ is always a two-instanton effect (unlike the symmetric DW, where it is a one-instanton effect). We also build up a Hilbert space interpretation for the ground states in terms of local harmonic states, which is again different from the symmetric DW.

\subsection{Auxiliary Potential}

We first consider a general auxiliary potential $\mathcal{W}(\mathfrak{x})$. For the $N_{f}=1$, supersymmetric, theory it coincides with the superpotential. Our goal is to give an intuitive explanation of certain local vs. global relations, which have close relations with real Morse theory, but are fairly easy to explain in physical terms. In the case of a $\mathcal{W}(\mathfrak{x})$ for which the bosonic potential $V_{\text {bos }}=\frac{1}{2}\left(\mathcal{W}^{\prime}\right)^{2}$ has local harmonic minima, there are deep connections between certain local and global properties of the system.

- Two consecutive ground states in harmonic approximation always alternate, $|0\rangle \otimes$ $|\Omega\rangle$ vs. $|0\rangle \otimes|\widetilde{\Omega}\rangle$, where $|\Omega\rangle$ is unoccupied and $|\widetilde{\Omega}\rangle$ is the fully occupied state. 
- The index for Dirac operator for two consecutive instantons ${ }^{10}$ always alternate in sign.

- These two statements are related to the finiteness of the fermionic Fock space, the absence of a fermion number anomaly, and the absence of a Dirac sea in quantum mechanics.

It may appear surprising that the perturbative vacuum structure in the harmonic approximation "knows" about the index theorem and its implications. This, too, is related to the information encoded in $\mathcal{W}^{\prime}(\mathfrak{x})$ and to real Morse theory. We will not dwell on this subject here. Let us first start with local harmonic analysis.

\subsection{Local harmonic analysis, states, and auxiliary Morse function}

In order to understand the connection between the local harmonic analysis of the Hilbert space at each minimum and the index of the Dirac operator it is useful to realize that the auxiliary potential $\mathcal{W}(\mathfrak{x})$ is a Morse function, a real-valued smooth function of Euclidean time. Let us further choose the potential to be a square-free polynomial:

$$
V_{\text {bos }}(\mathfrak{x})=\frac{1}{2}\left(\mathcal{W}^{\prime}\right)^{2}, \quad \mathcal{W}^{\prime}(\mathfrak{x})=\left(\mathfrak{x}-\mathfrak{x}_{1}\right) \ldots\left(\mathfrak{x}-\mathfrak{x}_{n}\right), \quad \mathfrak{x}_{1}<\mathfrak{x}_{2}<\ldots<\mathfrak{x}_{n}
$$

A typical potential $V_{\text {bos }}(\mathfrak{x})$ is shown in Figure 7 . At first glance, all the minima $\mathfrak{x}_{i}$ of the potential $V(\mathfrak{x})$ may be viewed on the same footing, and in fact, in a purely bosonic theory, this is the case. But even at the level of the harmonic approximation, in theories with fermions, there are differences between consecutive minima. This difference is best described if one plots the auxiliary potential $\mathcal{W}(\mathfrak{x})$, which we view as a real Morse function. The extrema (minima and maxima) of $\mathcal{W}(\mathfrak{x})$ are the zeros of the potential $V_{\text {bos }}(\mathfrak{x})$, and there are differences in the description of states depending on $\mathfrak{x}_{i}$ being a local minimum or maximum of the auxiliary function $\mathcal{W}(\mathfrak{x})$. A typical auxiliary potential and its bosonic potential are shown in Figure 7.

Evaluating the Hamiltonian at the $i^{\text {th }}$ harmonic minimum to quadratic order, we

\footnotetext{
${ }^{10}$ We take the definition of instantons as right tunneling events, and anti-instanton as left tunneling. See Section 3.3 for details.
} 

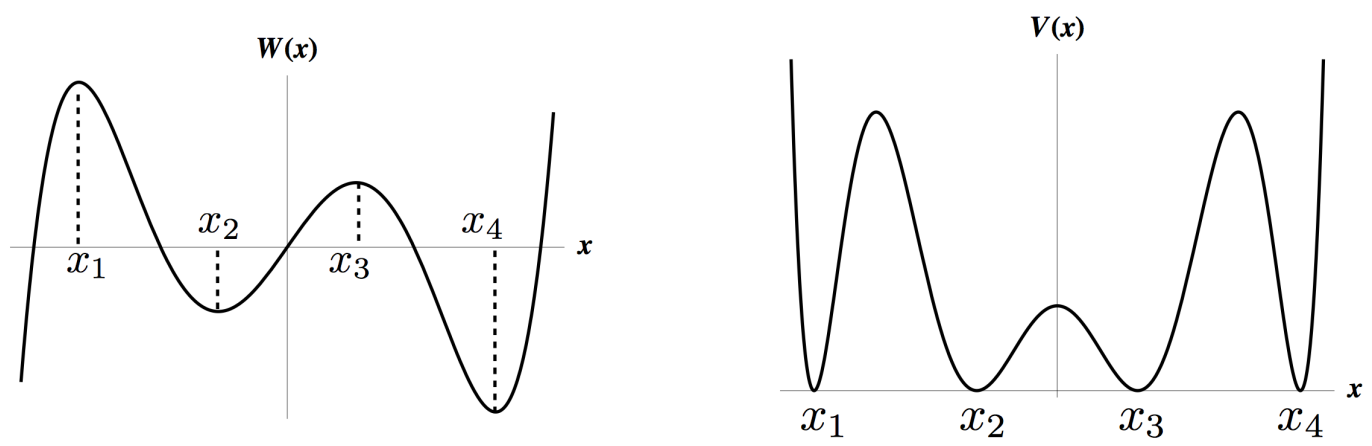

Figure 7. A typical auxiliary potential $\mathcal{W}(\mathfrak{x})$ and associated real potential $V_{\text {bos }}(\mathfrak{x})=$ $\frac{1}{2}\left(\mathcal{W}^{\prime}(\mathfrak{x})\right)^{2}$. The auxiliary potential (which may also be viewed as a Morse function) is useful in understanding local harmonic analysis of Hilbert spaces at each well, and in the study of the index for the Dirac operator in the presence of an instanton. These two (naively) different type data must be related because of the absence of Dirac sea and absence of fermion number anomaly in quantum mechanics.

find that

$$
\begin{aligned}
\widehat{H}_{i} & =\frac{1}{2} \mathfrak{p}^{2}+\frac{1}{2}\left(\mathcal{W}^{\prime \prime}\left(\mathfrak{x}_{i}\right)\right)^{2}(\delta \mathfrak{x})^{2}+\left(\operatorname{sign} \mathcal{W}^{\prime \prime}\left(\mathfrak{x}_{i}\right)\right)\left|\mathcal{W}^{\prime \prime}\left(\mathfrak{x}_{i}\right)\right| \sum_{i=1}^{N_{f}}\left(\widehat{\psi}_{+}^{i} \widehat{\psi}_{-}^{i}-\frac{1}{2}\right) \\
& =\omega_{i}\left[a_{b}^{\dagger} a_{b}+\frac{1}{2}+(-1)^{\mu_{i}}\left(\widehat{N}_{f}-\frac{1}{2} N_{f}\right)\right], \quad \omega_{i}=\left|\mathcal{W}^{\prime \prime}\left(x_{i}\right)\right| \\
& =\omega_{i} \begin{cases}{\left[\widehat{N}_{b}-\widehat{N}_{f}+\frac{N_{f}+1}{2}\right]} & \text { if } \mu_{i}=1, \\
{\left[\widehat{N}_{b}+\widehat{N}_{f}+\frac{1-N_{f}}{2}\right]} & \text { if } \mu_{i}=0,\end{cases}
\end{aligned}
$$

where $\omega_{i}$ is the natural frequency of the harmonic oscillator at $i^{\text {th }}$ minimum and $\mu_{i}$ is the Morse index (number of downward flow directions), which is one if $\mathfrak{x}_{i}$ is a maximum, and zero if it is a minimum. The crucial point is the appearance of $\left.(-1)^{\mu_{i}}=\operatorname{sign} \mathcal{W}^{\prime \prime}\left(\mathfrak{x}_{i}\right)\right)$ in the local quadratic Hamiltonian. For a real polynomial $\mathcal{W}(\mathfrak{x})$, two consecutive extrema are of opposite nature, thus, $\operatorname{sign}\left(\mathcal{W}^{\prime \prime}\left(\mathfrak{x}_{i}\right)\right)$ alternates in its sign. This implies that the ground states in two consecutive vacua are also alternating:

local harmonic ground states $= \begin{cases}|0\rangle \otimes|\widetilde{\Omega}\rangle=|0\rangle \otimes|\uparrow \ldots \uparrow\rangle & \text { for } \mu_{i}=1, \\ |0\rangle \otimes|\Omega\rangle=|0\rangle \otimes|\downarrow \ldots \downarrow\rangle & \text { for } \mu_{i}=0 .\end{cases}$ 


\subsection{Instantons and index theorem for Dirac operator}

Instantons are tunneling events that involve transitions between two critical points, say, $x_{i}$ to $x_{i+1}$, of $W(x)$. We use physics conventions for instantons, i.e., we refer to right tunneling events along which $x(t)$ increases in the $-\infty<t<\infty$ as "instantons". Consequently, our instanton obeys:

$$
\begin{array}{lllll}
\dot{x}=-W^{\prime}(x) & \text { if } & W^{\prime}(x)<0 & \text { for } & x_{i}<x<x_{i+1}, \\
\dot{x}=+W^{\prime}(x) & \text { if } & W^{\prime}(x)>0 & \text { for } & x_{i}<x<x_{i+1},
\end{array}
$$

so that the right hand side is always positive and hence, $x(t)$ increases with $t$. Our anti-instantons are left tunneling events, with opposite signs. ${ }^{11}$ The action of these instanton events is given by

$$
g S_{I, i}=\left|\int_{x_{i}}^{x_{i+1}} d W\right|=\left|W\left(x_{i+1}\right)-W\left(x_{i}\right)\right|,
$$

which is just the difference of the magnitude of the auxiliary Morse function $W(x)$ at two consecutive points.

In this work we consider a cubic auxiliary potential $W(x)$ for which the bosonic potential $V_{\text {bos }}(x)$ is the standard double-well potential, as well as a cosine potential $W(x)$ which leads to the Sine-Gordon bosonic potential. Using non-perturbative normalization, see Footnote. 5,

$$
\begin{aligned}
& W(x)=\frac{x^{3}}{3}-a^{2} x, \\
& W(x)=4 a^{3} \cos \left(\frac{x}{2 a}\right) .
\end{aligned}
$$

In non-perturbative normalization, the first order BPS equation and its solution are independent of the coupling $g$. The smooth instanton solutions and their actions are given by

$$
\begin{aligned}
& \mathrm{DW}: \quad x_{\mathcal{I}}(t)=a \tanh \frac{m_{b}}{2}\left(t-t_{c}\right), \quad m_{b}=2 a, \quad S_{I}=\frac{4 a^{3}}{3 g}, \\
& \mathrm{SG}: \quad x_{\mathcal{I}}(t)=4 a \arctan \left(\exp \left[m_{b}\left(t-t_{c}\right)\right]\right), \quad m_{b}=a, \quad S_{I}=\frac{8 a^{3}}{g} .
\end{aligned}
$$

\footnotetext{
11 According to (real) Morse theory, if the flow from the critical point $x_{i}$ to $x_{i+1}$ is down-ward flow, the flow from the critical point $x_{i+1}$ to $x_{i+2}$ must be an upward flow. This is the reason for the sign in our instanton equation. Otherwise, if we take a uniform sign convention for instantons, two consecutive right tunneling events would be called instanton and anti-instanton.
} 
$t_{c} \in \mathbb{R}$ is the position modulus, a bosonic zero mode of the instanton solution. The parameter $m_{b}$ is the mass parameter, which is the natural frequency of small oscillations around the harmonic minima.

The instanton amplitude is given by

$$
\mathcal{I}=J_{t_{c}} e^{-S_{\mathcal{I}}}\left[\frac{\operatorname{det}^{\prime} M}{\operatorname{det} M_{0}}\right]^{-\frac{1}{2}}= \begin{cases}\sqrt{\frac{6 S}{\pi}} e^{-S} & (\mathrm{DW}) \\ \sqrt{\frac{2 S}{\pi}} e^{-S} & (\mathrm{SG})\end{cases}
$$

where $J_{t_{c}}=\sqrt{\frac{S}{2 \pi}}$ is the Jacobian associated with the bosonic zero mode, $\mathbf{M}=-\frac{d^{2}}{d t^{2}}+$ $\left.V^{\prime \prime}(x)\right|_{x=x_{\mathcal{I}}(t)}$ is the quadratic fluctuation operator in the background of the instanton and prime in $\operatorname{det}^{\prime} \mathbf{M}$ denotes removal of the zero mode, and $\operatorname{det} M_{0}$ is for normalization. The determinant can be evaluated in multiple different ways [6, 65], for example, via the Gelfand-Yaglom method [66], and gives

$$
\frac{\operatorname{det}^{\prime} M}{\operatorname{det} M_{0}}= \begin{cases}\frac{1}{12} & (\mathrm{DW}), \\ \frac{1}{4} & (\mathrm{SG}),\end{cases}
$$

resulting in the instanton amplitudes given in (3.9).

Fermionic zero modes and index theorem: In the theory with fermions, an instanton has a certain number of fermion zero modes, as a result of an index theorem for the one dimensional Dirac operator. Defining

$$
D=+d_{t}+W^{\prime \prime}\left(x_{I}\right), \quad D^{\dagger}=-d_{t}+W^{\prime \prime}\left(x_{I}\right),
$$

the index is equal to the spectral asymmetry of the Dirac operator. For each flavor of fermions,

$$
\begin{aligned}
n_{\psi}-n_{\bar{\psi}} & =\operatorname{Index}=\operatorname{dim} \operatorname{ker}(D)-\operatorname{dim} \operatorname{ker}\left(D^{\dagger}\right) \\
& =\frac{1}{2}\left[\operatorname{sign}\left\{W^{\prime \prime}\left(x_{I}(t=\infty)\right)\right\}-\operatorname{sign} W^{\prime \prime}\left(x_{I}(t=-\infty)\right)\right] \\
& =\frac{1}{2}\left[(-1)^{\mu_{i}}-(-1)^{\mu_{i+1}}\right] \\
& = \begin{cases}1 & \text { for } \mu_{i}=1, \\
-1 & \text { for } \mu_{i}=0 .\end{cases}
\end{aligned}
$$

Thus, the "instanton amplitudes" differ for an instanton interpolating from $x_{i}$ to $x_{i+1}$ relative to the consecutive instanton interpolating from $x_{i+1}$ to $x_{i+2}$. In particular, one has

$$
\mathcal{I}_{i, i+1} \sim \sqrt{\frac{S_{I}}{2 \pi}} e^{-S_{I} "} \psi_{1} \psi_{2} \ldots \psi_{N_{f}} "
$$




$$
\mathcal{I}_{i+1, i+2} \sim \sqrt{\frac{S_{I}}{2 \pi}} e^{-S_{I}} " \bar{\psi}_{1} \bar{\psi}_{2} \ldots \bar{\psi}_{N_{f}} "
$$

The anti-instanton amplitudes are given by the conjugates of these. We put the annihilation and creation operators $\psi_{i}, \bar{\psi}_{i}$ in quotes is to remind the reader that this is a symbolic notation, inspired by the index theorem. But the meaning is different from QFTs, where instantons induce an anomaly. In quantum mechanics, of course, there is no anomaly, and the instanton amplitudes do not violate fermion number symmetry (or spin), see Section 3.4 .

\subsection{Difference between QFT and QM: Hilbert's hotel or not}

There is a significant difference between the physical implications of the index theorem for the Dirac operator in 1d quantum mechanics compared to QCD or other QFTs. In QCD, in the background of an instanton, there is chiral charge non-conservation, i.e. the $U(1)_{A}$ is anomalous. A left handed particle can annihilate and create a right handed one, violating $N_{R}-N_{L}$ by two units. In the Euclidean formulation, the density of these events is finite, thus, in an infinite volume, this process may happen infinitely many times. This does not lead to any pathology in QFT because there is an (infinite) Dirac sea reservoir for both chiralities, i.e, the Dirac sea is a "Hilbert hotel".

In quantum mechanics, there is no anomaly because of the finiteness of the number of degrees of freedom and ultimately, due to the absence of the Dirac sea. In particular, there is no fermion number anomaly. Despite the fact that the instanton amplitude looks like it violates fermion number by $N_{f}$, this does not imply an anomaly.

Consider QCD with one-flavor Dirac fermion. The instanton reduces the $U(1)_{A}$ symmetry down to $\mathbb{Z}_{2}$ because of the anomaly. In contrast, consider $N_{f}=2 \mathrm{QM}$. The instanton amplitude is formally the same, and one may think that this also reduces $U(1)_{F}$ down to $\mathbb{Z}_{2}$. However, this is incorrect, because of the absence of a fermion number anomaly:

$$
\begin{array}{lll}
\mathcal{I}_{4 d} \sim e^{-S_{I}} \psi_{L} \psi_{R} & \Longrightarrow U(1)_{A} \longrightarrow \mathbb{Z}_{2} & \text { for QCD, } \\
\mathcal{I}_{1 d} \sim e^{-S_{I}} \psi_{1} \psi_{2} & \Rightarrow U(1)_{F} \longrightarrow \mathbb{Z}_{2} & \text { for QM. }
\end{array}
$$

However while in QCD the instanton vertex $\psi_{L} \psi_{R}$ is a genuine operator accompanying an instanton in the low energy effective theory, in QM writing $\psi_{1} \psi_{2}$ is symbolic and it implies the existence of two zero-modes. The meaning of $\psi_{1} \psi_{2}$ is that the instanton saturates a matrix element connecting states whose fermion number differs by two units.

Further, let $\left|G_{i}\right\rangle$ denote a ground state of either theory. Then the difference between QFT and QM is that in QCD, for the present example of 1-flavor QCD, the ground 
state is unique, and $U(1)_{A}$ breaking operators have a non-zero expectation value in the ground state. In QM, on the other hand, there is more than one ground state. The operator charged under $U(1)_{F}$ has vanishing matrix elements in each of the ground states. Instead, the operator $\widehat{\psi}_{1} \widehat{\psi}_{2}$ has non-zero matrix elements between states whose fermion number differs by two units.

$$
\begin{array}{ll}
\text { for QCD : } & \left\langle\mathrm{G}_{i}\left|\widehat{\psi}_{L} \widehat{\psi}_{R}\right| \mathrm{G}_{i}\right\rangle \neq 0, \\
\text { for QM : } \quad & \left\{\begin{array}{l}
\left\langle\mathrm{G}_{i}\left|\widehat{\psi}_{1} \widehat{\psi}_{2}\right| \mathrm{G}_{i}\right\rangle=0, \\
\left\langle\mathrm{G}_{j}\left|\widehat{\psi}_{1} \widehat{\psi}_{2}\right| \mathrm{G}_{i}\right\rangle \neq 0 . \quad \exists(i, j), \quad(i \neq j) .
\end{array}\right.
\end{array}
$$

Indeed, in the semi-classical approximation, two consecutive harmonic vacua are, respectively, full and empty. The alternating vacuum structure and alternating index theorem conspire to give an alternating harmonic vacuum chain among the perturbative vacuum states:

$$
\underbrace{\longrightarrow}_{\mathcal{I} \sim \bar{\psi}_{1} \ldots \bar{\psi}_{N_{f}}}|0\rangle_{i} \otimes|\widetilde{\Omega}\rangle_{i} \underbrace{\longrightarrow}_{\mathcal{I}_{i} \sim \psi_{1} \ldots \psi_{N_{f}}}|0\rangle_{i+1} \otimes|\Omega\rangle_{i+1} \underbrace{\longrightarrow}_{\mathcal{I}_{i+1} \sim \bar{\psi}_{1} \ldots \bar{\psi}_{N_{f}}}|0\rangle_{i+2} \otimes|\widetilde{\Omega}\rangle_{i+2} \underbrace{\longrightarrow}_{\mathcal{I} \sim \psi_{1} \ldots \psi_{N_{f}}}
$$

Starting with a fully occupied harmonic state an instanton event in QM is associated with a transition matrix element with the insertion of operator $\widehat{\psi}_{1} \ldots \widehat{\psi}_{N_{f}}$ for which the fermion number changes by $N_{f}$. Thus, the subsequent state is empty. The next instanton event is associated with a fermion number increasing matrix element, and refills all $N_{f}$ unoccupied states, and so and so forth. One cannot have two consecutive instanton events where all are reducing the fermion number. This clashes with finiteness of the Fermion Fock space. Unlike the chiral charge in QCD which does not commute with the QCD Hamiltonian, the fermion number operator (i.e. z-component of Spin) commutes with the Hamiltonian (2.5). As a result, there are a multitude of interesting and somehow unconventional instanton effects in this class of quantum mechanical theories, discussed in the next section.

To summarize, we show that the following three concepts are intertwined:

- Finiteness of Fock space, or absence of Dirac sea.

- Alternating index for instantons, absence of fermion number anomaly.

- Alternating fermion numbers in perturbative vacua in harmonic approximation. 


\subsection{Mixing of harmonic states in QM with fermions: New instanton effects}

For simplicity, we discuss a particle with internal spin $\left(\frac{1}{2}\right)^{N_{f}}$ in a DW potential. This has almost all interesting features that also takes place for more general potentials.

$N_{f}=\mathbf{0}$ (reminder): The theory is bosonic and the states on either well are harmonic oscillator eigenstates, $|L, n\rangle$ and $|R, n\rangle$. These states are degenerate to all orders in perturbation theory. Non-perturbatively, the degeneracy between $|L, 0\rangle$ and $|R, 0\rangle$ is lifted due to tunneling/instanton events. The ground state is the parity even combination and the first excited state is the parity odd combination of the two lowest lying modes. These are

$$
\left|\Psi_{\epsilon}\right\rangle=\frac{1}{\sqrt{2}}(|L, 0\rangle+\epsilon|R, 0\rangle), \quad \mathbf{P}\left|\Psi_{\epsilon}\right\rangle=\epsilon\left|\Psi_{\epsilon}\right\rangle, \quad \epsilon= \pm
$$

where $\mathbf{P}$ is the parity operator. The tunneling amplitude is equal to the instanton amplitude given in (3.9):

$$
\left\langle-a\left|e^{-T H}\right| a\right\rangle=\mathcal{N} \mathcal{I}=\mathcal{N} \sqrt{\frac{6 S_{I}}{\pi}} e^{-S_{I}}
$$

where $\mathcal{N}=\frac{1}{2}\langle R, 0 \mid a\rangle^{2}(\omega T) e^{-\omega T}$. Consequently, the non-perturbative splitting or lifting

of the perturbative two-fold degeneracy is an instanton effect $\left(E_{-}-E_{+}\right) / \omega=\sqrt{\frac{6 S_{I}}{\pi}} e^{-S_{I}}$ at leading non-perturbative order. The reason for restating this well-known result is that instantons do not lead to the level splitting effects in theories with $N_{f} \geq 1$, as described below.

$N_{f}=1$ (supersymmetric) theory: In the harmonic approximation the states in both the left or the right well are described by the Hilbert space of supersymmetric harmonic oscillator. The lowest lying states in $\mathcal{H}_{L}$ and $\mathcal{H}_{R}$ as well as the corresponding eigen-energies are:

$$
\begin{array}{lll}
E_{3}=3 & \overbrace{|L, 2\rangle|0\rangle}^{\mathcal{B}} \longleftrightarrow \overbrace{|L, 3\rangle|1\rangle}^{\mathcal{F}} & \overbrace{|R, 3\rangle|0\rangle}^{\mathcal{B}} \longleftrightarrow \overbrace{|R, 2\rangle|1\rangle}^{\mathcal{F}} \\
E_{2}=2 & |L, 1\rangle|0\rangle \longleftrightarrow|L, 2\rangle|1\rangle & |R, 2\rangle|0\rangle \longleftrightarrow|R, 1\rangle|1\rangle \\
E_{1}=1 & |L, 0\rangle|0\rangle \longleftrightarrow|L, 1\rangle|1\rangle & |R, 1\rangle|0\rangle \longleftrightarrow|R, 0\rangle|1\rangle \\
E_{0}=0 & \underbrace{|L, 0\rangle|1\rangle}_{\mathcal{H}_{L}} & \underbrace{|R, 0\rangle|0\rangle}_{\mathcal{H}_{R}}
\end{array}
$$

In the supersymmetric theory, the two perturbative ground states $|L, 0\rangle|1\rangle$ and $|R, 0\rangle|0\rangle$ cannot mix because of the conservation of fermion number or spin. As emphasized in Sec.3.4, there is no fermion number anomaly, i.e., fermion number operator commutes with the Hamiltonian:

$$
\left[H, \widehat{N}_{f}\right]=\left[H, \sigma_{3}\right]=0
$$


Thus, the matrix element of $e^{-T H}$ connecting left harmonic ground state to right one vanishes:

$$
\left\langle-a\left|\left\langle 1\left|e^{-T H}\right| a\right\rangle\right| 0\right\rangle=0
$$

in contrast to the bosonic case (3.18), which implies that there is no splitting of the two lowest lying states. Instead, the energy of both states gets simultaneously lifted, and because of supersymmetry the shift is exactly the same for both states. To leading order in $\hbar$, the lifting is due to mixing between the harmonic ground state of $\mathcal{H}_{L}$, and the first excited state of $\mathcal{H}_{R}$. For the other ground state the role of $L$ and $R$ is exchanged.

Therefore, in terms of the harmonic states of the left and right well, the two nonperturbative ground state wave functions are given by

$$
\begin{aligned}
& \left|\Psi_{0}\right\rangle|1\rangle=a_{1} \underbrace{|R, 0\rangle|0\rangle}_{\text {Ground state in } \mathcal{H}_{\mathrm{L}} \underbrace{|L, 0\rangle|1\rangle}_{\text {First-excited state in } \mathcal{H}_{\mathrm{R}}}+a_{2} \underbrace{|R, 0\rangle|1\rangle}_{\text {Ground state in } \mathcal{H}_{\mathrm{R}}}+\ldots}+a_{2} \underbrace{|L, 0\rangle|0\rangle}_{\text {First-excited state in } \mathcal{H}_{\mathrm{L}}}+\ldots
\end{aligned}
$$

Unlike the bosonic system in which the ground state must be unique, in the theory with fermions, the ground state is two-fold degenerate. The ground state energy can be found by calculating the expectation value of the Hamiltonian in either one of the two true ground states. We have

$$
\begin{aligned}
E_{0} & =\left\langle\Psi_{0}|\langle 0|\widehat{H}| 0\rangle| \Psi_{0}\right\rangle=\frac{1}{2}\left\langle\Psi_{0}|\langle 0|\{Q, \bar{Q}\}| 0\rangle| \Psi_{0}\right\rangle=\frac{1}{2}\left\langle\Psi_{0}|\langle 0|Q \bar{Q}| 0\rangle| \Psi_{0}\right\rangle= \\
& =\frac{1}{2}\left\langle\Psi _ { 0 } \left|\left\langle0 \left|Q \left(\sum_{n, \sigma=0,1}|\sigma\rangle\left|\Psi_{n}\right\rangle\left\langle\Psi_{n}|\langle\sigma|) \bar{Q} \mid 0\right\rangle\left|\Psi_{0}\right\rangle=\frac{1}{2} \sum_{n}\left|\left\langle\Psi_{n}|\langle 1|\bar{Q}| 0\rangle| \Psi_{0}\right\rangle\right|^{2}\right.\right.\right.\right.\right. \\
& \approx \frac{1}{2}\left|\left\langle\Psi_{0}|\langle 1|\bar{Q}| 0\rangle| \Psi_{0}\right\rangle\right|^{2},
\end{aligned}
$$

where $\bar{Q}=\left(p+i W^{\prime}\right) \widehat{\psi}_{+}$and $Q=\left(p-i W^{\prime}\right) \widehat{\psi}_{-}$are the supercharges, and in the final line we inserted a complete set of states. Note that since $\bar{Q}$ commutes with Hamiltonian, but anti-commutes commute with $(-1)^{F}$, the sum over $\sigma$ reduces to $\sigma=1$. For the final estimate, we kept only the dominant $n=0$ state in the sum.

The ground-state energy is therefore the square of a matrix element, $E_{0}=\frac{1}{2}|\epsilon|^{2}$, where $\epsilon=\left\langle\Psi_{0}|\langle 1|\bar{Q}| 0\rangle| \Psi_{0}\right\rangle$. This is the quantity saturated by an instanton. Instanton processes are associated with the matrix element $\left\langle\Psi_{0}|\langle 1|\bar{Q}| 0\rangle| \Psi_{0}\right\rangle$ between the two ground states, which have different fermion numbers (or spin). In the functional integral formulation, this result can be written as:

$$
\left\langle\Psi_{0}|\langle 1|\bar{Q}| 0\rangle| \Psi_{0}\right\rangle \sim\langle-a|\langle 1|\bar{Q}|+a\rangle| 0\rangle \sim \int_{x(-\infty)=-a}^{x(\infty)=+a} D x D \psi D \bar{\psi} e^{-S}\left(p+i W^{\prime}\right) \psi .(
$$



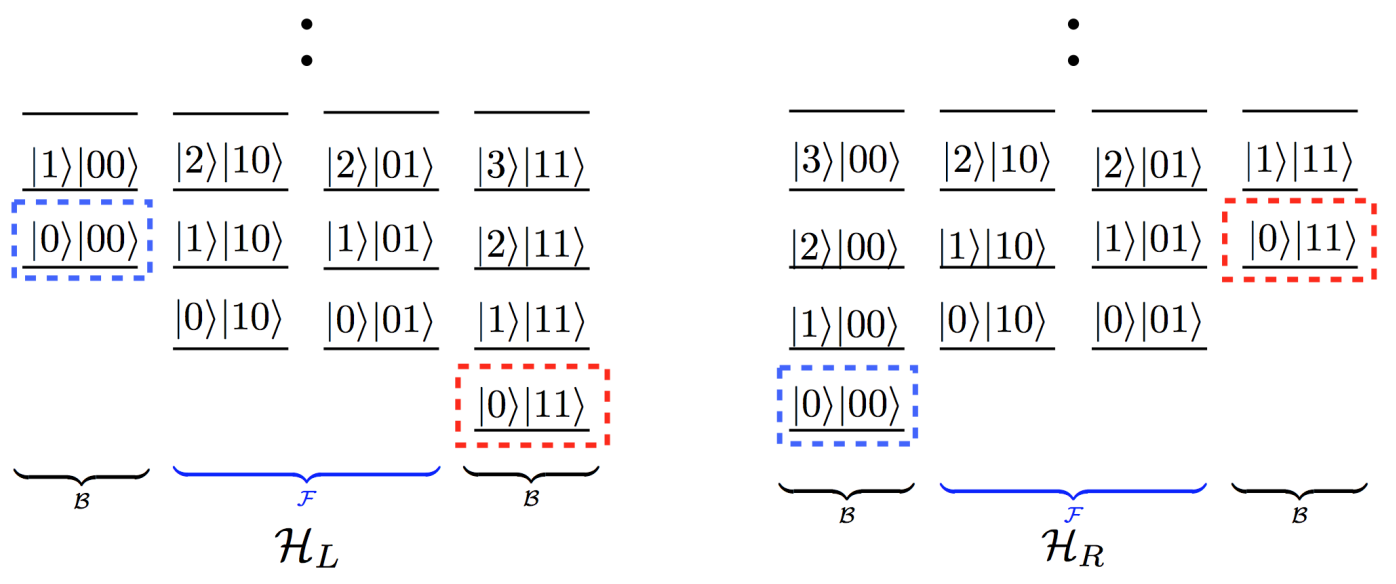

Figure 8. Hilbert-space on the left and right well in the $N_{f}=2$ (multi-flavor) theory in harmonic approximation. Perturbative ground state on the left well is fully occupied, while the one on the right is empty. These two cannot mix (form linear combinations) due to conservation of fermion number or spin. Non-perturbatively, the theory has two ground states. One is (approximately) a linear superposition of ground state on the left and second excited state on the right (with the same fermion occupation number, the ones in red boxes), and the other is the linear superposition of the states in blue boxes.

The evaluation of the integral gives $\epsilon \sim e^{-S_{I}}$. See [67] for details. The shift in the ground state energy is

$$
E_{0} \sim e^{-2 S_{I}}
$$

This is a second order effect in semi-classical expansion, and it can be interpreted in terms of a dilute gas of correlated instanton-anti-instanton $[\mathcal{I} \overline{\mathcal{I}}]$ events. However, this formulation does not tell us if the $[\mathcal{I} \overline{\mathcal{I}}]$ configuration is an exact solution or not. In fact, there is reason to think otherwise. Since the BPS equations are non-linear, the superposition of the two configuration is not a solution to that BPS equation, i.e, the configuration is non-BPS.

In the graded formulation, we will prove that an exact non-BPS complex saddle is responsible for the ground state energy (3.25), and that $[\mathcal{I} \overline{\mathcal{I}}]$ (evaluated on appropriate complex quasi-zero mode thimble, not over the naive "separation" cycle) is an approximation to the exact solution. We will also show that the exact saddles are solutions to holomorphic and quantum modified second order Euclidean equations of motions.

$N_{f} \geq 2$ (non-supersymmetric, multi-flavor) theory: In harmonic approximation the states in the left and right well are described by a natural generalization of the Hilbert space of the supersymmetric harmonic oscillator. Our construction follows [68]. For the free theory, which is relevant in the case of the harmonic approximation, 
one can define $N_{f}$ nilpotent conserved fermionic charges:

$$
Q_{i}=a_{b}^{\dagger} \widehat{\psi}_{-}^{i}, \quad Q_{i}^{2}=0
$$

Let $|\psi\rangle=|n\rangle|\Omega\rangle$ be an eigenstate of the Hamiltonian $\widehat{H}_{R}$ with energy $E_{n}$ annihilated by $Q_{i}, \forall i=1, \ldots, N_{f}$. It is possible to generate all states in the right harmonic Hilbert space $\mathcal{H}_{R}$ by acting with the $Q_{i}^{\dagger}$ 's on $|\psi\rangle$. Consider the set:

$$
S=\left\{|\psi\rangle, Q_{i}^{\dagger}|\psi\rangle, Q_{i}^{\dagger} Q_{j}^{\dagger}|\psi\rangle, \ldots, Q_{i}^{\dagger} \ldots Q_{i_{N_{f}}}^{\dagger}|\psi\rangle\right\}
$$

If $n \geq N_{f}$, this procedure, which goes parallel to our construction of the fermionic Fock space in Section 2.2, creates $2^{N_{f}}$ degenerate states at each level: If $n<N_{f}$, some combination of the operators $Q_{i_{1}}^{\dagger} \ldots Q_{i_{k}}^{\dagger}$ annihilates $|\psi\rangle$. For example, in the case $N_{f}=2$, the lowest lying states in $\mathcal{H}_{L}$ and their eigen-energies are given by:

$$
\begin{array}{rrr}
E_{3}=+\frac{5}{2} & |L, 1\rangle|00\rangle \longleftrightarrow|L, 2\rangle|10\rangle \longleftrightarrow|L, 2\rangle|01\rangle \longleftrightarrow|L, 3\rangle|11\rangle \\
E_{2}=+\frac{3}{2} & |L, 0\rangle|00\rangle \longleftrightarrow|L, 1\rangle|10\rangle \longleftrightarrow|L, 1\rangle|01\rangle \longleftrightarrow|L, 2\rangle|11\rangle \\
E_{1}=+\frac{1}{2} & |L, 0\rangle|10\rangle \longleftrightarrow|L, 0\rangle|01\rangle \longleftrightarrow|L, 1\rangle|11\rangle \\
E_{0}=-\frac{1}{2} & & |L, 0\rangle|11\rangle
\end{array}
$$

and analogously, the eigen-states in $\mathcal{H}_{R}$ and their eigen-energies are

$$
\begin{aligned}
& E_{3}=+\frac{5}{2} \quad|R, 3\rangle|00\rangle \longleftrightarrow|R, 2\rangle|10\rangle \longleftrightarrow|R, 2\rangle|01\rangle \longleftrightarrow|R, 1\rangle|11\rangle \\
& E_{2}=+\frac{3}{2} \quad|R, 2\rangle|00\rangle \longleftrightarrow|R, 1\rangle|10\rangle \longleftrightarrow|R, 1\rangle|01\rangle \longleftrightarrow|R, 0\rangle|11\rangle \\
& E_{1}=+\frac{1}{2} \quad|R, 1\rangle|00\rangle \longleftrightarrow|R, 0\rangle|10\rangle \longleftrightarrow|R, 0\rangle|01\rangle \\
& E_{0}=-\frac{1}{2} \quad|R, 0\rangle|00\rangle .
\end{aligned}
$$

As in the $N_{f}=1$ supersymmetric theory, for $N_{f}>1$ as well, the two perturbative ground states $|L, 0\rangle|\widetilde{\Omega}\rangle$ and $|R, 0\rangle|\Omega\rangle$ cannot mix because of the conservation of fermion number (3.20). Therefore, the transition matrix element vanishes:

$$
\left\langle-a\left|\left\langle\widetilde{\Omega}\left|e^{-T H}\right| a\right\rangle\right| \Omega\right\rangle=0, \quad \forall N_{f} \geq 1 .
$$

Similar to the supersymmetric theory, and again unlike the bosonic theory, this implies that there will be no splitting of the two lowest lying states. Instead, the energy of both states gets simultaneously lifted, and they do get lifted exactly by the same amount.

To leading order in $\hbar$, the lifting is due to mixing between the ground state of $\mathcal{H}_{L}$, $|L, 0\rangle|\widetilde{\Omega}\rangle$ and the $N_{f}^{\text {th }}$ excited state of $\mathcal{H}_{R},|R, 0\rangle|\widetilde{\Omega}\rangle$, and vice versa for the other ground state. ${ }^{12}$ Therefore, the two non-perturbative ground state wave functions are

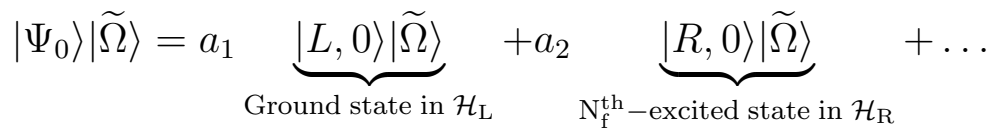

\footnotetext{
${ }^{12}$ The mixing between the lowest state on one side with the $N_{f}^{\text {th }}$ excited state on the other side is also present for the $N_{f}=0$ (bosonic) and $N_{f}=1$ (supersymmetric) examples. For general $N_{f}$, this is a new result.
} 


$$
\left|\Psi_{0}\right\rangle|\Omega\rangle=a_{1} \underbrace{|R, 0\rangle|\Omega\rangle}_{\text {Ground state in } \mathcal{H}_{\mathrm{R}}}+a_{2} \underbrace{|L, 0\rangle|\Omega\rangle}_{\mathrm{N}_{\mathrm{f}}^{\text {th }} \text { - excited state in } \mathcal{H}_{\mathrm{L}}}+\ldots
$$

Unlike the bosonic system in which the ground state must be unique, for the theory with fermions, the ground state is two-fold degenerate. The ground state energy can be found by calculating the expectation value of the Hamiltonian in either one of the two true ground states.

In contrast to the supersymmetric theory, the shift in the ground state energy has perturbative and non-perturbative contributions. We have

$$
E_{0}=\left\langle\Psi_{0}|\langle\Omega|\widehat{H}| \Omega\rangle| \Psi_{0}\right\rangle=E_{0}^{\text {pert }}(g)+E_{0}^{\text {non-pert }}(g)
$$

where the non-perturbative part is a second order effect in the semi-classical expansion,

$$
E_{0}^{\text {non-pert }}(g) \sim\left|\left\langle\Psi_{0}\left|\left\langle\widetilde{\Omega}\left|\bar{Q}_{N_{f}}\right| \Omega\right\rangle\right| \Psi_{0}\right\rangle\right|^{2}
$$

given by a square of a matrix element, $\epsilon=\left\langle\Psi_{0}\left|\left\langle\widetilde{\Omega}\left|\bar{Q}_{N_{f}}\right| \Omega\right\rangle\right| \Psi_{0}\right\rangle$. Because the instanton contributes to a fermion number changing matrix element, and because fermion number commutes with Hamiltonian, the non-perturbative contribution to ground state energy can only start at second order in semi-classical expansion. In terms of a functional integral we can write

$$
\begin{aligned}
\epsilon & =\left\langle\Psi_{0}\left|\left\langle\widetilde{\Omega}\left|\widehat{Q}_{N_{f}}\right| \Omega\right\rangle\right| \Psi_{0}\right\rangle \sim\left\langle-a\left|\left\langle\widetilde{\Omega}\left|\widehat{Q}_{N_{f}}\right| \Omega\right\rangle\right|+a\right\rangle \\
& =\int_{x(-\infty)=-a}^{x(\infty)=+a} D x \prod_{i=1}^{N_{f}} D \psi_{i} D \bar{\psi}_{i} e^{-S} \psi_{1} \psi_{2} \ldots \psi_{N_{f}}
\end{aligned}
$$

The evaluation of this integral gives $\epsilon \sim e^{-S_{I}}$, because the $\psi_{1} \psi_{2} \ldots \psi_{N_{f}}$ inversion gets soaked up to the unpaired zero modes in the measure $D \psi_{i}(t) D \bar{\psi}_{i}(t)$. The nonperturbative shift in the ground state energy is

$$
E_{0}^{\text {non-pert }}(g) \sim e^{-2 S_{I}}
$$

Similar to the supersymmetric case, this can be interpreted in terms of a dilute gas of correlated instanton-anti-instanton $[\mathcal{I} \overline{\mathcal{I}}]$ events. Similar to the supersymmetric theory, in the graded formulation, we prove that an exact non-BPS complex saddle is responsible for the non-perturbative lifting of the ground state energy, and that $[\mathcal{I} \overline{\mathcal{I}}]$ is an approximation to the exact solution. 


\section{Approximate versions of exact solutions from quasi-zero mode thimbles}

Outline: We discuss correlated two-instanton events on the complexified quasi-zero mode Lefschetz thimbles. The treatment of the QZM integration over the Lefschetz thimbles represents a reduced version of the complexification of the full field space. The conventional wisdom is that instanton-anti-instanton events can at best be approximate solutions, due to the non-linearity of the underlying BPS-equations. This intuition turns out to be incorrect, as we later find exact saddles. Remarkably, the Lefschetz thimble treatment of the multi-instantons provides a systematic approximation to the exact result and reproduces the important features of the exact solutions discussed in Section 5 and 6 .

\subsection{Multi-instantons and boson and fermion induced interactions}

In the previous section we saw that for $N_{f} \geq 1$ the non-perturbative contribution to the ground state energy is of the form $e^{-2 S_{I}}$, rather than $e^{-S_{I}}$. In the graded theory, we will in fact find an exact non-BPS bion saddle which is responsible for this ground state energy (3.25). However, in order to understand the connection between the exact saddle and the instantons in the original description, we first sketch the calculation of the contribution to correlated 2-events $[\mathcal{I} \mathcal{I}]$ or $[\mathcal{I} \overline{\mathcal{I}}]$ in the original formulation. Note that in the double-well potential we only have $[\mathcal{I} \overline{\mathcal{I}}]$, while in the periodic potential, we have both.

The instanton amplitude is given in (3.13). As discussed earlier, an instanton has an exact position modulus. If we consider a two instanton configuration then the center position of the two is still an exact zero mode, but the relative separation $\tau$ is a quasizero mode. The reason for this fact is the interaction (in the euclidean sense) between the two instantons. The bosonic interaction can be described as due to the exchange of bosonic fluctuations, derived in detail in [6]. The fermion induced interaction can be deduced either by studying the fermionic determinant in the background of an instanton-(anti)instanton pair, or equivalently, by looking to the connected correlator of the fermion zero mode structure $\left\langle\psi_{1}(0) \ldots \psi_{N_{f}}(0) \bar{\psi}_{1}(\tau) \ldots \bar{\psi}_{N_{f}}(\tau)\right\rangle \sim e^{-N_{f} m_{b} \tau}[27$, 52]. Here, instead of these technical arguments, we provide a more intuitive physical description.

An instanton will interact with an anti-instanton due to the spin interaction with the "magnetic field" $W^{\prime \prime}(x)$. The "magnetic field" at the two classical vacua are $W^{\prime \prime}( \pm a)= \pm 2 a= \pm \omega$, where $\omega$ is the natural frequency. The spin-dependent part of the Hamiltonian is $-S_{z} W^{\prime \prime}(x)$, where $S_{z} \in\left[-N_{f} / 2, N_{f} / 2\right]$ is the spin. Therefore the ground state in the left well will have $S_{z}=N_{f} / 2$ (i.e. all spins up) while the ground state on the right will have $s=-N_{f} / 2$ (i.e. all spins down) (see Fig. 8 ). Now let us consider an instanton-anti-instanton pair interpolating from vacuum at $x=-a$ to the vacuum at $x=+a$ and back, and let the time that this configuration spends at $x=+a$ 
be $\tau$. Since the configuration spends an infinite amount of time in the vacuum $x=-a$, the spin $s$ must be $s=+N_{f} / 2$, and it cannot change along the instanton path, as spin is conserved. Then, the spin induced action cost is the penalty for the Euclidean time spent in the "false vacuum". Then the action cost (compared to the vacuum) will be a product of $2 \omega$ (the change in the magnetic field from left well to the right well), the spin $s=N_{f} / 2$ and the time spent in the right well $\tau$. Therefore $S_{\text {int }}=N_{f} \omega \tau$.

We denote the interaction potential between two instantons by $\mathcal{V}_{+}(z)$, and the one between an instanton and anti-instanton by $\mathcal{V}_{-}(z)$.

$$
\mathcal{V}_{ \pm}(\tau)= \pm \frac{A a^{3}}{g} e^{-m_{b} \tau}+N_{f} m_{b} \tau \quad A= \begin{cases}16 & \text { for DW } \\ 32 & \text { for SG. }\end{cases}
$$

In both cases the fermion zero mode induced interaction is attractive. For $\mathcal{V}_{+}(z)$ the boson induced interaction is repulsive, and for $\mathcal{V}_{-}(z)$ the bosonic part is attractive. In the space of fields (or paths), the quasi-zero mode direction is non-Gaussian, and the integral over the QZM needs to be treated exactly in order to obtain the correlated 2-event amplitudes.

\section{2 $[\mathcal{I} \mathcal{I}]$ thimble integration and approximate form of real bion}

The QZM integration for the $[\mathcal{I I}]$ correlated event is

$$
I_{+}\left(N_{f}, g\right)=\int_{\Gamma_{+}^{\mathrm{qzzm}}} d\left(m_{b} \tau\right) e^{-\mathcal{V}_{+}(\tau)}=\int_{\Gamma_{+}^{\mathrm{qzzm}}} d\left(m_{b} \tau\right) e^{-\left(\frac{A a^{3}}{g} e^{-m_{b} \tau}+N_{f} m_{b} \tau\right)},
$$

where $\Gamma_{+}^{\text {qzm }}$ is the QZM-cycle for the QZM-integration, shown in Fig. 9, lower figures. The critical point of the integration is located at

$$
\frac{d \mathcal{V}_{+}(\tau)}{d \tau}=0, \quad \tau^{*}=m_{b}^{-1} \ln \left(\frac{A a^{3}}{g N_{f}}\right) .
$$

The descent manifold (Lefschetz thimble) associated with this critical point is the real axis, $\Gamma_{+}^{\text {qzm }}=\mathbb{R}$, as can be deduced by solving the stationary phase condition

$$
\operatorname{Im}\left[V(\tau)-V\left(\tau^{*}\right)\right]=0 .
$$

Substituting $u=e^{-m_{b} \tau}$, we map the integral to the standard representation of the Gamma-function:

$$
I_{+}\left(N_{f}, g\right)=\int_{0}^{\infty} d u u^{N_{f}-1} e^{-\frac{A a^{3}}{g} u}=\left(\frac{g}{A a^{3}}\right)^{N_{f}} \Gamma\left(N_{f}\right) .
$$



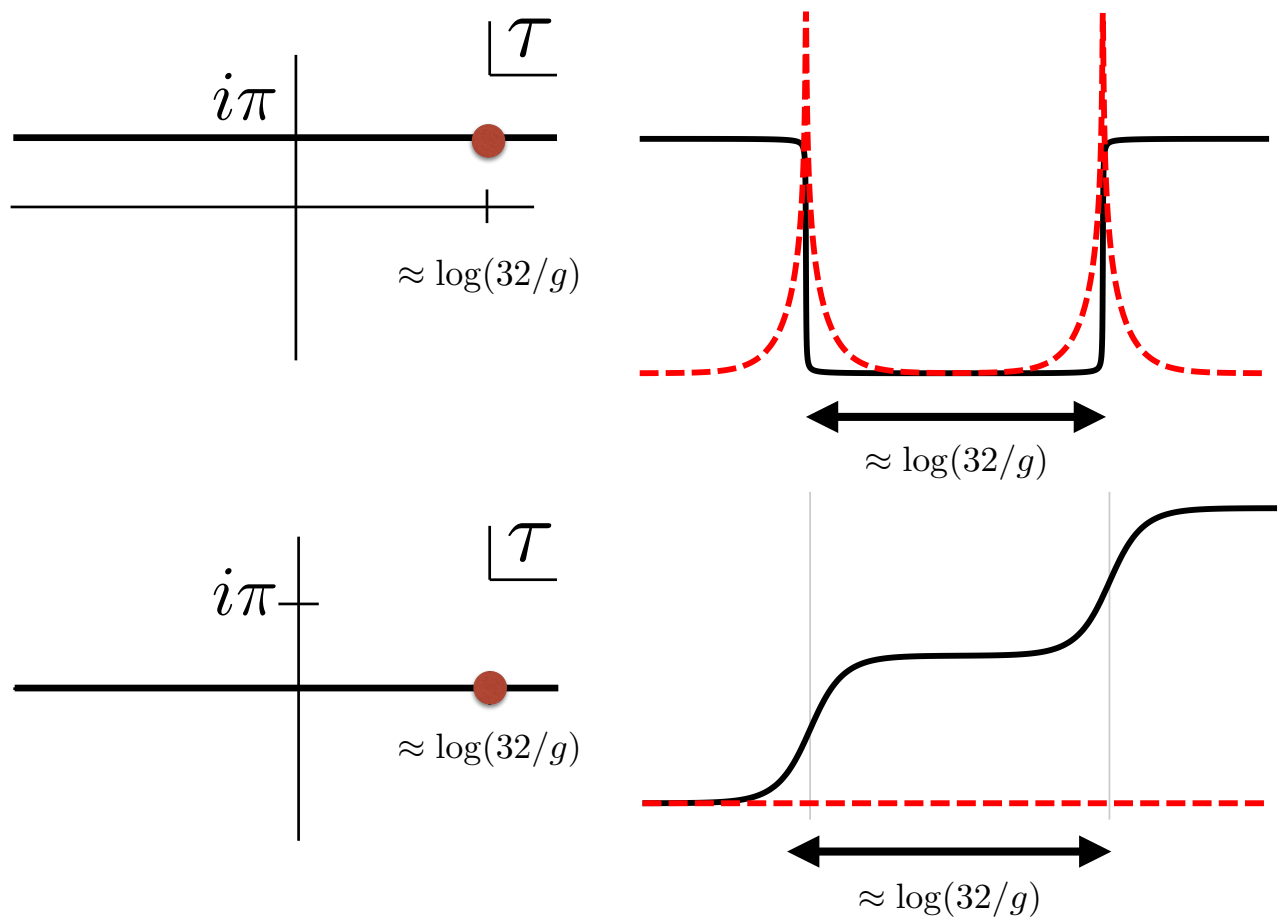

Figure 9. The quasi-zero mode (QZM) integral between two instanton events has a critical point at a finite (real) separation. The QZM integration for the instanton-anti-instanton has a a critical point at a finite (complex) separation. It is usually believed that two-events can at best be quasi-solutions due to non-linearity of the BPS equations. In this work, we prove that the figures on the right are actually exact non-BPS solutions to holomorphic Newton equations. We call these solutions complex (top) and real (bottom) bions. The figures are for $N_{f}=1$ and periodic potential.

The $[\mathcal{I I}]$ event, obtained by integrating over the QZM amplitude, has the form

$$
[\mathcal{I} \mathcal{I}]=I_{+}\left(N_{f}, g\right) \times[\mathcal{I}]^{2}=\left(\frac{g}{A a^{3}}\right)^{N_{f}} \Gamma\left(N_{f}\right) \times \frac{S_{I}}{2 \pi}\left[\frac{\operatorname{det}^{\prime} M}{\operatorname{det} M_{0}}\right]^{-1} e^{-2 S_{I}} .
$$

Meaning of the critical point at finite real separation and a puzzle: It is interesting to note that the critical point of the interaction between two instantons (4.1), unlike the bosonic case $N_{f}=0$ where it is located at infinity $m_{b} \tau^{*}=\infty$, is located at a finite real separation (4.3). This phenomena appeared first in field theory, in $\mathrm{QCD}(\mathrm{adj})$ for magnetic bions [1], and makes one suspect that there may be something "special" about such correlated two-events. The idea is that perhaps these correlated two-events are related to some exact solution with size $\tau^{*}=m_{b}^{-1} \ln \left(\frac{A a^{3}}{g N_{f}}\right)$. There is 
an apparent problem with this idea, however. Neither in the BPS-equation, nor in Newton's equation for the inverted bosonic potential, does the coupling constant $g$ or the number of fermions $N_{f}$ appear. But $m_{b} \tau^{*}$, the characteristic size of the correlatedtwo event depends on both $g$ and $N_{f}$. So, it seems impossible for these configurations to be solutions to the standard equations of motion in the inverted potential. We will solve this problem in this work, and show that the two-instanton correlated event is actually an approximation to an exact solution, that we refer to as a real bion, see Section 6.3, which solves the equation for a quantum modified potential.

\section{3 $[\mathcal{I} \overline{\mathcal{I}}]$ thimble integration and approximate form of complex bion}

The QZM integration for the $[\mathcal{I} \overline{\mathcal{I}}]$ correlated event is

$$
I_{-}\left(N_{f}, g\right)=\int_{\Gamma_{-}^{\mathrm{qzm}}} d\left(m_{b} \tau\right) e^{-\mathcal{V}_{-}(\tau)}=\int_{\Gamma_{-}^{\mathrm{qzm}}} d\left(m_{b} \tau\right) e^{-\left(-\frac{A a^{3}}{g} e^{-m_{b} \tau}+N_{f} m_{b} \tau\right)}
$$

where $\Gamma_{-}^{\mathrm{qzm}}$ is the QZM-cycle for the $[\mathcal{I} \overline{\mathcal{I}}]$ QZM-integration. The critical point of the integration is located at a complex point:

$$
\frac{d \mathcal{V}_{-}(\tau)}{d \tau}=0 \quad \tau^{*}=m_{b}^{-1}\left[\ln \left(\frac{A a^{3}}{g N_{f}}\right) \pm i \pi\right]= \begin{cases}\frac{1}{2 a}\left[\ln \left(\frac{16 a^{3}}{g N_{f}}\right) \pm i \pi\right] & (\mathrm{DW}) \\ \frac{1}{a}\left[\ln \left(\frac{32 a^{3}}{g N_{f}}\right) \pm i \pi\right] & (\mathrm{SG})\end{cases}
$$

The descent manifold associated with this critical point is in the complex plane, $\Gamma_{-}^{\mathrm{qzm}}=$ $\mathbb{R} \pm i \pi$, as can be deduced by solving the stationary phase condition (4.4): see Fig. 9. Substituting $u=e^{-m \tau}$, we map the integral to the standard representation of Gammafunction:

$$
I_{-}\left(N_{f}, g\right)=e^{ \pm i \pi N_{f}} \int_{0}^{\infty} d u u^{N_{f}-1} e^{-\frac{A a^{3}}{g} u}=e^{ \pm i \pi N_{f}}\left(\frac{g}{A a^{3}}\right)^{N_{f}} \Gamma\left(N_{f}\right) .
$$

The $[\mathcal{I} \overline{\mathcal{I}}]$ event, obtained by integrating over the QZM-thimble, has the form

$$
[\mathcal{I} \overline{\mathcal{I}}]_{ \pm}=I_{-}\left(N_{f}, g\right) \times[\mathcal{I}]^{2}=e^{ \pm i \pi N_{f}}\left(\frac{g}{A a^{3}}\right)^{N_{f}} \Gamma\left(N_{f}\right) \times \frac{S_{I}}{2 \pi}\left[\frac{\operatorname{det}^{\prime} M}{\operatorname{det} M_{0}}\right]^{-1} e^{-2 S_{I}}
$$

The integration over $\Gamma_{-}^{\mathrm{qzm}}$-thimbles is a rigorous version of the BZJ-prescription, and generalized to the multi-flavor theory.

Meaning of the critical point at finite complex separation, and another puzzle: For the instanton-anti-instanton, there is a critical point at a finite complex separation. All the concerns stated in the previous item stand. On top of that, apparently, 
the quasi-zero mode, a part of field space, is necessarily complexified in order to make sense of $[\mathcal{I} \overline{\mathcal{I}}]$. If we plot, for example, an instanton-anti-instanton separated by $\tau^{*}$, we see that the combination is actually complex, see the top part of Figure 9. Evidently, this cannot be a solution to the real Newton equation for the inverted bosonic potential. We will also solve this problem, and show that the instanton-anti-instanton event is an approximation to an exact complex solution, that we call complex bion, discussed in Sections 5.3 and 6.4, which solves the holomorphic Newton equation.

\subsection{Other aspects of thimble integration}

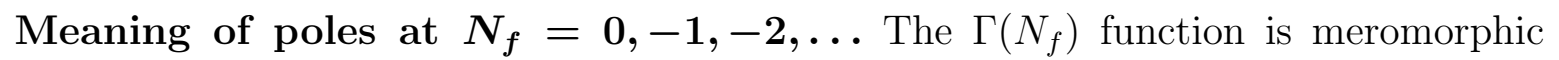
in the complexified $N_{f}$ plane, with poles at $N_{f}=0,-1,-2, \ldots$ if one analytically continues $N_{f} \in \mathbb{N}$ to complex numbers. Recall that a Hilbert space interpretation requires $N_{f} \in \mathbb{N}$, but as a statistical field theory, we are free to move to complex $N_{f}$ and view $N_{f}$ as a coupling constant (parameter) in the functional integral.

Thus, the correlated $[\mathcal{I} \mathcal{I}]$-amplitude is meromorphic in the whole complex $N_{f}$ plane except for the poles, which requires an interpretation. For $N_{f} \rightarrow 0^{+}$, the convergence factor due to fermion zero modes that regulates the large $\tau$ behavior disappears. The physical explanation of this phenomenon is that these events are uncorrelated, and are already included in the leading order semi-classical result. Expanding the result around $\epsilon=0$ we obtain

$$
I_{+}(\epsilon, g)=\left(\frac{g}{A a^{3}}\right)^{\epsilon} \Gamma(\epsilon)=\frac{1}{\epsilon}-\underbrace{\left(\ln \left(\frac{A a^{3}}{g}\right)+\gamma\right)}_{Q_{1}\left(N_{f}=0, g\right)}+O(\epsilon) .
$$

Subtracting off the $\frac{1}{\epsilon}$-pole in order to get rid of double counting of the uncorrelated two instanton events, we obtain the correlated two-event amplitude in the $N_{f}=0$ theory. The $N_{f}=0$ result is derived in [69] by using Lefschetz thimble of the bosonic theory. A similar subtraction is also needed for $N_{f}=-1,-2, \ldots$ as well. The result for the correlated 2-events for general $N_{f}$ is

$$
\begin{array}{ll}
{[\mathcal{I I}]=\left(\frac{g}{A a^{3}}\right)^{N_{f}} \Gamma\left(N_{f}\right) \frac{S_{I}}{2 \pi}\left[\frac{\operatorname{det}^{\prime} M}{\operatorname{det} M_{0}}\right]^{-1} e^{-2 S_{\mathcal{I}}},} & N_{f} \in \mathbb{C} \backslash\{0,-1,-2, \ldots\}, \\
{[\mathcal{I I}]=Q_{1}\left(N_{f}, g\right) \frac{S_{I}}{2 \pi}\left[\frac{\operatorname{det}^{\prime} M}{\operatorname{det} M_{0}}\right]^{-1} e^{-2 S_{\mathcal{I}}},} & N_{f}=0,-1,-2, \ldots
\end{array}
$$

For $N_{f}=0$, this results agrees with Bogomolny [28] and Zinn-Justin's bosonic result [29] and can also be obtained also via the WKB approximation. For $N_{f}=1$ this agrees with the result obtained with the use of supersymmetry [52, 67]. For small values of $N_{f}$ the polynomials $Q_{1}\left(N_{f}, g\right)$ are given by

$$
Q_{1}\left(N_{f}=0, g\right) \quad=-\gamma-\ln \left[A a^{3} / g\right]
$$




$$
\begin{aligned}
& Q_{1}\left(N_{f}=-1, g\right)=-1+\gamma+\ln \left[A a^{3} / g\right], \\
& Q_{1}\left(N_{f}=-2, g\right)=\frac{1}{4}\left(3-2 \gamma-2 \ln \left[A a^{3} / g\right]\right), \\
& Q_{1}\left(N_{f}=-3, g\right)=\frac{1}{36}\left(-11+6 \gamma+6 \ln \left[A a^{3} / g\right]\right) .
\end{aligned}
$$

Differentiating ambiguities from hidden topological angle: The $[\mathcal{I} \overline{\mathcal{I}}]$ amplitude can be written as

$$
\begin{array}{ll}
{[\mathcal{I} \overline{\mathcal{I}}]_{ \pm}=e^{ \pm i \pi N_{f}}\left(\frac{g}{A}\right)^{N_{f}} \Gamma\left(N_{f}\right) \frac{S_{I}}{2 \pi}\left[\frac{\operatorname{det}^{\prime} M}{\operatorname{det} M_{0}}\right]^{-1} e^{-2 S_{\mathcal{I}},}} & N_{f} \in \mathbb{C} \backslash\{0,-1,-2, \ldots\}, \\
{[\mathcal{I} \overline{\mathcal{I}}]_{ \pm}=Q_{1}\left(N_{f}, e^{ \pm i \pi} g\right) \frac{S_{I}}{2 \pi}\left[\frac{\operatorname{det}^{\prime} M}{\operatorname{det} M_{0}}\right]^{-1} e^{-2 S_{\mathcal{I}}},} & N_{f}=0,-1,-2, \ldots(4.14)
\end{array}
$$

where $Q_{1}\left(N_{f}, g\right)$ are the polynomials given in (4.13). This amplitude is divergent for $N_{f}=0,-1,-2, \ldots$. This divergence is the same as in the $[\mathcal{I I}]$ case, and is subtracted off to prevent double-counting.

The ambiguous imaginary part is related to resurgence. However, the ambiguous imaginary part disappears for $N_{f} \in \mathbb{N}^{+}$. This may imply the disappearance of the first would-be singularity in the Borel plane, located at $2 S_{I}$. Singularities at $4 S_{I}, 6 S_{I}, \ldots$ etc are still expected. This is to a certain degree similar to the disappearance of the first renormalon singularity in Borel plane in $\mathcal{N}=1 \mathrm{SYM}$ theory [70].

For $N_{f} \in \mathbb{N}^{+}$, there is a more subtle effect. $\operatorname{Re}[\mathcal{I} \overline{\mathcal{I}}] \propto e^{ \pm i \pi N_{f}}$ and this means that the contribution to the ground state energy due to $[\mathcal{I} \overline{\mathcal{I}}]$ is proportional to $(-1)^{N_{f}} \in \mathbb{Z}_{2}$, alternating in sign depending on $N_{f}$ being even or odd. Recall that $N_{f}$ even correspond to the integer spin, and $N_{f}$ odd correspond to the half integer spin. When we discuss exact solutions, we will provide an interpretation for this hidden topological angle in terms of topology in complexified field space.

\section{Graded formulation: Double-well potential}

Outline: In this section we construct new exact saddle solutions to the holomorphic Newton equations. In addition to the familiar real bounce solution, there is also a complex bion solution. The proliferation of the complex bions provides the Euclidean description of the ground state.

\subsection{Quantum modified potential and exact non-BPS solutions}

As described in Section 2.3, we grade the system with fermions according to fermion number, resulting in a sequence of quantum modified bosonic models, which we then complexify:

$$
\mathcal{L}(\dot{x}, x, \psi) \longrightarrow \bigoplus_{k=0}^{N_{f}} \operatorname{deg}\left(\mathcal{H}_{k}\right) \mathcal{L}_{k}(\dot{x}, x) \longrightarrow \bigoplus_{k=0}^{N_{f}} \operatorname{deg}\left(\mathcal{H}_{k}\right) \mathcal{L}_{k}(\dot{z}, z)
$$


The quantum modified (or graded) bosonic potentials are of the form

$$
V_{k}(z)=\underbrace{\frac{1}{2}\left(W^{\prime}(z)\right)^{2}}_{V_{\mathrm{bos}}(z)}+\underbrace{\frac{p g}{2} W^{\prime \prime}(z)}_{\text {fermion-induced }}, \quad p \equiv\left(2 k-N_{f}\right), \quad k=0, \ldots, N_{f} .
$$

For the case of the double-well we have $W^{\prime}(z)=z^{2}-a^{2}, W^{\prime \prime}=2 z$, so the fermioninduced potential introduces a tilting, breaking the degeneracy of the ground state of the bosonic potential. The non-perturbative normalization of the action makes it manifest that the fermion induced potential is $\mathcal{O}(g)$ with respect to bosonic potential $V_{\text {bos }}(z)$. Since $V_{\text {bos }}$ has exactly degenerate minima, an order $\mathcal{O}(g)$ tilting effect lifts the degeneracy of the classical potential, see Figure 10, and produces significant physical effects.

Recall that instantons satisfy a first order equation, $\dot{z}=W^{\prime}(z)$, already given in (3.4). Differentiating once with respect to time, the instanton also satisfies the second order equations of motion for a classical particle moving in the inverted potential, $-V_{\mathrm{bos}}=-\frac{1}{2}\left(W^{\prime}\right)^{2}$, namely

$$
\ddot{z}_{\text {instanton }}=\dot{z}_{\text {instanton }} W^{\prime \prime}=W^{\prime} W^{\prime \prime}=+\frac{\partial V_{\mathrm{bos}}}{\partial z} .
$$

Obviously, (3.4) and (5.3) do not involve $g$, the coupling constant, and neither does the solution. So, the correlated two-event discussed in Section 4, which has characteristic size $\tau^{*}=m_{b}^{-1} \ln \left(\frac{A a^{3}}{g N_{f}}\right)$ cannot be the solution to (3.4) and (5.3).

However, the saddle equations that one needs to solve are actually not these equations. Instead, we need to solve the complexified Newton equations for the inverted quantum modified potential:

$$
\ddot{z}_{\text {saddle }}=W^{\prime} W^{\prime \prime}+\frac{g p}{2} W^{\prime \prime \prime}=+\frac{\partial V_{\mathrm{bos}}}{\partial z}+\frac{g p}{2} W^{\prime \prime \prime} .
$$

This equation involves both $g$ and $p$ (i.e. spin projection), due to the fermion induced term, and we now show that it gives new exact solutions with characteristic size $\tau^{*}=$ $m_{b}^{-1} \ln \left(\frac{A a^{3}}{g N_{f}}\right)$.

Since the quantum modified potentials are quartic polynomials, the saddles are given in terms of standard elliptic functions, as follows. The saddles are found by using conservation of energy for a classical particle in the inverted potential. Consider a classical particle with Euclidean energy $E$ in potential $-V(z)$ :

$$
\begin{aligned}
& \mathcal{L}=\frac{1}{2} \dot{z}^{2}+V(z), \\
& E=\frac{1}{2} \dot{z}^{2}-V(z) .
\end{aligned}
$$




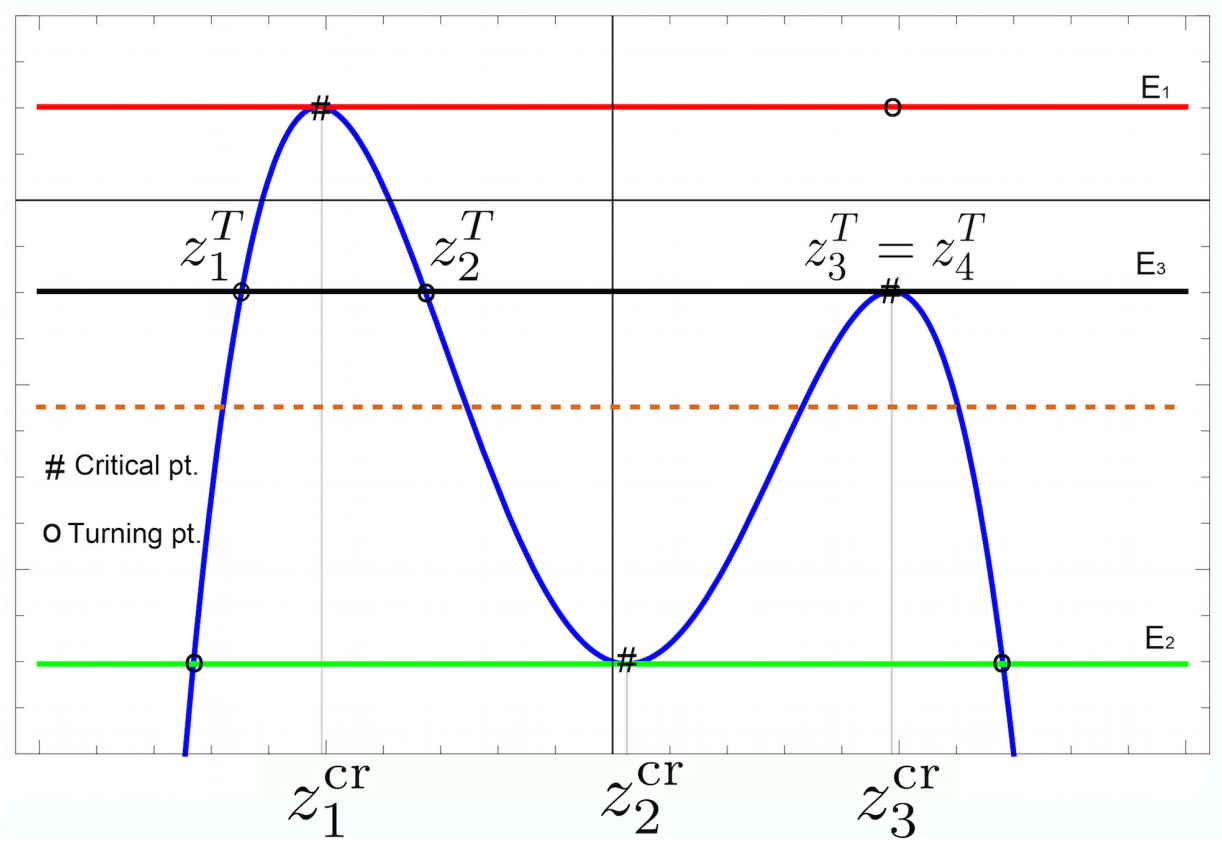

Figure 10. Inverted tilted DW potential. The three critical points are denoted $z_{a}^{\mathrm{cr}}, a=1,2,3$, and $E_{a}, a=1,2,3$ labels the Euclidean energy at these critical points.

For $p g / a^{3} \in \mathbb{R}$ and $p g \leq \frac{4 a^{3}}{3 \sqrt{3}}$, the potential has three real critical points, $z_{\ell}^{\text {cr }}, \ell=1,2,3$, whose expressions involve complicated cube roots. These can be usefully parametrized as follows:

$$
z_{\ell}^{\mathrm{cr}}=-\frac{2 a}{\sqrt{3}} \cos \left(\frac{1}{3} \arccos \left(\frac{3 \sqrt{3}}{4 a^{3}} p g\right)-\frac{2(\ell-1) \pi}{3}\right) \quad, \quad \ell=1,2,3
$$

For small $p g / a^{3} \ll 1$, the tilting is small and $z_{1}^{\mathrm{cr}} \approx-a, z_{2}^{\mathrm{cr}} \approx 0$, and $z_{3}^{\mathrm{cr}} \approx+a$ :

$$
\begin{aligned}
& z_{1}^{\mathrm{cr}} \approx-a-\frac{p g}{4 a^{2}}+\frac{3(p g)^{2}}{32 a^{5}}-O\left((p g)^{3}\right), \\
& z_{2}^{\mathrm{cr}} \approx \frac{p g}{2 a^{2}}+\frac{(p g)^{3}}{8 a^{8}}+O\left((p g)^{5}\right), \\
& z_{3}^{\mathrm{cr}} \approx a-\frac{p g}{4 a^{2}}-\frac{3(p g)^{2}}{32 a^{5}}-O\left((p g)^{3}\right) .
\end{aligned}
$$

The corresponding Euclidean energies, $E_{k} \equiv-V\left(z_{k}^{\mathrm{cr}}\right)$, also play an important role in the description of the exact saddle solutions. As shown in Figure 10, these Euclidean energies mark the boundaries at which turning points coalesce. These are points where two roots of the quartic $V(z)+E=0$ coalesce. For example, as $E$ approaches $E_{3}$ 
from below, the right-hand pair of real turning points coalesce, while for $E>E_{3}$, the turning points $z_{3}^{T}$ and $z_{4}^{T}$ become complex (they form a complex conjugate pair for $\left.p g / a^{3} \in \mathbb{R}\right)$. Similarly at $E=E_{1}$ the turning points $z_{1}^{T}$ and $z_{2}^{T}$ coalesce; they are real for $E_{2} \leq E \leq E_{1}$, but are complex for $E>E_{1}$.

It is useful to view the energy conservation relation as the projection of a complex algebraic curve to real momentum and position. Consider the complex algebraic curve:

$$
\mathcal{P}^{2}=2 V(z)+2 E=z^{4}-2 z^{2} a^{2}+2 g p z+a^{4}+2 E .
$$

This "energy conservation" (5.8) can be integrated by quadratures to give:

$$
\int_{t_{0}=0}^{t} d t=\int_{z_{T}}^{z} \frac{d z}{\mathcal{P}}=\int_{z_{T}}^{z} \frac{d z}{\sqrt{2(E+V(z))}} .
$$

This integral can be computed exactly in terms of the Weierstrass $\wp$-function, and takes a particularly simple form when $z_{T}$ is a turning point; i.e., if $z_{T}$ is any root of the equation $E+V(z)=0$ (see Appendix A). The solution is

$$
z(t)=z_{T}+\frac{\frac{1}{2} V^{\prime}\left(z_{T}\right)}{\wp\left(t ; g_{2}, g_{3}\right)-\frac{1}{12} V^{\prime \prime}\left(z_{T}\right)} .
$$

Here, the algebraic invariants $g_{2}(E)$ and $g_{3}(E)$ are given by

$$
\begin{aligned}
& g_{2}(E)=2 E+\frac{4 a^{4}}{3}, \\
& g_{3}(E)=-\frac{8 a^{6}}{27}-\frac{2 E a^{2}}{3}-\frac{p^{2} g^{2}}{4} .
\end{aligned}
$$

The exact solution (5.10) has a center position modulus, associated with translation symmetry in $t$, which can be restored by setting $t \rightarrow t-t_{\mathrm{c}}$. Without loss of generality, we set $t_{\mathrm{c}}=0$ from now on.

Since the classical saddle bounce and bion solutions begin and end on turning points (either real or complex), they can all be expressed in closed form using the expression (5.10). All that needs to be done is to match to the appropriate boundary conditions.

The Weierstrass $\wp$-function is doubly periodic, with a real and complex (in our case, purely imaginary) period ${ }^{13}, T_{1}$ and $T_{2}$. There is a simple interpretation of the double periodicity. The real period is the period of bounded motion in the potential $-V(z)$ with energy $E$. To understand the imaginary period, take $t \rightarrow i t$ (back to Minkowski space for a moment) and observe that this reverses the signs of potential

\footnotetext{
${ }^{13}$ Standard mathematical notation for the two periods are $\left(2 \omega_{1}, 2 \omega_{2}\right)$, which we call here $\left(T_{1}, T_{2}\right)$. See, for example, Whittaker and Watson [71].
} 


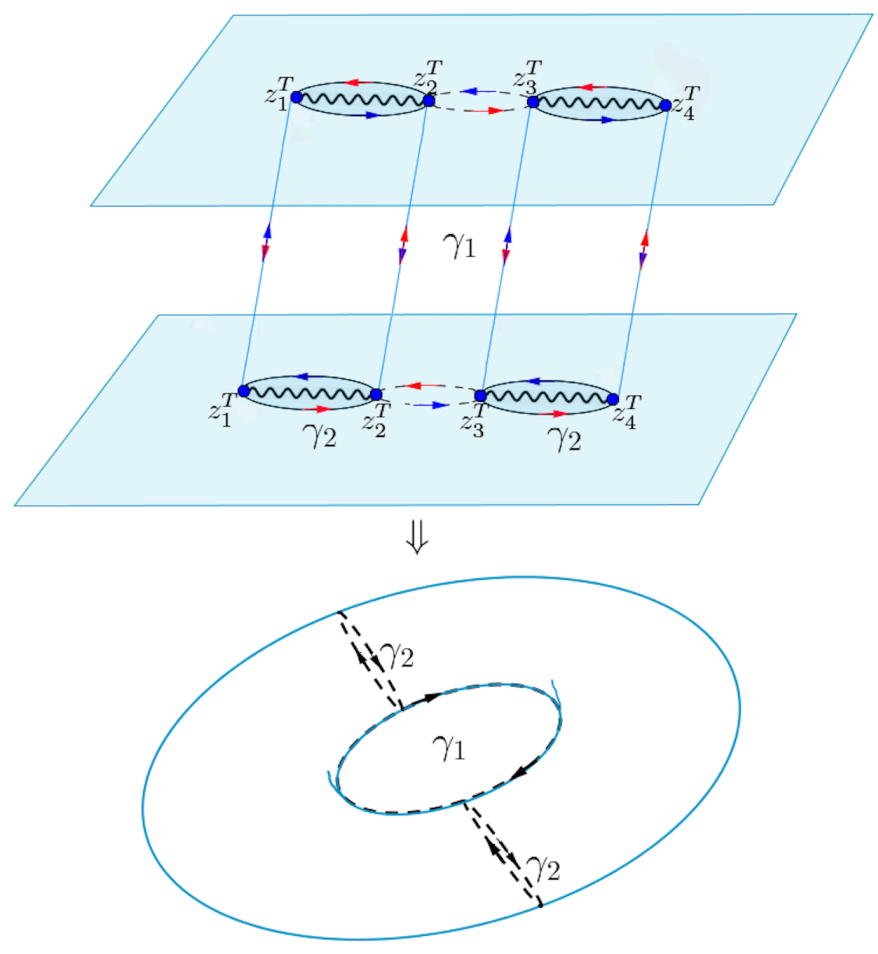

Figure 11. Doubly periodic structure at a typical energy $E$. At critical energies shown in Fig.10, two of the turning points degenerates.

and energy in the conservation of energy: $(-V(z), E) \rightarrow(V(z),-E)$. The magnitude of the imaginary period is the period of classical motion either on the left or right well. Note that the left-well and right-well periods are actually equal to each other, for any asymmetric double-well quartic potential! This remarkable fact helps us to write down simpler expressions for exact solutions. Explicit expressions for the two periods are (see Figure 11).

$$
\begin{aligned}
& T_{1}(E)=\int_{\gamma_{1}} \frac{d z}{\mathcal{P}}=2 \int_{z_{2}(E)}^{z_{3}(E)} \frac{d z}{\mathcal{P}} \\
& T_{2}(E)=\int_{\gamma_{2}} \frac{d z}{\mathcal{P}}=2 \int_{z_{1}(E)}^{z_{2}(E)} \frac{d z}{\mathcal{P}}=2 \int_{z_{3}(E)}^{z_{4}(E)} \frac{d z}{\mathcal{P}} .
\end{aligned}
$$

where $\gamma_{1,2}$ are the cycles defined on a Riemann torus

\subsection{Exact (real) bounce solution}

The bounce solution is a solution with Euclidean energy $E=E_{3}$, equal to minus the potential at the lower of the maxima of $-V(z)$, see Figure 10. At this energy there are 
three real turning points. It is useful to parametrize all the turning points (which are cumbersome in terms of original parameters of the potential), as well as exact solutions in terms of a critical point. This provides relatively simple expressions:

$$
\left\{z_{1}^{T}, z_{2}^{T}, z_{3}^{T}=z_{4}^{T}\right\}=\left\{-z_{3}^{\mathrm{cr}}-\sqrt{\frac{p g}{z_{3}^{\mathrm{cr}}}},-z_{3}^{\mathrm{cr}}+\sqrt{\frac{p g}{z_{3}^{\mathrm{cr}}}}, z_{3}^{\mathrm{cr}}\right\} .
$$

The "bounce" solution [5] bounces off the (real) turning point $z_{T}=-z_{3}^{\mathrm{cr}}+\sqrt{\frac{p g}{z_{3}^{\mathrm{cr}}}}$ at some finite time $t=0$, tending as $t \rightarrow \pm \infty$ to the lower of the (real) maxima of the inverted potential, $z=z_{3}^{\mathrm{cr}}$. At this particular energy, $T_{1}$ diverges and the doubly-periodic Weierstrass function in (5.10) degenerates to a singly-periodic function:

$$
\wp(t)=\frac{\omega_{\mathrm{bn}}^{2}}{4}\left(\frac{1}{\sinh ^{2}\left(\omega_{\mathrm{bn}} t / 2\right)}+\frac{1}{3}\right)
$$

where

$$
\omega_{\mathrm{bn}}=\sqrt{V^{\prime \prime}\left(z_{3}^{\mathrm{cr}}\right)}
$$

is the curvature of the potential at $z_{3}^{\text {cr }} \cdot{ }^{14}$ This corresponds to the singly-periodic limit where $\omega_{\mathrm{bn}}^{2}=-18 g_{3}\left(E_{3}\right) / g_{2}\left(E_{3}\right)$, in terms of the algebraic lattice invariants $g_{2}(E)$ and $g_{3}(E)$ in (A.6).

Thus, the exact bounce solution can be written as (here $\left.z_{T}=-z_{3}^{\mathrm{cr}}+\sqrt{\frac{p g}{z_{3}^{\mathrm{cr}}}}\right)$

$$
\begin{aligned}
z_{\mathrm{bn}}(t) & =z_{3}^{\mathrm{cr}}+\left(z_{T}-z_{3}^{\mathrm{cr}}\right) \frac{\cosh ^{2}\left(\omega_{\mathrm{bn}} t_{0} / 2\right)}{\cosh ^{2}\left(\omega_{\mathrm{bn}} t / 2\right)+\sinh ^{2}\left(\omega_{\mathrm{bn}} t_{0} / 2\right)} \\
& =z_{T}+\left(z_{3}^{\mathrm{cr}}-z_{T}\right) \frac{\sinh ^{2}\left(\omega_{\mathrm{bn}} t / 2\right)}{\sinh ^{2}\left(\omega_{\mathrm{bn}} t / 2\right)+\cosh ^{2}\left(\omega_{\mathrm{bn}} t_{0} / 2\right)}
\end{aligned}
$$

where (see Appendix A)

$$
t_{0}=\frac{2}{\omega_{\mathrm{bn}}} \operatorname{arccosh}\left(\sqrt{\frac{3}{1-V^{\prime \prime}\left(z_{T}\right) / \omega_{\mathrm{bn}}^{2}}}\right) .
$$

${ }^{14}$ The linear frequency on the right $(\mathrm{R})$ well at $z=z_{3}^{\mathrm{cr}}$ (the value of the frequency as one take $E \rightarrow-E_{3}$ limit in the original potential) is equal to the non-linear frequency on the left (L) well at $z=z_{1}^{\text {cr }}$ for the energy level $E=-E_{3}$ !

$$
\omega_{\mathrm{bn}}=\omega_{\text {linear }}^{\mathrm{R}}\left(E=-E_{3}\right)=\omega_{\text {non-linear }}^{\mathrm{L}}\left(E=-E_{3}\right) .
$$

This identity is a consequence of the relation (5.12) for the periods of the complex algebraic curve. Physically, in the singly periodic limit of the Weierstrass function, $i \omega_{\text {non-linear }}^{\mathrm{L}}\left(E=-E_{3}\right)$ is the (pure imaginary) frequency which remains finite, while the other real frequency tends to zero. 


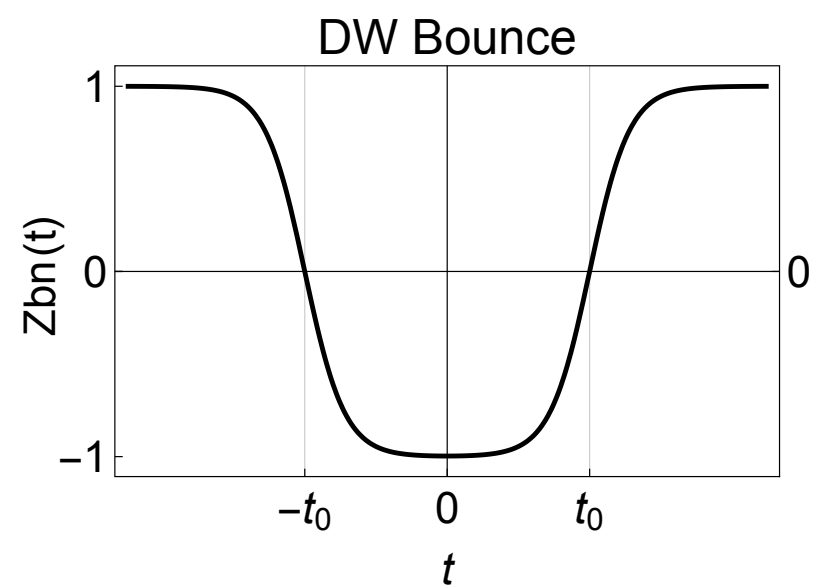

Figure 12. Bounce solution in the double well case. Note that the separation between instanton-anti-instanton is $2 t_{0} \approx \frac{1}{2 a} \ln \left(\frac{16 a^{3}}{p g}\right)$.

Comparing with (A.8), we see that this solution satisfies the desired boundary conditions:

$$
z_{\mathrm{bn}}( \pm \infty)=z_{3}^{\mathrm{cr}}, \quad z_{\mathrm{bn}}(0)=z_{T} \equiv-z_{3}^{\mathrm{cr}}+\sqrt{\frac{p g}{z_{3}^{\mathrm{cr}}}}
$$

The bounce solution is shown in Figure 12 .

It is physically more intuitive to rewrite the exact bounce solution (5.18) as

$$
z_{\mathrm{bn}}(t)=z_{3}^{\mathrm{cr}}-\frac{\left(z_{3}^{\mathrm{cr}}-z_{T}\right)}{2} \operatorname{coth}\left(\omega_{\mathrm{bn}} \frac{t_{0}}{2}\right)\left(\tanh \left[\omega_{\mathrm{bn}} \frac{\left(t+t_{0}\right)}{2}\right]-\tanh \left[\omega_{\mathrm{bn}} \frac{\left(t-t_{0}\right)}{2}\right]\right)
$$

where $t_{0}$ is given by (5.19). This expression makes it clear that the real bounce solution has a kink/anti-kink form. In fact, the exact bounce solution is nothing but the exact version of the approximate correlated anti-instanton/instanton $[\overline{\mathcal{I}} \mathcal{I}]$ event in the language of Section 4 . The bounce is an $\overline{\mathcal{I}}$ event taking place at $t=-t_{0}$, followed by an instanton event $\mathcal{I}$ at $t=+t_{0}$. The separation between these two events is fixed and is given by

$$
2 t_{0} \approx m_{b}^{-1} \ln \frac{16 a^{3}}{p g} \equiv \tau^{*} \quad, \quad \text { for } g p \ll a^{3} .
$$

This is precisely the scale which dominates the quasi-zero-mode thimble integration: $\tau^{*}=m_{\mathrm{b}}^{-1} \ln \left(\frac{A a^{3}}{g N_{f}}\right)$, where in this case $A=16, m_{b}=2 a$, and $p=N_{f}$ in (4.3).

An important characteristic property of the solution is the parametrically large plateau, i.e, parametrically large time spent around the turning point $z_{T}$. This makes 
this solution slightly different from the conventional bounce solutions (which looks just like a regular bump). Therefore a more appropriate name would be a flat bounce. The classical particle starts at the local maximum $z_{3}^{\text {cr }}$ at $t=-\infty$ (see Fig. 10). After spending an infinitely long time there, it rolls down at an instant (like an instanton), and reaches the vicinity of the turning point $z_{2}^{T}$ within a time scale $\sim a^{-1}$, characteristic of instantons. Since $z_{2}^{T}$ is is parametrically close to the global maximum, the classical particle spends a large time span $2 t_{0} \sim m_{b}^{-1} \ln \frac{16 a^{3}}{g p}$ near the turning point $z_{2}^{T}$. The Euclidean particle gets parametrically close to the global maximum $z_{1}^{\text {cr }},\left|z_{3}^{\text {cr }}-z_{2}^{T}\right| \sim$ $\sqrt{p g / a}$, and returns to the local maximum $z_{3}^{\text {cr }}$. The crucial point is that the size of the flat-bounce solution is not controlled by the inverse natural frequency of the system $\sim(2 a)^{-1} \equiv m_{b}^{-1}$, which is the case for conventional instantons as well as bounce solutions. Rather, the size of the flat-bounce solution is parametrically larger than the instanton size, by a factor $\ln \frac{16 a^{3}}{g p}$. For a harmonic unstable equilibrium point, a particle with energy $E \rightarrow E_{1}$ from below will spend a time span of $\log \left|E-E_{1}\right|$ in the neighborhood of the critical point. This form of divergence is universal around the critical point. In the present case, for $E=E_{2}$, the time span on the plateau is $-\log \left|E_{2}-E_{1}\right|=\log \frac{16 a^{3}}{p g}$. This feature leads to some interesting new effects when we discuss the fluctuation operators around these exact new saddles in Section 6.9.

\subsection{Exact complex bion solution}

The complex bion solution is a complex classical solution with Euclidean energy $E=$ $E_{1}$, equal to minus the potential evaluated at the higher of the maxima of $-V(z)$. See Figure 10. The complex bion bounces off one of the complex turning points $z=$ $z_{T}=-z_{1}^{\mathrm{cr}}+i \sqrt{\frac{p g}{-z_{1}^{\mathrm{cr}}}}$, (or its complex conjugate $z=z_{T}^{*}$ ), at some finite time $t=0$, and reaches the upper of the (real) maxima of the inverted potential, $z=z_{1}^{\text {cr }}$ as $t \rightarrow \pm \infty$, see Figure 10. The doubly-periodic Weierstrass function in (5.10) degenerates again to a singly-periodic function, and we obtain a solution of the same form as the bounce solution in (5.18), but with different critical point and turning point:

$$
\begin{aligned}
z_{\mathrm{cb}}(t) & =z_{1}^{\mathrm{cr}}+\left(z_{T}-z_{1}^{\mathrm{cr}}\right) \frac{\cosh ^{2}\left(\omega_{\mathrm{cb}} t_{0} / 2\right)}{\cosh ^{2}\left(\omega_{\mathrm{cb}} t / 2\right)+\sinh ^{2}\left(\omega_{\mathrm{cb}} t_{0} / 2\right)} \\
& =z_{T}+\left(z_{1}^{\mathrm{cr}}-z_{T}\right) \frac{\sinh ^{2}\left(\omega_{\mathrm{cb}} t / 2\right)}{\sinh ^{2}\left(\omega_{\mathrm{cb}} t / 2\right)+\cosh ^{2}\left(\omega_{\mathrm{cb}} t_{0} / 2\right)},
\end{aligned}
$$

where $z_{T}=-z_{1}^{\mathrm{cr}}+i \sqrt{\frac{p g}{-z_{1}^{\mathrm{cr}}}}$, and

$$
\omega_{\mathrm{cb}}=\sqrt{V^{\prime \prime}\left(z_{1}^{\mathrm{cr}}\right)}
$$




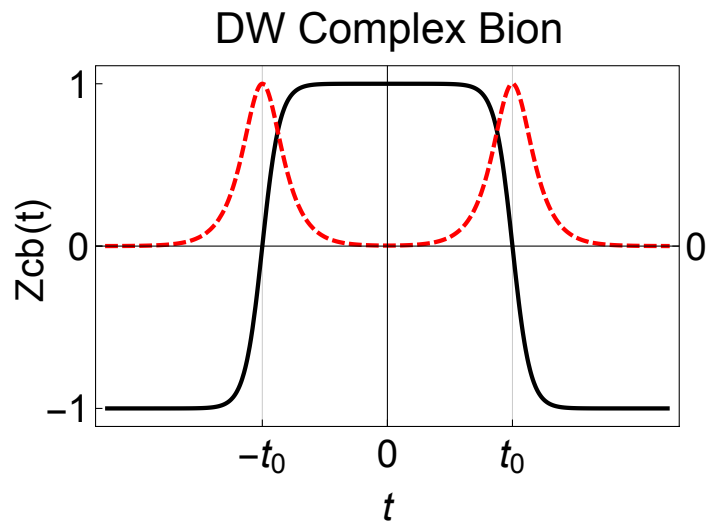

Figure 13. Real (black) and imaginary (red) parts of the complex bion for the double well case. The real part of the separation is $\operatorname{Re}\left(2 t_{0}\right) \approx \frac{1}{2 a} \ln \left(\frac{16 a^{3}}{p g}\right)$.

The parameter $t_{0}$ is given by (see Appendix A)

$$
t_{0}=\frac{2}{\omega_{\mathrm{cb}}} \operatorname{arccosh}\left(\sqrt{\frac{3}{1-V^{\prime \prime}\left(z_{T}\right) / \omega_{\mathrm{cb}}^{2}}}\right) .
$$

Comparing with (A.8), we see that this solution satisfies the desired boundary conditions:

$$
z_{\mathrm{cb}}( \pm \infty)=z_{1}^{\mathrm{cr}}, \quad z_{\mathrm{cb}}(0)=z_{T}=-z_{1}^{\mathrm{cr}}+i \sqrt{\frac{p g}{-z_{1}^{\mathrm{cr}}}} .
$$

We can also write the complex bion as

$$
z_{\mathrm{cb}}(t)=z_{1}^{\mathrm{cr}}-\frac{\left(z_{1}^{\mathrm{cr}}-z_{T}\right)}{2} \operatorname{coth}\left(\frac{\omega_{\mathrm{cb}} t_{0}}{2}\right)\left(\tanh \left[\omega_{\mathrm{cb}} \frac{\left(t+t_{0}\right)}{2}\right]-\tanh \left[\omega_{\mathrm{cb}} \frac{\left(t-t_{0}\right)}{2}\right]\right),
$$

where $t_{0}$ is given by (5.26). Notice that $\omega_{\mathrm{cb}}$ is real, but $t_{0}$ is complex, as is $z_{T}$. Thus, the complex bion has both real and imaginary parts. The complex bion is plotted in Figure 13.

At weak coupling, we see that an approximate form of the exact solution (5.28) is directly related to the correlated instanton-anti-instanton $[\mathcal{I} \overline{\mathcal{I}}]$ pair discussed in Section 4. To see this we can use the fact that, in the weak coupling regime, the complex turning point, $z_{T}$, and the critical point $z_{1}^{\text {cr }}$ have the expansions:

$$
\begin{array}{ll}
z_{T}=a+i \sqrt{\frac{p g}{a}}+\frac{p g}{4 a^{2}}+\ldots, & z_{1}^{\mathrm{cr}}=-a-\frac{p g}{4 a^{2}}+\ldots, \\
V^{\prime \prime}\left(z_{T}\right)=(2 a)^{2}+12 i \sqrt{a p g}+3 p g / a+\ldots, & V^{\prime \prime}\left(z_{1}^{\mathrm{cr}}\right)=\omega_{\mathrm{cb}}^{2}=(2 a)^{2}+\frac{3 p g}{a}+\ldots
\end{array}
$$


The complex turning points in the bion solutions acquire an elegant reinterpretation in terms of the complex quasi-zero mode in the instanton-anti-instanton correlated event. To see this, consider the kink/anti-kink form in (5.28). The separation between the instanton and anti-instanton (5.26) has both real and imaginary parts. Using the weak coupling expansions given in (5.29), the approximate form of the complex separation can be written as

$$
2 t_{0 c}^{ \pm} \approx m_{b}^{-1}\left[\ln \left(\frac{16 a^{3}}{p g}\right) \pm i \pi\right] \approx \tau^{*}
$$

This is precisely the scale which dominates the quasi-zero-mode thimble integration: $\tau^{*}=m_{\mathrm{b}}^{-1} \ln \left(\frac{A a^{3}}{g N_{f}}\right)$, where in this case $A=16, \omega_{\mathrm{cb}} \approx m_{b}=2 a$, and $p=N_{f}$ in (4.8). This proves that the integration over the complexified quasi-zero mode described in Section 4 produces the most significant physical features of the exact complex bion solution, such as the size of the two-event and most importantly, the hidden topological angle.

\subsection{Actions and amplitudes of the (real) bounce and the complex bion}

The bounce and complex bion solutions have finite Euclidean action. To obtain the action we simply integrate

$$
g S=2 \int_{z_{\mathrm{cr}}}^{z_{T}} d z \sqrt{2 E+2 V(z)},
$$

where $z_{\text {cr }}$ is the critical point, corresponding to the initial and final point of the solution, and $z_{T}$ is the turning point. For the bounce, the critical point is $z_{3}^{\mathrm{cr}}$ associated with the energy level $E_{3}$ in Fig. 10 and for the complex bion, the critical point is $z_{1}^{\text {cr }}$ associated with the energy level $E_{1}$ in Fig. 10:

$$
\begin{array}{cc}
\text { bounce }(\mathrm{bn}): z_{\mathrm{cr}}=z_{3}^{\mathrm{cr}}, & z_{T}=-z_{3}^{\mathrm{cr}}+\sqrt{\frac{p g}{z_{3}^{\mathrm{cr}}}} \\
\text { complex bion }(\mathrm{cb}): z_{\mathrm{cr}}=z_{1}^{\mathrm{cr}}, & z_{T}=-z_{1}^{\mathrm{cr}}+i \sqrt{\frac{p g}{-z_{1}^{\mathrm{cr}}}} .
\end{array}
$$

Note that the turning point $z_{T}$ is real in the case of the bounce, and complex in the case of the complex bion. Both $z_{1}^{\text {cr }}$ and $z_{3}^{\text {cr }}$ are real. To evaluate (5.31), since either $E_{1}$ level and $E_{3}$ level are associated with the separatrices (critical orbits) where two of the roots of the quadratic polynomial degenerate, we can express the integrand as

$$
2 E+2 V(z)=\left(z-z_{\mathrm{cr}}\right)^{2}\left(z-z_{T}\right)\left(z-z_{T}^{\prime}\right)
$$


where $z_{T}^{\prime}$ is the other turning point. Then the action integral (5.31) can be performed and it yields

$$
\begin{aligned}
& g S=\frac{1}{12}\left\{\sqrt{\left(z_{\mathrm{cr}}-z_{T}\right)\left(z_{\mathrm{cr}}-z_{T}^{\prime}\right)}\left[3\left(z_{T}-z_{T}^{\prime}\right)^{2}+4\left(z_{\mathrm{cr}}-z_{T}\right)\left(z_{\mathrm{cr}}-z_{T}^{\prime}\right)\right]\right. \\
&\left.\quad-3\left(z_{T}-z_{T}^{\prime}\right)^{2}\left(2 z_{\mathrm{cr}}-z_{T}-z_{T}^{\prime}\right) \ln \left(\sqrt{\frac{z_{\mathrm{cr}}-z_{T}}{z_{T}-z_{T}^{\prime}}}+\sqrt{\frac{z_{\mathrm{cr}}-z_{T}^{\prime}}{z_{T}-z_{T}^{\prime}}}\right)\right\} .
\end{aligned}
$$

This expression can be simplified significantly using simple algebraic properties of the critical points and turning points. Equating (5.8) with (5.33) at the critical energy levels, the vanishing of the cubic term implies: $2 z_{\mathrm{cr}}+z_{T}+z_{T}^{\prime}=0$. Comparing the linear terms in the two expressions, we obtain $-\left(z_{T}+z_{T}^{\prime}\right) z_{\mathrm{cr}}^{2}-2 z_{T} z_{T}^{\prime} z_{\mathrm{cr}}=2 p g$. These two equations allow us to express both $z_{T}$ and $z_{T}^{\prime}$ in terms of $z_{\mathrm{cr}}$,

$$
z_{T}=-z_{\mathrm{cr}}+\sqrt{\frac{p g}{z_{\mathrm{cr}}}}, \quad \quad z_{T}^{\prime}=-z_{\mathrm{cr}}-\sqrt{\frac{p g}{z_{\mathrm{cr}}}} .
$$

which is a useful parametrization. Finally, comparing quadratic terms, one obtains

$$
\frac{2 z_{\mathrm{cr}}^{3}+p g}{z_{\mathrm{cr}}}=2 a^{2}
$$

This equation has three solutions. These are just the three critical points in (5.6) and (5.7). For the bounce we choose $z_{\mathrm{cr}}=z_{3}^{\mathrm{cr}}$, and for the complex bion we choose $z_{\mathrm{cr}}=z_{1}^{\mathrm{cr}}$. Using these relations, the action (5.34) reduces to

$$
\begin{aligned}
S_{\text {saddle }} & =\frac{4 a^{2} \sqrt{6 z_{\mathrm{cr}}^{2}-2 a^{2}}}{3 g}-4 p \ln \left(\sqrt{\frac{1}{2}+\sqrt{z_{\mathrm{cr}}^{3} / p g}}+\sqrt{-\frac{1}{2}+\sqrt{z_{\mathrm{cr}}^{3} / p g}}\right) \\
& =\frac{8 a^{3}}{3 g} \sqrt{1-\frac{3 p g}{4 a^{2} z_{\mathrm{cr}}}}+2 p \operatorname{arccosh}\left(2 \sqrt{\frac{1}{2}+\frac{z_{\mathrm{cr}}^{3}}{p g}}\right) .
\end{aligned}
$$

For the bounce we choose $z_{\mathrm{cr}}=z_{3}^{\mathrm{cr}}, S_{\text {saddle }}$ is real, and for the complex bion we choose $z_{\mathrm{cr}}=z_{1}^{\mathrm{cr}}, S_{\mathrm{cb}}$ has both real and imaginary parts. In particular, it is easy to write that, by simply observing the fact that $z_{1}^{\text {cr }}<0$, and expressing (5.37a) in terms of real and imaginary parts,

$$
S_{\mathrm{cb}}=\operatorname{Re}\left[S_{\mathrm{cb}}\right] \pm i p \pi, \quad \operatorname{Im}\left[S_{\mathrm{cb}}\right]= \pm p \pi
$$

The imaginary part is the hidden topological angle (HTA) discussed in [26] for integer $p$. For non-integer $p$, it is related both to HTA and resurgent cancellations with perturbation theory. The sign of the HTA depends on the choice of complex turning point, 
see Figure 14. A crucial point is that the imaginary part is independent of $p g / a^{3}$ (so long $p g / a^{3}$ is small enough not to alter the tilted double-well structure.)

In fact, it is more illuminating to express the actions in the weak coupling regime,

$\frac{p g}{a^{3}} \ll 1$. For the (real) bounce solution, $S_{\text {saddle }}$ is real, while for the complex bion $S_{\text {saddle }}$ has an imaginary part:

$$
\begin{aligned}
& S_{\mathrm{bn}}=\frac{8 a^{3}}{3 g}+p \ln \left(\frac{p g}{16 a^{3}}\right)+\mathcal{O}\left(g^{1 / 2}\right), \\
& S_{\mathrm{cb}}=\frac{8 a^{3}}{3 g}-p \ln \left(\frac{p g}{16 a^{3}}\right) \pm i \pi p+\mathcal{O}\left(g^{1 / 2}\right) .
\end{aligned}
$$

Clearly, the real part of the action of the complex bion is larger than twice the instanton action in the original formulation, which, in turn is larger than the action of the bounce.

These weak coupling expressions are in precise agreement with the original formulation in Section 4, where we obtained instanton-anti-instanton correlated two-events by integration over the quasi-zero mode $(\mathrm{QZM})$ thimble, $\Gamma^{\mathrm{qzm}}$. The corresponding amplitude of the complex bion and real bounce take the form:

$$
\begin{aligned}
I_{\mathrm{bn}} & \sim\left(\frac{p g}{16 a^{3}}\right)^{-p} e^{-2 S_{I}}, \\
I_{\mathrm{cb}} & \sim\left(\frac{p g}{16 a^{3}}\right)^{p} e^{-2 S_{I} \pm i p \pi},
\end{aligned}
$$

as can be deduced in the original formulation. This one-to-one correspondence between the exact complex and real solutions and the complexification of the (QZM)-thimbles suggests that the latter rationale is actually a systematic approximation to the saddles in the full quantum theory.

\subsection{Analytic continuation and monodromy: From real bounce to complex bion}

By the discussion of Section 2.7, we know that for the tilted double-well potential, $P \widehat{H}_{+p} P=\widehat{H}_{-p}$, namely parity maps one Hamiltonian system to an isospectral Hamiltonian system, by (2.37). The non-perturbative effects in the paired graded systems are same, and can be obtained from each other by analytic continuation. In particular, we can obtain the complex bion solution by analytic continuation of the real bounce solution, essentially analytically continuing $p \rightarrow-p$. Consider analytic continuation of the tilted DW theory to the complex $p$ plane, namely:

$$
p \rightarrow p e^{i \theta} \in \mathbb{C}
$$

Start with $V_{+}(z)=\frac{1}{2}\left(W^{\prime}(z)\right)^{2}+\frac{p g}{2} W^{\prime \prime}(z)$. Turn on $\theta \neq 0$. At $\theta=\pi$, we reach the theory described by the potential $V_{-}(z)$, which is the parity transform of $V_{+}(z)$. 
$\theta=0$
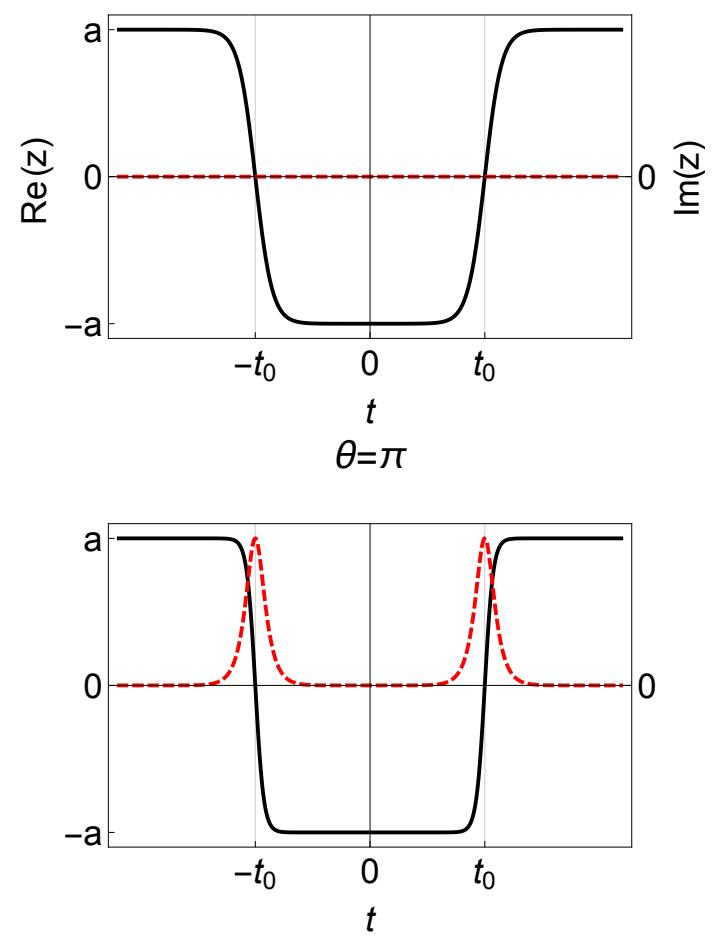

$\theta=\pi / 2$
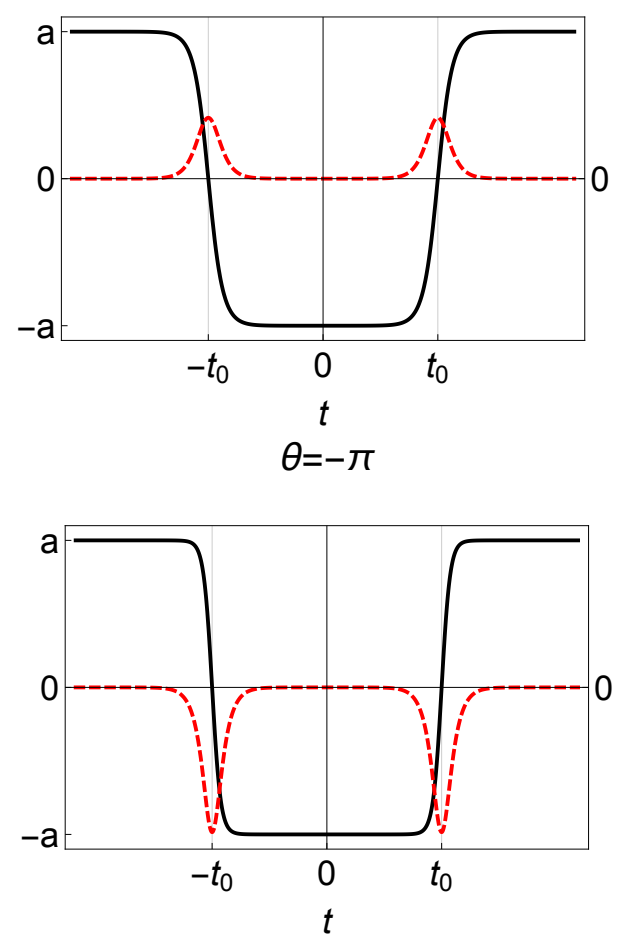

Figure 14. This figure shows the real (solid black line) and imaginary (dashed red line) parts of the classical solution $z(t)$ in the double well potential for $p g / a^{3} \ll 1$, and a range of values of the $\theta$ parameter. The $\theta=0$ solution corresponds to the real bounce, $\theta=\pi$ and $\theta=-\pi$ are the complex bions. The solution has monodromy $4 \pi$, or order 2 monodromy.

Upon analytic continuation all the way to $\theta=\pi$ : (i) The local maximum (of course, restricted to the real axis) of $-V_{+}(z)$ becomes the global maximum of $-V_{-}(z)$. (ii) The global maximum of the $-V_{+}(z)$ becomes the local maximum of $-V_{-}(z)$. (iii) The real turning point of the real bounce solution in the $-V_{+}(z)$ system turns into a complex turning point in the $-V_{-}(z)$ system. At intermediate $\theta$, the complex solution is always a complex classical solution to the holomorphic equations of motions, and the profiles of the real and imaginary parts are shown in Figure 14 and Figure 15 for various values of $\theta$.

Fig. 16 depicts the real and imaginary part of the action $(\operatorname{Re}(S), \operatorname{Im}(S))$ of the analytically continued real bounce solution as a function of $\theta \cdot \theta=0$ correspond to the real bounce solution, which is purely real. Note that the action of the real bounce is less than two-times instanton action, $2 S_{I}$, in terms of the instanton action of the original formulation. The reason for this is that the bounce is associated with the 


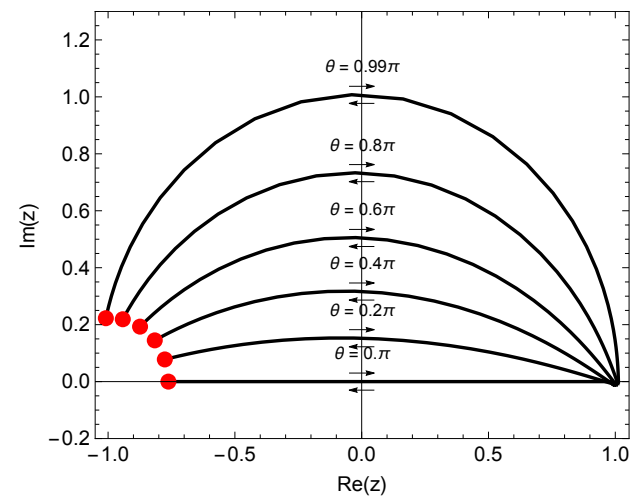

Figure 15. Parametric plot of the real and imaginary parts of the analytic continuation of the real bounce solution $z(t)$. We continue $p \rightarrow p e^{i \theta}$ and show the solution for a range of $\theta$ parameters. $\theta=0$ corresponds to the real bounce solution, with a real turning point. As one changes $\theta$, the turning point becomes complex. Exactly at $\theta=\pi^{-}$, the real bounce turns into a complex bion.

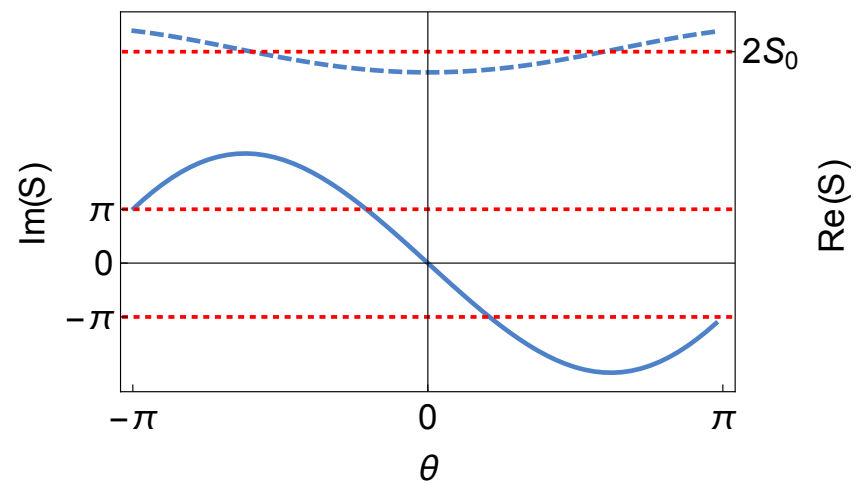

Figure 16. Real and imaginary part of the action of the analytically continued real bounce solution for $p=1$. Under $\theta \rightarrow \theta+2 \pi$, a complex solution goes to the conjugate, reflecting monodromy 2 nature of the solution.

lower separatrix (critical orbit) associated with energy $E_{3}$ in Fig. 10. As $\theta$ is turned on, it interpolates into a smooth saddle of the analytically continued path integral. At $\theta=\pi^{-}$, the real bounce turns into a complex bion. The real part of the action is continuous at $\theta=\pi$, while the imaginary part is discontinuous. Note that the real part of the action of the complex bion is larger than twice the instanton action, $2 S_{I}$. The reason for this is that the bion is associated with the upper separatrix associated with energy $E_{1}$ in Fig. 10 . As for the imaginary part, for $p \notin \mathbb{Z}^{+}$, the ambiguity of the action is related to resurgence, and ambiguity cancellation, and for $p \in \mathbb{Z}^{+}$it is related 


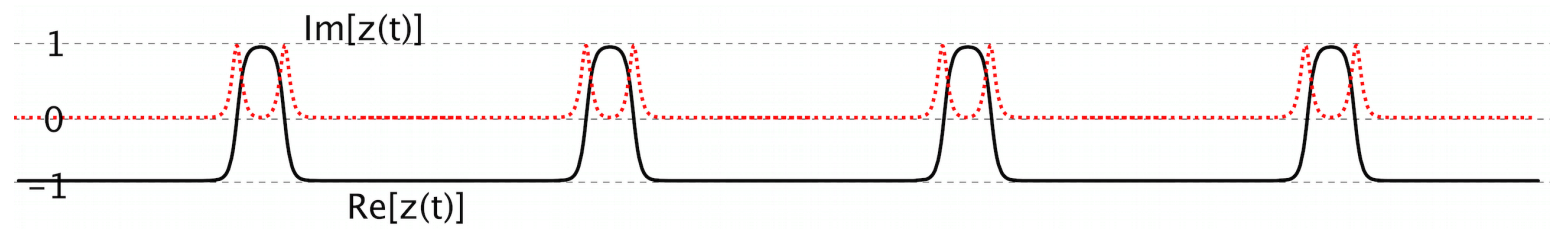

Figure 17. The ground state properties of the theory with fixed number of fermions $\left(N_{f} \geq 1\right)$ is controlled by a dilute gas of complex exact bion saddles, with density $e^{-2 S_{0}}$. Black solid (red dashed) line is the real (imaginary) part of the complex solution. The imaginary part is related to the HTA phenomenon.

to the hidden topological angle.

In fact, as $p$ changes its phase by $2 \pi$ in (5.41), the potential $V_{+}(\mathfrak{x})$ turns back to itself, but two complex bion solutions are interchanged. On the pair of solutions, $\left(z_{\mathrm{cb}}, z_{\mathrm{cb}}^{*}\right)^{T}$, the monodromy matrix acts as

$$
M(2 \pi)=\left(\begin{array}{ll}
0 & 1 \\
1 & 0
\end{array}\right), \quad M(4 \pi)=M^{2}=\left(\begin{array}{ll}
1 & 0 \\
0 & 1
\end{array}\right) .
$$

Thus, the bounce or bion solutions have monodromy of order 2, reflecting the two-fold ambiguity in the choice of the exact solutions for the physical theory. We show in Section 7 that this two-fold ambiguity is related to the two-fold ambiguity in Borel resummation of perturbation theory.

\subsection{Vacuum as a complex bion gas}

Reminder of the bosonic case, ground state wave function vs. instanton gas: It is well-known that in the bosonic (symmetric) double-well potential, the dilute gas of instantons dictates the ground state properties of the system. In particular, the non-perturbative level splitting between the first excited state and the ground state can be shown to be due to instantons. More specifically, the ground state wave function is symmetric with respect to parity, (3.17), and the probability to find the particle on the left and right well is equal. The dilute instanton gas reflects this property. In fact, in the dilute instanton gas, the classical Euclidean particle spends half of its (Euclidean) time at $+a$ and the other half at $-a$, reflecting the symmetry of the ground state wave function.

Ground state wave function vs. bion gas: We have shown that complex bions are exact saddle points of the path integral in theories with fermions $\left(N_{f} \geq 1\right)$. The Euclidean description of one of the vacua of these theories can be viewed as a dilute gas of neutral bions, see Figure 17. The Euclidean configurations reflects the fact that the ground state wave function is not symmetric. Fermion number is conserved, and 


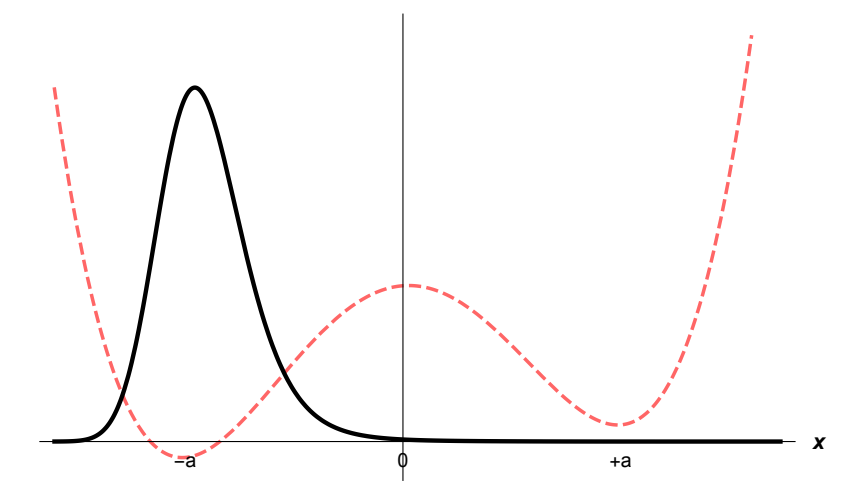

Figure 18. Quantum modified potential (red dashed) for $N_{f} \geq 1$ theories, and one of the two ground state wave function. The probability to find the particle on an $+a$ well is suppressed exponentially, by $e^{-2 S_{I}}$, consistent with the dilute complex bion gas description. As is wellknown, in the bosonic case, $N_{f}=0$, the wave function is equally dominant both on the even and odd sites. Note the absence of any bump in the wave function at $x=+a$.

in any of the two Fock space sectors the particle spends most of its time in the deeper well. More specifically, we showed in (5.43) that one of two ground states is described by

$$
\left|\Psi_{0}\right\rangle|\Omega\rangle=\left|\Psi_{0}\right\rangle \otimes|\downarrow \ldots \downarrow\rangle=a_{1} \underbrace{|R, 0\rangle|\Omega\rangle}_{\text {Ground state in } \mathcal{H}_{\mathrm{R}}}+a_{2} \underbrace{|L, 0\rangle|\Omega\rangle}_{\mathrm{N}_{\mathrm{f}}^{\text {th }} \text {-excited state in } \mathcal{H}_{\mathrm{L}}}+\ldots
$$

and the other ground state is given by $L \leftrightarrow R$. Since the density of the complex bions is $e^{-2 S_{I}}$, the probability to find the particle on the shallow well compared to an deep well is exponentially small. This can also be shown to be the case by using WKB approximation.

$$
\frac{\operatorname{Pr} .(+a)}{\operatorname{Pr} .(-a)} \sim e^{-2 \Delta W / g}=e^{-S_{b}} \sim e^{-2 S_{0}} \quad \text { for one of the ground states. }
$$

For the other ground state, odd and even sites are interchanged. Also, note that the complex bions increase the ground state energy for odd-integer $N_{f}$, (unlike instantons in bosonic theories), and lower it for even-integer $N_{f}$.

What would happen if the complex bion saddle did not contribute? Consider the $N_{f}=1$ theory, corresponding to $\mathcal{N}=1$ supersymmetric QM. In this theory supersymmetry is spontaneously broken, and the ground state energy is given by $E_{\text {gr }} \propto$ $e^{-2 S_{I}}$. This is an effect due to complex bion configuration. If this configuration is not included, one would erroneously conclude that the ground state energy remains zero, and supersymmetry is unbroken. In the original description involving fermions 
and instantons, the ground stage energy is an instanton-anti-instanton $[\mathcal{I} \overline{\mathcal{I}}]$ effect. A rigorous attempt to compute the $[\mathcal{I} \overline{\mathcal{I}}]$ contribution amounts to the complexification of the quasi-zero mode direction, and essentially results in a systematic approximation to the complex bion configuration as described in Section.4.

Complexity of the solution and the hidden topological angle (HTA): Again we consider the case $N_{f}=1$. The ground state energy is given by

$$
E_{\mathrm{gr}} \propto-\left.e^{-2 S_{I} \pm i N_{f} \pi}\right|_{N_{f}=1}=e^{-2 S_{I}}>0 .
$$

Although the action is multi-valued and ambiguous for general non-integer $N_{f}$, it becomes unambiguous for even $N_{f}$. For odd-integer $N_{f}$, it is again unambiguous, because the imaginary part of the action is defined only modulo $2 \pi$, and hence, $\pm \pi$ are actually identified. ${ }^{15}$ However, the fact that $|\operatorname{Im}(S)|=\pi$ is extremely important, because it determines the sign of the ground state energy. Without the HTA, or if the solution were real, we would conclude that the ground state energy must be negative. Indeed, in some of the older literature on instantons it is asserted that non-perturbative saddles always reduce the ground state energy. But this would be inconsistent with the supersymmetry algebra, which implies positive-definiteness of the ground state energy

$$
\left.\left\langle\mathrm{G}_{i}|H| \mathrm{G}_{i}\right\rangle=|Q| \mathrm{G}_{i}\right\rangle\left.\right|^{2} \geq 0
$$

The role of the hidden topological angle (and complexity of the solution) is to restore consistency between supersymmetry algebra and the semi-classical approximation.

\section{Graded formulation: Periodic Sine-Gordon potential}

\footnotetext{
Outline: In this section we construct new exact saddle point solutions for the system whose bosonic potential is the periodic Sine-Gordon potential. In addition to an exact real bounce solution, there is an exact real bion solution, and also an exact complex bion solution. The main new phenomenon is that there is a multi-valued bion solution which is also singular, but has finite action. We show that the singular bion contributes to the path integral, and must be included in the semi-classical analysis in order to obtain results consistent with the Witten index, and the SUSY algebra in the $N_{f} \rightarrow 1$ limit. This resolves an old and controversial issue regarding the inclusion multi-valued, singular saddles in the evaluation of path integral.
}

${ }^{15}$ This aspect of the hidden topological angle is the same as the usual topological $\Theta$-angle in YangMills and other theories. Yang-Mills theory is invariant under CP-at $\Theta=0$ (obvious) and at $\pi$, because $\pm \pi$ are identified. 


\subsection{Quantum modified potential and exact non-BPS solutions}

The Euclidean Lagrangian for the periodic auxiliary potential in the presence of $N_{f}$ Grassmann-valued fermion fields is given by a set of graded Lagrangians, as described in $\S .2 .3$, with $W^{\prime}(z)=-2 a^{2} \sin (z / 2 a)$. The graded Euclidean Lagrangian and energy conservation relations for the periodic system are given by

$$
\begin{aligned}
& \mathcal{L}=\frac{1}{2} \dot{z}^{2}+V(z)=\frac{1}{2} \dot{z}^{2}+2 a^{4}-2 a^{4} \cos ^{2}(z / 2 a)-\frac{p g}{2} a \cos (z / 2 a) \\
& E=\frac{1}{2} \dot{z}^{2}-V(z)=\frac{1}{2} \dot{z}^{2}-2 a^{4}+2 a^{4} \cos ^{2}(z / 2 a)+\frac{p g}{2} a \cos (z / 2 a)
\end{aligned}
$$

for the fermion-number sector $k$, where $p=N_{f}-2 k$, for $k=0, \ldots, N_{f}$. As in the double-well case, the fermion-induced potential is of order $g$.

The inverted potential is shown in Figure 4, along with the physically important saddles. The bounce solution is straightforward, but is not relevant for the ground state properties. The real bion is expected, as it exists as a solution of the ordinary (real) Newton equation in the inverted potential. The surprise is the complex bion solution to the holomorphic Newton equation, which has no counterpart in (real) classical mechanics.

To describe the non-perturbative saddles in the problem, we study the classical solutions in the inverted potential, $-V(z)$, in Euclidean time. The solutions can be found by quadratures:

$$
\int_{t_{0}=0}^{t} d t=-\int_{z_{T}}^{z} \frac{d z}{\sqrt{2(E+V(z))}}=-\int \frac{d z}{\sqrt{2 E+4 a^{4}-4 a^{4} \cos ^{2}(z / 2 a)-p g a \cos (z / 2 a)}} .
$$

Substituting

$$
Z=a \cos (z / 2 a)
$$

the integration takes a form familiar from the Weierstrass elliptic functions:

$$
t-t_{c}=\int_{\cos \left(z_{T} / 2\right)}^{\cos (z / 2)} \frac{d Z}{\sqrt{Z^{4}+\frac{1}{4 a^{2}} p g Z^{3}-\frac{1}{2 a^{2}}\left(E+4 a^{4}\right) Z^{2}-\frac{1}{4} p g Z+a^{4}+\frac{1}{2} E}},
$$

where $t_{c}$ is the integration constant (which becomes the center position modulus of the solution), and $z_{T}$ is the turning point. The mapping (6.3) maps two physically distinct points, $z$ and $-z$, to the same value of $Z$. The inversion of this mapping is twovalued, and requires some care, which will be easy to understand both on physical and mathematical grounds. There will be another multi-valuedness in the story unrelated 
to this, and tied with the nature of the exact complex bion solution. The integral (6.4) can be associated with a complex algebraic curve:

$$
F(Z)=\mathcal{P}^{2}(Z):=Z^{4}+\frac{1}{4 a^{2}} p g Z^{3}-\frac{1}{2 a^{2}}\left(E+4 a^{4}\right) Z^{2}-\frac{1}{4} p g Z+a^{4}+\frac{1}{2} E .
$$

The general solution $z(t)$ for an energy $E$ that corresponds to a turning point $Z_{T}$ (a zero of the polynomial $F(Z)$ ), is given by

$$
z(t)= \pm 2 a \arccos \left(Z_{T}+\frac{\frac{1}{4} F^{\prime}\left(Z_{T}\right)}{\wp\left(t ; g_{2}(E), g_{3}(E)\right)-\frac{1}{24} F^{\prime \prime}\left(Z_{T}\right)}\right)
$$

where $g_{2}(E), g_{3}(E)$ are the associated with algebraic lattice invariants, given by

$$
\begin{aligned}
& g_{2}(E)=\frac{4 a^{4}}{3}+\frac{2}{3} E+\frac{1}{48 a^{4}} E^{2}+\frac{1}{64 a^{2}} g^{2} p^{2}, \\
& g_{3}(E)=-\frac{8 a^{6}}{27}-\frac{2 a^{2}}{9} E-\frac{5}{144 a^{2}} E^{2}+\frac{1}{1728 a^{6}} E^{3}-\frac{1}{192 a^{2}} g^{2} p^{2}-\frac{1}{768 a^{4}} g^{2} p^{2} E .
\end{aligned}
$$

Below, we construct the most interesting solutions: First, a simple bounce solution, and then the complex and real bion solutions which determine the ground state properties of the quantum system.

\subsection{Exact (real) bounce solution}

The "bounce" solution $[5,72,73]$ is a classical solution with Euclidean energy

$$
E=-\frac{1}{2} p g a
$$

equal to the value of the inverted potential at $z / a=2 \pi+4 \pi k$, for $k \in \mathbb{Z}$, the lower of the (real) maxima of the inverted potential. At this energy there are real turning points at $z=z_{T}$ where

$$
\cos \frac{z_{T}}{2 a}=1-\frac{g p}{4 a^{3}}, \quad z_{T}= \pm 2 a \arccos \left(1-\frac{g p}{4 a^{3}}\right)+4 a \pi k, \quad k \in \mathbb{Z}
$$

The bounce solution bounces off the (real) turning point $z_{T}$ at a finite time $t=0$, tending as $t \rightarrow \pm \infty$ to $z / a=2 \pi+4 \pi k$, for $k \in \mathbb{Z}$, see Figure 19 .

With these boundary conditions, the general solution (6.6) simplifies significantly, as the Weierstrass function reduces to a singly-periodic function. We find

$$
\begin{aligned}
z_{\mathrm{bn}}(t) & =2 a \arccos \left(\frac{1-\frac{p g}{8 a^{3}-p g} \cosh ^{2}\left(\omega_{\mathrm{bn}} t\right)}{1+\frac{p g}{8 a^{3}-p g} \cosh ^{2}\left(\omega_{\mathrm{bn}} t\right)}\right) \\
& =4 a \arctan \left(\sqrt{\frac{p g}{8 a^{3}-p g}} \cosh \left(\omega_{\mathrm{bn}} t\right)\right),
\end{aligned}
$$


where $\omega_{\mathrm{bn}}$ is the curvature of the potential at $z=2 \pi a$, given by ${ }^{16}$

$$
\omega_{\mathrm{bn}}=\sqrt{V^{\prime \prime}(2 a \pi)}=a \sqrt{1-\frac{p g}{8 a^{3}}}
$$

Alternatively, we can obtain this solution more directly by substituting into the energy condition $(6.1 \mathrm{~b})$ the ansatz

$$
z(t)=4 a \arctan (f(t))
$$

which produces the following equation for $f(t)$ :

$$
a^{2} \dot{f}^{2}=\frac{1}{8}\left(E+\frac{p g}{2} a\right) f^{4}+\frac{E+4 a^{4}}{4} f^{2}+\frac{1}{8}\left(E-\frac{p g}{2} a\right) .
$$

At the bounce energy, $E=-\frac{p g}{2} a$, the quartic term in $f$ vanishes, and we obtain the solution in (6.10b). The bounce solution clearly satisfies the desired boundary conditions $(\bmod 4 a \pi)$ :

$$
z_{\mathrm{bn}}( \pm \infty)=2 a \pi, \quad z_{\mathrm{bn}}(0)=2 a \arccos \left(1-\frac{g p}{4 a^{3}}\right)
$$

It is physically instructive to rewrite the bounce solution as

$$
z_{\mathrm{bn}}(t)=4 \pi a-4 a\left[\arctan \left(\exp \left[-\omega_{\mathrm{bn}}\left(t-t_{0}\right)\right]\right)+\arctan \left(\exp \left[\omega_{\mathrm{bn}}\left(t+t_{0}\right)\right]\right)\right]
$$

where we have defined

$$
t_{0} \equiv \omega_{\mathrm{bn}}^{-1} \ln \left[\sqrt{\frac{8 a^{3}}{p g}}\left(1+\omega_{\mathrm{bn}} / a\right)\right] \approx \frac{1}{2 a} \ln \left(\frac{32 a^{3}}{p g}\right) .
$$

Thus, the real bounce has the form of a Sine-Gordon instanton at $t=-t_{0}$, followed by a anti-instanton at $t=+t_{0}$, as shown in Figure 19. However, unlike the superposition of the instanton-anti-instanton, which is an approximation, the real bounce solution is exact.

\footnotetext{
${ }^{16}$ The linear frequency on the well at $z=2 \pi a$ (the value of the frequency as one take $E \rightarrow \frac{1}{2} p g a$ limit in the original potential) is equal to the non-linear frequency on the well at $z=0$ for the energy level $E=\frac{1}{2} p g a$.

$$
\omega_{\mathrm{bn}}=\omega_{\text {linear }}^{2 \pi \mathrm{a}}\left(E=\frac{1}{2} p g a\right)=\omega_{\text {non-linear }}^{0}\left(E=\frac{1}{2} p g a\right)
$$

This identity is a consequence of the relation (5.12) for the periods of the complex algebraic curve. Physically, in the singly periodic limit of the Weierstrass function, $i \omega_{\text {non-linear }}^{0}\left(E=\frac{1}{2} p g a\right.$ ) is the (pure imaginary) frequency which remains finite, while the other purely real frequency tends to zero.
} 


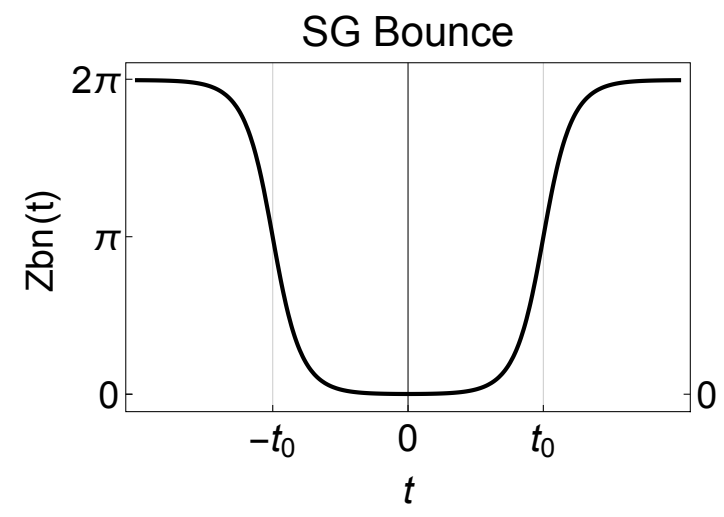

Figure 19. Bounce solution for the Sine-Gordon case, for $a=1$. The size of separation (or the size of plateau is $2 t_{0} \approx a^{-1} \ln \left(\frac{32 a^{3}}{p g}\right)$.

As in the double-well case, the bounce is parametrically flat. The bounce spends a parametrically large time in the vicinity of the turning point (unlike instantons). The size of the flat-bounce is given by

$$
2 t_{0} \approx m_{b}^{-1} \ln \left(\frac{32 a^{3}}{p g}\right)
$$

where $m_{b}=a$ in the SG case. Note that the size of the bounce obtained in the exact solution agrees precisely with the integration over the quasi-zero mode thimble $\Gamma^{\mathrm{qzm}}$. In particular, the scale (6.18) provides the dominant support of the QZM integral (4.3), and corresponds to the saddle of the QZM-integration.

Finally, the action of the bounce solution is given by

$$
\begin{aligned}
S_{\mathrm{bn}} & =\frac{16 a^{3}}{g} \sqrt{1-\frac{g p}{8 a^{3}}}-2 p \operatorname{arctanh}\left[\sqrt{1-\frac{g p}{8 a^{3}}}\right] \\
& =\frac{16 a^{3}}{g} \sqrt{1-\frac{g p}{8 a^{3}}}-p \log \left[\frac{1+\sqrt{1-\frac{g p}{8 a^{3}}}}{1-\sqrt{1-\frac{g p}{8 a^{3}}}}\right] \\
& =\frac{16 a^{3}}{g}-p \ln \left(\frac{32 a^{3}}{p g}\right)+\mathcal{O}(g) .
\end{aligned}
$$

Similar to DW, the action of the real bounce is less than two-times instanton action, $2 S_{I}$. The reason for this is that the bounce is associated with the lower separatrix associated with energy (6.8).

\subsection{Exact real bion solution}

The real bion solution has Euclidean energy

$$
E=\frac{1}{2} p g a,
$$




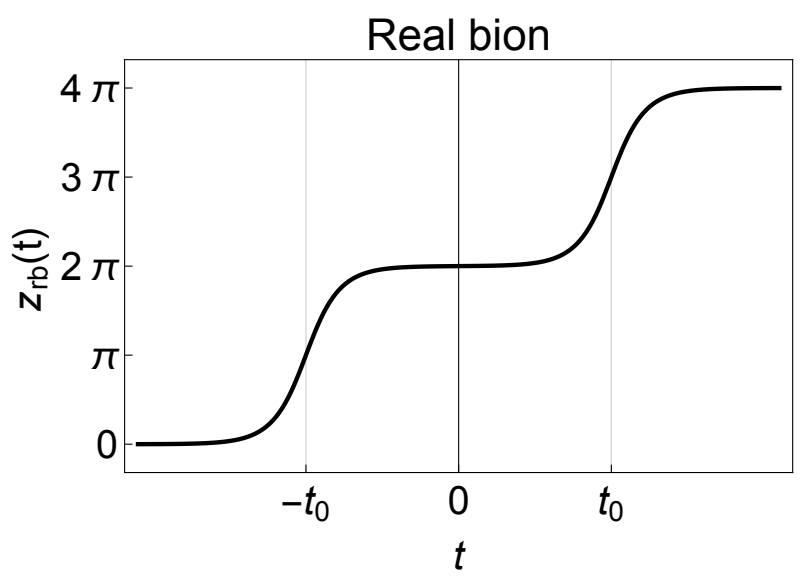

Figure 20. Exact real bion solution, interpolating from 0 to $4 \pi$, for $a=1$. The plateau in the middle is associated with the characteristic size of the solution $2 t_{0} \approx a^{-1} \ln \left(\frac{32 a^{3}}{p g}\right)$. The ground state properties of the quantum theory in leading order semi-classics are described in terms of a dilute gas of real bions and complex bions.

equal to the inverted potential at its global maxima: $z / a=4 \pi k$, for $k \in \mathbb{Z}$. Qualitatively, the classical particle, after spending an infinite amount of time at the global maximum, rolls down at some instant in time (just like the instanton), and then spends a parametrically large but finite amount of time in the vicinity of the local maximum near $2 \pi a$, and continue its path, reaching to the next equivalent global maximum at $4 \pi a$ after infinite time. The exact real bion solution is shown in Fig. 20.

At the energy level of upper separatrix (6.20), the general solution (6.6) simplifies significantly, as the Weierstrass function reduces to a singly-periodic function, leading to

$$
z_{\mathrm{rb}}(t)=2 \pi a+4 a \arctan \left(\sqrt{\frac{p g}{8 a^{3}+p g}} \sinh \left(\omega_{\mathrm{rb}} t\right)\right)
$$

where $\omega_{\mathrm{bn}}$ is the curvature of the potential at $z=0$ and is given by

$$
\omega_{\mathrm{rb}}=\sqrt{V^{\prime \prime}(0)}=a \sqrt{1+\frac{p g}{8 a^{3}}} .
$$

This satisfies the boundary conditions:

$$
z_{\mathrm{rb}}(-\infty)=0 \quad, \quad z_{\mathrm{rb}}(0)=2 \pi a \quad, \quad z_{\mathrm{rb}}(+\infty)=4 \pi a
$$

Alternatively, applying the transformation $z(t)=2 \pi a+4 a \arctan f(t)$ to $(6.1 \mathrm{~b})$, we obtain

$$
a^{2} \dot{f}^{2}=\frac{1}{8}\left(E-\frac{p g}{2} a\right) f^{4}+\frac{E+4 a^{4}}{4} f^{2}+\frac{1}{8}\left(E+\frac{p g}{2} a\right) .
$$


At $E=\frac{p g}{2} a$ the quartic term in $f$ vanishes, and we obtain the solution in (6.25). It is instructive to rewrite the real bion solution as

$$
z_{\mathrm{rb}}(t)=2 a \pi+4 a\left(\arctan \left(\exp \left[\omega_{\mathrm{rb}}\left(t+t_{0}\right)\right]\right)-\arctan \left(\exp \left[-\omega_{\mathrm{rb}}\left(t-t_{0}\right)\right]\right)\right)
$$

where $t_{0}$ is

$$
t_{0}=\omega_{\mathrm{rb}}^{-1} \ln \left[\sqrt{\frac{8 a^{3}}{p g}}\left(1+\omega_{\mathrm{rb}} / a\right)\right] \approx \frac{1}{2 a} \ln \left(\frac{32 a^{3}}{p g}\right)
$$

Hence, one observes that the real bion solution is the exact version of the correlated instanton-instanton event described in Section 4. Despite the fact that the $[\mathcal{I I}]$ event is an approximate solution in the original formulation, the real bion solution is exact. In the regime $p g \ll a^{3}$, the relation between the two is:

$$
z_{\mathrm{rb}}(t) \approx x_{\mathcal{I}}\left(t+t_{0}\right)+x_{\mathcal{I}}\left(t-t_{0}\right)+2 a \pi, \quad \text { for } p g \ll a^{3} .
$$

The characteristic size of the real bion solution (6.26) agrees with (4.3), which is the saddle point of the QZM-thimble $\Gamma_{+}^{\text {qzm }}$ integration in the original formulation, see Figure 9.

The action of the real bion solution is given by

$$
\begin{aligned}
S_{\mathrm{rb}} & =\frac{16 a^{3}}{g} \sqrt{1+\frac{g p}{8 a^{3}}}+2 p \operatorname{arctanh}\left[1 / \sqrt{1+\frac{g p}{8 a^{3}}}\right] \\
& =\frac{16 a^{3}}{g}+p \ln \left(\frac{32 a^{3}}{p g}\right)+\mathcal{O}(g) .
\end{aligned}
$$

The action of the real bion is larger than twice the instanton action, $2 S_{I}$. The reason for this is that the real bion is associated with the upper separatrix with energy (6.20), and the area underneath the separatrix in classical phase space is larger than the instanton-anti-instanton separatrix.

\subsection{Exact complex bion solution}

To find the exact complex bion solution, we impose the boundary condition that at $t=0$ the solution starts at one of the complex turning points

$$
z_{T}=2 a \pi \pm 2 i a \operatorname{arccosh}\left(1+\frac{p g}{4 a^{3}}\right)
$$

for which the Euclidean energy is

$$
E=\frac{1}{2} p g a
$$




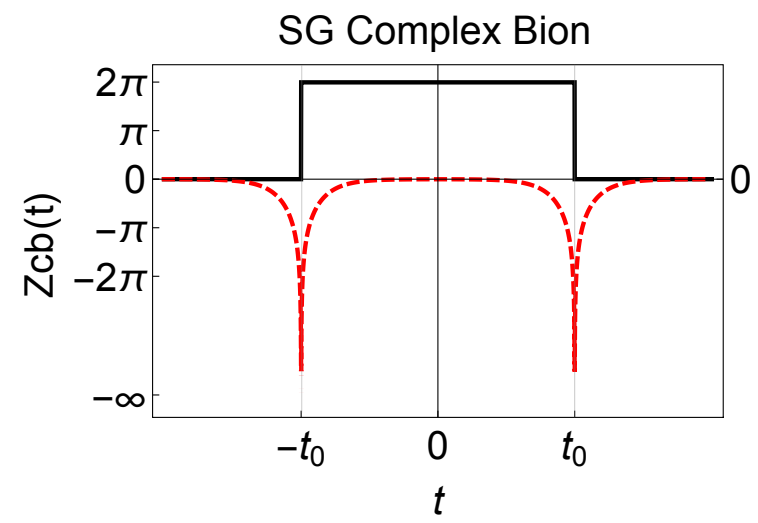

Figure 21. Real (black) and imaginary (red) parts of the complex bion for the Sine-Gordon case, The separation is $2 t_{0} \approx a^{-1} \ln \left(\frac{32 a^{3}}{p g}\right)$. The singular solution smooths out upon analytic continuation $p \rightarrow p e^{i \theta}$.

Then, from the general solution (6.6), or by substituting an appropriate ansatz, we find the two complex conjugate complex bion solutions to be

$$
z_{\mathrm{cb}}=2 a \pi \pm 4 a \arctan \left(i \sqrt{\frac{p g}{8 a^{3}+p g}} \cosh \left(\omega_{\mathrm{cb}} t\right)\right)
$$

where $\omega_{\mathrm{cb}}$ is the frequency of the system at $z=0$ well, given by:

$$
\omega_{\mathrm{cb}}=\sqrt{V^{\prime \prime}(0)}=a \sqrt{1+\frac{p g}{8 a^{3}}} .
$$

This expression is very similar to that of the real bion, which is related to the fact that both solutions start at the global maximum. The solution is complex, and its real and imaginary parts are plotted in Figure 21.

Note that the real part jumps, and the imaginary part is correspondingly singular, at a particular $\pm t_{0}$, where $2 t_{0}$ can be interpreted in terms of the size of this configuration. In the past, such configurations would not even be seriously considered as legitimate saddles due to "disturbing" discontinuity and singularity. But the story is more interesting and beautiful, and such saddles do contribute to the path integral. The physics of this jump behavior is discussed below in Section 6.5. It is easy to show that the complex solution in SG can also be written exactly in terms of an instantonanti-instanton pair as

$$
z_{\mathrm{cb}}(t)=2 \pi a \pm 4 a\left[\arctan \left(\exp \left(\omega_{\mathrm{cb}}\left(t+t_{0}\right)\right)\right)+\arctan \left(\exp \left(-\omega_{\mathrm{cb}}\left(t-t_{0}\right)\right)\right)\right],
$$

where

$$
t_{0}=\omega_{\mathrm{cb}}^{-1} \ln \left[\sqrt{-\frac{8 a^{3}}{p g}}\left(1+\omega_{\mathrm{bn}} / a\right)\right] \approx \frac{1}{2 a}\left[\ln \left(\frac{32 a^{3}}{p g}\right) \pm i \pi\right]
$$


The complex bion solution is the exact form of the $[\mathcal{I} \overline{\mathcal{I}}]_{ \pm}$correlated 2-event found by integrating over the quasi-zero mode thimble, $\Gamma_{-}^{\mathrm{qzm}}$. Again, rather remarkably, the approximation over the complexified quasi-zero mode thimble (4.7) has all the correct features of the complex bion solution, i.e., it is a systematic approximation to the exact solution. In particular, the complex quasi-zero mode "separation" saddle of the integration is the same as the size of the plateau, $\tau^{*} \approx 2 t_{0}$, which is an exact feature of the exact solution.

The action of the complex bion is

$$
\begin{aligned}
S_{\mathrm{cb}} & =\frac{16 a^{3}}{g} \sqrt{1+\frac{g p}{8 a^{3}}}+2 p \operatorname{arctanh}\left[\sqrt{1+\frac{g p}{8 a^{3}}}\right] \\
& =\frac{16 a^{3}}{g}+p \ln \left(\frac{32 a^{3}}{p g}\right) \pm i p \pi+\mathcal{O}(g) .
\end{aligned}
$$

The real part of the action of the complex bion is equal to the action of the real bion, as can be seen by inspecting (6.35a) and (6.28b). Furthermore, it is larger than two-times instanton action, $2 S_{I}$ since it is associated with upper separatrix. They differ in the imaginary part of the action of the complex bion. If $p=1,2, \ldots$, then, the complex action is unambiguous (because the imaginary part of the bion action is well-defined modulo $2 \pi$ ), and relates to the hidden topological angle [26]. If $p$ is non-integer, then, the action is multi-fold ambiguous, similar to the action of the DW complex bion in (5.38) which cancels with the ambiguity associated with the non-Borel resummability of the perturbation theory, as described in more detail in Section 7.

Equality of the real bion and real part of the complex bion actions: The equality of the real bion action $S_{\mathrm{rb}}$ with the real part of the complex bion $S_{\mathrm{cb}}$ action can also be shown in a more elegant manner, (without doing computation) by using contour deformation. The complex bion increases the ground state energy by an amount $\sim e^{-\operatorname{Re}\left(S_{\mathrm{cb}}\right)}$, and the real bion lowers it by $\sim e^{-S_{\mathrm{rb}}}$. The ground state energy will remain zero (as required by SUSY) if and only if $S_{\mathrm{rb}}=\operatorname{Re}\left(S_{\mathrm{cb}}\right)$.

The actions of the real and complex bion are computed along the contours $\mathcal{C}_{1}$ and $\mathcal{C}_{2}$ defined in Figure 22:

$$
\begin{aligned}
& S_{\mathrm{rb}}=\int_{0}^{4 \pi} d z \sqrt{2 V(z))}=\int_{\mathcal{C}_{1}} \sqrt{2 V(z)} \\
& S_{\mathrm{cb}}=2 \int_{0}^{z_{T}} d z \sqrt{2 V(z)}=2 \int_{\mathcal{C}_{2}} \sqrt{2 V(z)},
\end{aligned}
$$

where $V(z)$ is the quantum modified potential and $z_{T}$ is the complex turning point. On the other hand, $S_{\mathrm{cb}}^{*}$ can be written as an integral over the contour $\mathcal{C}_{2}^{\prime}: S_{\mathrm{cb}}^{*}=$ $2 \int_{0}^{\bar{z}_{T}} d z \sqrt{2 V(z)}=2 \int_{\mathcal{C}_{2}^{\prime}} \sqrt{2 V(z)}$. Because $V(4 a \pi-z)=V(z)$ we can change variables, 


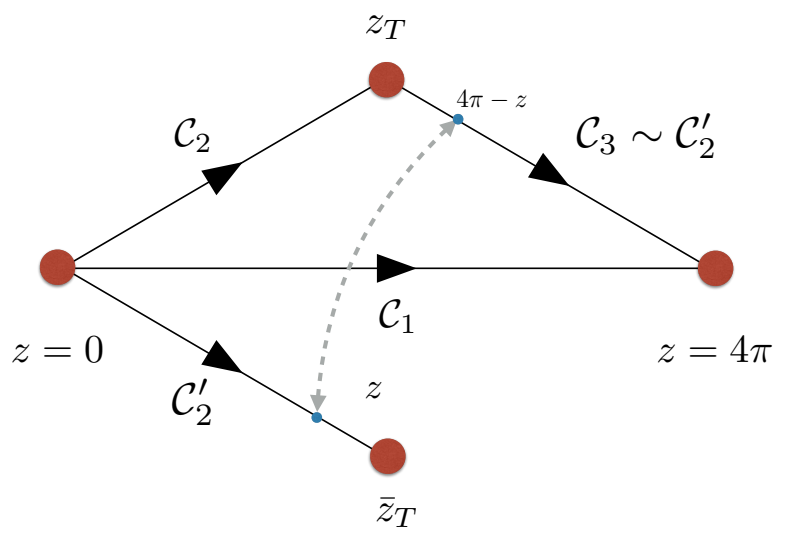

Figure 22. The complex $z$-plane. $\mathcal{C}_{1}$ is associated with real bion, and $\mathcal{C}_{2}$ is associated with the complex bion with complex turning point. Here we have taken $a=1$.

$z \rightarrow 4 a \pi-z$, and write $\int_{\mathcal{C}_{2}^{\prime}}=\int_{\mathcal{C}_{3}}$, where the contour $\mathcal{C}_{3}$ is shown in Figure 22. Therefore

$$
\operatorname{Re} S_{\mathrm{cb}}=\frac{1}{2}\left(S_{\mathrm{cb}}+S_{\mathrm{cb}}^{*}\right)=\int_{\mathcal{C}_{2} \cup \mathcal{C}_{3} \equiv \mathcal{C}_{1}} d z \sqrt{2 V}=S_{\mathrm{rb}} .
$$

This proves the equality of the real parts of the actions for any positive value of $p=N_{f}$. When we analytically continue $p \rightarrow p e^{i \theta}$, this equality breaks down.

\subsection{Analytic continuation: From real bounce to complex bion}

As in the double-well system, the complex bion can be obtained by analytic continuation of the real bounce solution. Let us consider the analytic continuation of the Sine-Gordon theory to the complex $p$ plane, namely:

$$
p \rightarrow p e^{i \theta} \in \mathbb{C}
$$

The interesting aspect is the following. Start with $V_{+}(z)=\frac{1}{2}\left(W^{\prime}(z)\right)^{2}+\frac{p g}{2} W^{\prime \prime}(z)$, and consider $\theta \neq 0$. At $\theta=\pi$, we reach to the theory described by the potential $V_{-}(z)$, which is isospectral to $V_{+}(z)$, because the two systems are related by a translation by $2 \pi a$, see (2.38). Upon analytic continuation all the way to $\theta=\pi$ : (i) The local maximum of the $-V_{+}(z)$ (along real axis) becomes the global maximum of $-V_{-}(z)$. (ii) The global maximum of the $-V_{+}(z)$ becomes local maximum of $-V_{-}(z)$. (iii) The real turning point of the real bounce solution in the $-V_{+}(z)$ system turns into a complex turning point in the $-V_{-}(z)$ system.

The analytic continuation of the bounce solution to arbitrary $\theta$ is clearly still an exact solution of the holomorphic Newton equations (1.20) in the inverted potential. 
$\theta=0$
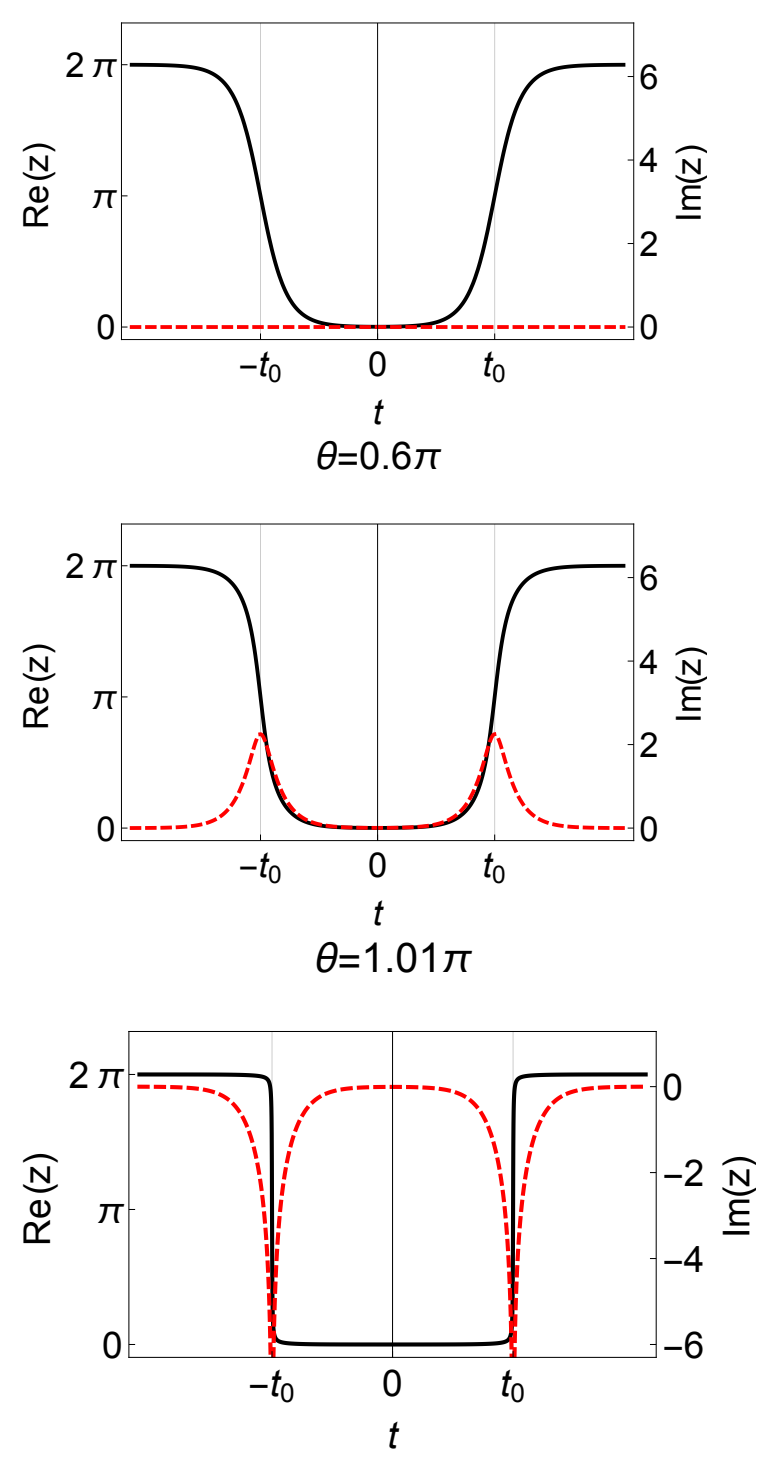
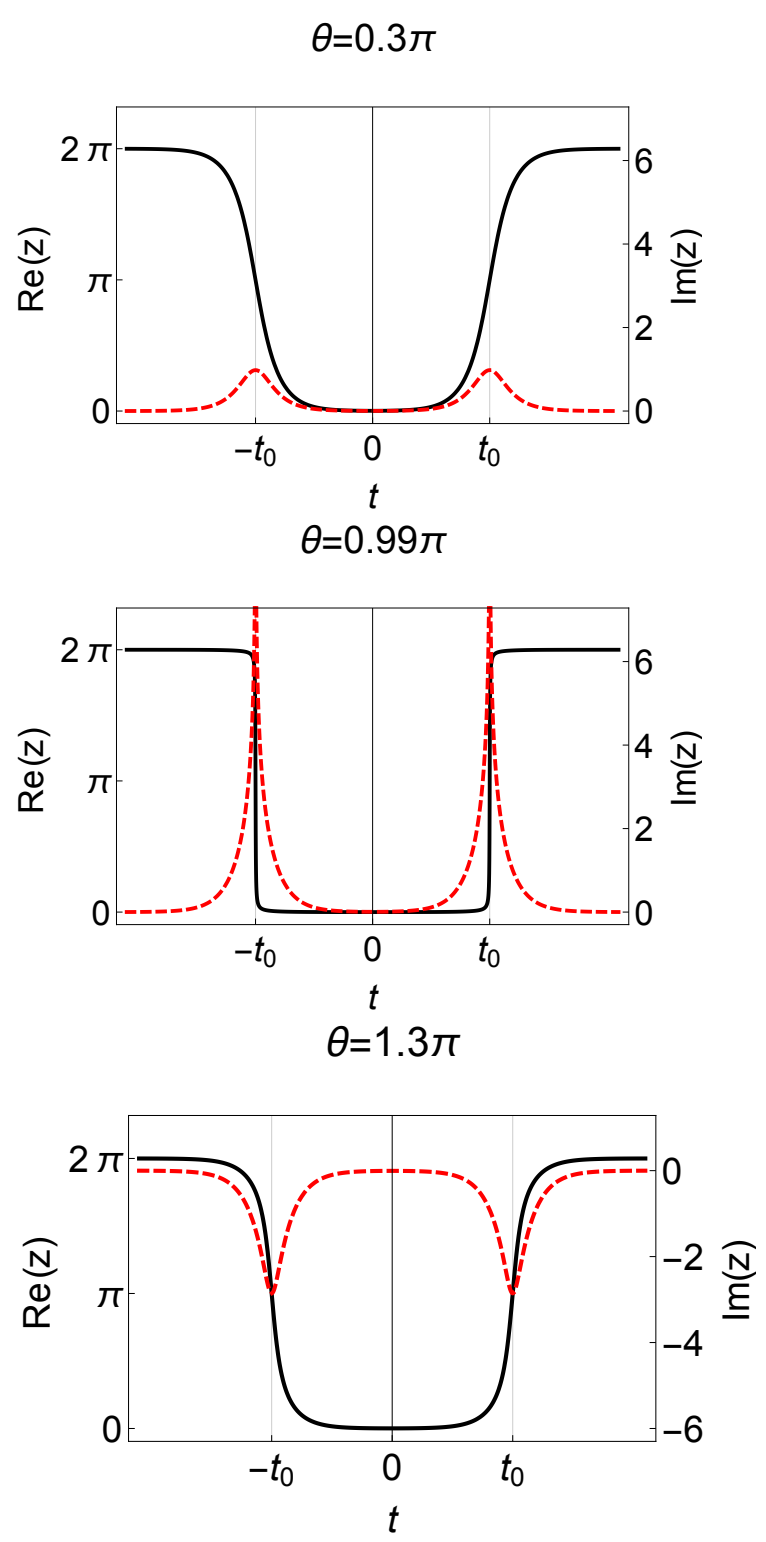

Figure 23. Complex saddle solution $z(t, \theta)$. Black lines show $\operatorname{Re}(z)$ and dashed red lines show $\operatorname{Im}(z)$. We have chosen $p g / a^{3} \ll 1$ and set $a=1$. $\theta=0$ corresponds to the real bounce, and $\theta=\pi^{\mp}$ corresponds to the complex bion. The characteristic size of the solution is always $2 t_{0} \approx \ln \left(\frac{32 a^{3}}{p g}\right)$.

The solutions are obviously complex for general $\theta$, with real and imaginary parts plotted in Figure 23 for various values of $\theta$. The solution obtained via analytic continuation of 


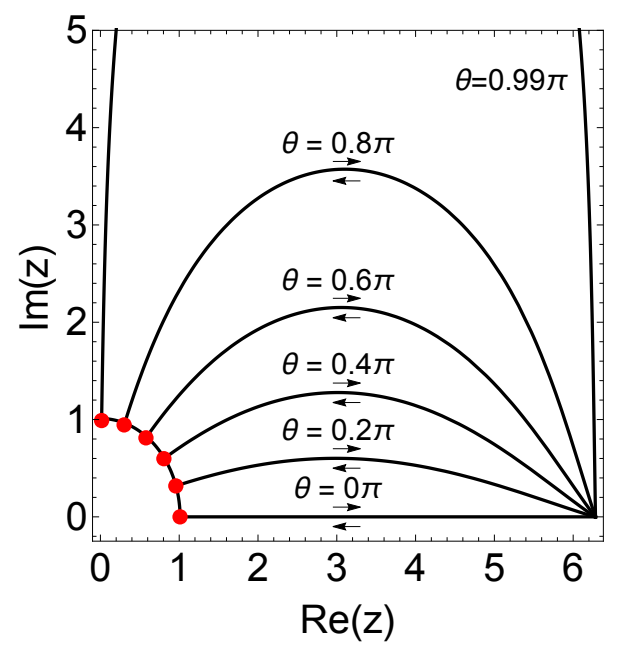

Figure 24. Parametric plot of the real and imaginary part of the analytic continuation of the real bounce solution, $p \rightarrow p e^{i \theta}$. $\theta=0$ correspond to real bounce solution, with a real turning point. As one dials theta, the turning point becomes complex. At $\theta=\pi^{-}$, the real bounce turns into a complex bion, and the turning points becomes complex.

the bounce is:

$$
z(t, \theta)=4 a \arctan \left(\sqrt{\frac{p g e^{i \theta}}{8 a^{3}-p g e^{i \theta}}} \cosh \left(\sqrt{1-\frac{p g e^{i \theta}}{8 a^{3}}} a t\right)\right) .
$$

The solution is smooth for all $\theta \in[0, \pi)$, but exhibits two-valuedness for $\theta=\pi$, see Figure 23, which will be discussed below. It satisfies the correct boundary conditions:

$$
z(t= \pm \infty, \theta)=2 a \pi, \quad z(t=0, \theta)=z_{T}=2 a \arccos \left(1-\frac{g p e^{i \theta}}{4 a^{3}}\right)
$$

where $z_{T}$ is the complex turning point, shown in Figure 24. We can calculate the action which is also multi-valued function at $\theta=\pi$. In the $g p /\left(8 a^{3}\right) \lesssim 1$ regime, the action is is analytic in the cut plane,

$$
S(g, \theta)=\frac{16 a^{3}}{g} \sqrt{1-\frac{g p e^{i \theta}}{8 a^{3}}}-2 p e^{i \theta} \operatorname{arctanh}\left[\sqrt{1-\frac{g p e^{i \theta}}{8 a^{3}}}\right],
$$

and two-valued for $\theta=\pi$. The real and imaginary part of the action is plotted in Figure 25. The real part is continuous, with a cusp at $\theta=\pi$. The imaginary part is also continuous except for a discontinuity at $\theta=\pi$ (interpreted below). Note that on general grounds, the action is well defined only up to $2 \pi$ shifts in its imaginary part, as reflected in Figure 25. 


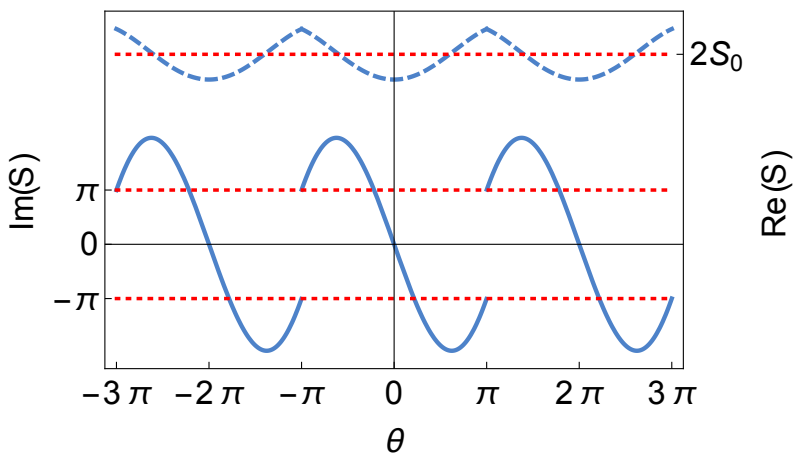

Figure 25. Action of the analytic continuation of the real bounce solution under $p \rightarrow p e^{i \theta}$. At $\theta=\pi^{-}$, the real bounce turns into a complex bion. The real part of the action is continuous at $\theta=\pi$, while the imaginary part is discontinuous. This effect, for $p \neq 1,2, \ldots$ is related to resurgence, and ambiguity cancellation, and for $p=1,2, \ldots$, it is related to the hidden topological angle.

\subsection{Physical Role of Singular, Multi-valued Solutions}

At $\theta=\pi$, the potential is again real, and the complex saddles become a multi-valued complex bion solution. Let us now discuss what happens at $\theta=\pi$ in more detail. We can consider the behavior of the solution at $\theta=\pi \mp \epsilon$ with $\epsilon>0$. The multi-valued complex bion solution is given by

$$
z^{ \pm}(t)=z(t, \theta=\pi \pm \epsilon)
$$

This solution is actually not only multi-valued, it is also singular. (The underlying reason is discussed in the next section.)

In the formalism of real path integration, even the real part of such configurations would not contribute to the path integral as saddle points. Clearly, there are singular configurations in the real path integral (and these are actually generic), but there cannot exist singular saddles with finite action. The simple reason for this is that the kinetic term $\int \frac{1}{2} \dot{x}^{2}$ blows up for a singular or discontinuous configuration. But in complex path integration the situation is different. The kinetic term is now replaced by:

$$
\int \frac{1}{2} \dot{z}^{2}=\int \frac{1}{2}\left(\dot{x}^{2}-\dot{y}^{2}\right)+2 i \int \dot{x} \dot{y} .
$$

Even if the real and imaginary part of a solution $z(t)$ are singular or discontinuous, the action may remain finite provided the real and imaginary parts lead to cancellations in the action. For the holomorphic path integral this is what is taking place at $\theta=\pi$, see Figure 25. 
Remembering that $\operatorname{arctanh}(z / a)$ is analytic in the cut-plane $\mathbb{C} \backslash \mathfrak{c u t}$, and doublevalued along the cut $z / a \in(-\infty,-1) \cup(1, \infty)$ we have

$$
\operatorname{arctanh}(z / a)= \begin{cases}+\frac{1}{2} \ln \left(\frac{z+a}{z-a}\right) \pm \frac{1}{2} \pi i, & z / a \in(-\infty,-1) \cup(1, \infty), \\ +\frac{1}{2} \ln \left(\frac{z+a}{z-a}\right), & z / a \in \mathbb{C} \backslash(-\infty,-1) \cup(1, \infty) .\end{cases}
$$

The action of the complex bion acquires an unambiguous real part and a discontinuous imaginary part at $\theta=\pi$. Both are shown in Figure 25. The action of the complex bion, in its exact form (6.35a), can be rewritten along the cut as

$$
\begin{array}{rlrl}
S_{\mathrm{cb}}^{ \pm}=\frac{S(g)}{g} & =\frac{16 a^{3}}{g} \sqrt{1+\frac{g p}{8 a^{3}}}+2 p \ln \left[\frac{\sqrt{1+\frac{g p}{8 a^{3}}}+1}{\sqrt{1+\frac{g p}{8 a^{3}}}-1}\right] \pm i p \pi \\
& =\operatorname{Re}\left[S_{\mathrm{cb}}\right] \pm i p \pi, & \operatorname{Im}\left[S_{\mathrm{cb}}\right]= \pm p \pi .
\end{array}
$$

Before making a few physical remarks on the implication of the complex saddles in the physical theory, it is useful to state the amplitudes associated with the real bounce, real bion and complex bion in the weak coupling regime:

$$
\begin{aligned}
I_{\mathrm{bn}} & \sim\left(\frac{p g}{32 a^{3}}\right)^{-p} e^{-2 S_{I}}, \\
I_{\mathrm{rb}} & \sim\left(\frac{p g}{32 a^{3}}\right)^{+p} e^{-2 S_{I}}, \\
I_{\mathrm{cb}}^{ \pm} & \sim\left(\frac{p g}{32 a^{3}}\right)^{+p} e^{-2 S_{I} \pm i p \pi},
\end{aligned}
$$

This is in precise agreement with the approximate amplitudes of the two-events obtained in Section 4 upon integration over the appropriate QZM-thimbles, $\Gamma^{\mathrm{qzm}}$, in the weak coupling regime. A few remarks are in order:

- The exact solution (6.40) is discontinuous and singular at $\theta=\pi$, but the real part of the action is continuous. The imaginary part of the action jumps, making the action, as well as the complex bion amplitude $I_{\mathrm{cb}}^{ \pm}$two-fold ambiguous for general values of $p$, namely, $p \in \mathbb{R}-\{1,2, \ldots\}$ : For the consistency of the theory this discontinuity must be canceled by the ambiguity of the (left/right) Borel resummation of perturbation theory. ${ }^{17}$

- For integer values, $p=1,2, \ldots$, where $p=1$ corresponds to supersymmetric QM, the ambiguity in the amplitude of complex bion disappears:

$$
I_{\mathrm{cb}}^{ \pm} \sim e^{-S_{c b}^{r}} e^{ \pm i \pi}= \begin{cases}+e^{-S_{c b}^{r}} & p: 2,4, \ldots, \\ -e^{-S_{c b}^{r}} & p: 1,3, \ldots .\end{cases}
$$

\footnotetext{
${ }^{17}$ Discontinuities of this type in saddle amplitudes have led to some concern in the literature, see footnote 33 of [51]. We discuss this important issue and the resolution of the problem in Section 8.1.
} 
In theories for which $p$ is an odd-integer there is a $\mathbb{Z}_{2}$-worth of hidden topological angle (HTA) discussed in our recent work [26]. For example, for $p=1$, this configuration gives a positive contribution to the ground state energy. But there exists another saddle in the problem, the real bion discussed in Section 6.3, which gives a negative contribution to the ground state energy, such that the combination of the two cancels exactly. In this potential, supersymmetry is actually unbroken and the vanishing of the ground state energy relies on the existence of this complex multi-valued, discontinuous saddle in addition to the real bion saddle.

\subsection{Physics of the complex bion solution, multi-valuedness and singularity}

One may wonder why the complex bion solution exhibits singular behavior in the SineGordon system, in contrast with the complex bion in the double well potential discussed in Section 5.3, particularly given that the solution was found by mapping the problem to the double well potential.

An elementary way to understand the difference between the double well and the Sine-Gordon systems is to consider the problem of integrating the classical equations of motion, starting at some complex turning point $z_{T}$ of the potential $V(z)$ at time $t=0$ :

$$
z(t)=z_{T}+t \dot{z}(0)+\frac{t^{2}}{2} \ddot{z}(0)+\frac{t^{3}}{3 !} \dddot{z}(0)+\ldots
$$

If the initial point, $z(0)=z_{T}$ is a turning point, then $\dot{z}(0)=0$, so the first step of the evolution is governed by

$$
\ddot{z}(0)=\left.\frac{\partial V}{\partial z}\right|_{t=0} .
$$

It is easy to see that for the double-well case, $\ddot{z}(0)=2 z_{T}^{3}-z_{T} a^{2}+p g$ is complex, with both non-vanishing real and imaginary parts. Thus the evolution moves away from the complex turning point in the complex plane, ending at the critical point $z_{1}^{\mathrm{cr}}$. This can be seen pictorially in Figure 26, which shows the complex bion for the double-well "rolling" on the surface of the real part of the inverted potential. ${ }^{18}$

\footnotetext{
${ }^{18}$ Note that, in contrast to naive intuition, the particle does not fall into the infinitely deep well. The force on a classical particle in the inverted potential is not (minus) the gradient, $-\vec{\nabla}\left(-V_{\mathrm{r}}\right)$. Instead, $\ddot{x}=-\partial_{x}\left(-V_{\mathrm{r}}\right), \ddot{y}=+\partial_{y}\left(-V_{\mathrm{r}}\right)$, notice the crucial relative minus sign, as discussed around (1.17). The force in the $y$-direction is negated with respect to the usual equations of motion due to holomorphy. As a result, a particle which would, according to the regular Newton equation in 2 d, "roll down" into a well, may instead, "rolls up". This is the reason that the classical (holomorphic) particle in the inverted potential does not fall into the well in Figure 26.
} 


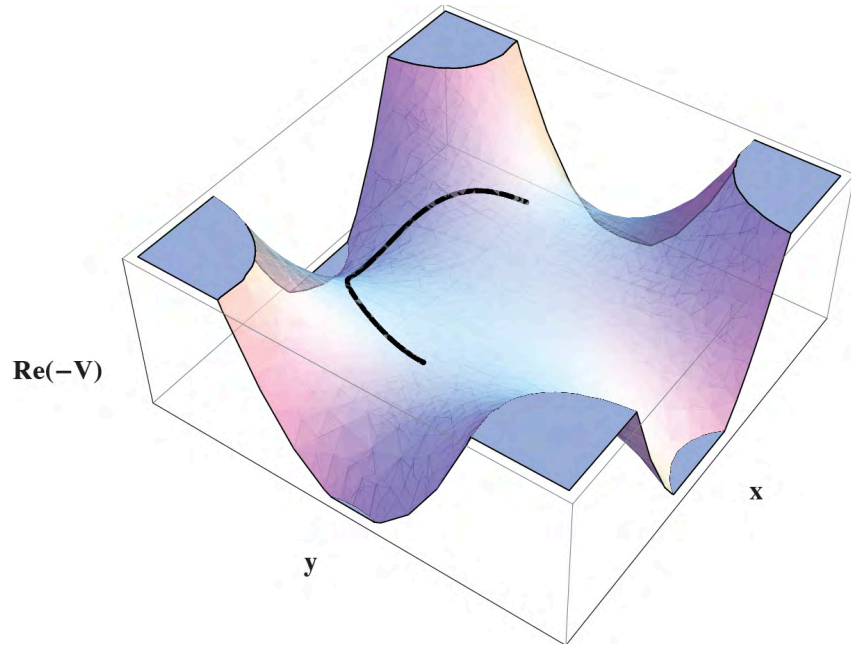

Figure 26. Complex bion rolling on the real part of the inverted potential for the double-well case with $a=1$, and $p g=1 / 10$. The trajectory begins for $t=0$ at the complex turning point and continues to the critical point $z_{1}^{\mathrm{cr}}$ as $t \rightarrow \pm \infty$. The motion is perfectly smooth.

By contrast, for the Sine-Gordon system, starting at the complex turning point $z_{T} / a=2 \pi+2 i \operatorname{arccosh}\left(1+\frac{p g}{4 a^{3}}\right)$, we have

$$
\ddot{z}(0)=a^{2} \sin \left(z_{T} / a\right)+\frac{p}{4} a^{2} \sin \left(z_{T} / 2 a\right)=-2 i a^{2} \sqrt{\frac{p g}{2 a^{3}}}\left(1+\frac{p g}{8 a^{3}}\right)^{3 / 2},
$$

which is pure imaginary. This pattern propagates through the entire series in (6.49) and is a consequence of the symmetry (6.54): all odd derivatives of $z(t)$ vanish at $t=0$, while even derivatives are all pure imaginary. Thus, the real part of $z$ must remain constant; it cannot change from its initial value of $2 a \pi$, except when $\operatorname{Im}(z)$ reaches infinity, as it does in a finite time $t_{0}$, and at this point the real part of $z$ can jump by $2 a \pi$. This can be seen in the "rolling" of the classical particle shown in Figure 27. The particle rolls out along the ridge at $\operatorname{Re}(z)=2 a \pi$, reaching the point at infinity in finite time, and then rolls back along the ridge at $\operatorname{Re}(z)=0$, reaching the critical point at $z=0$ at $t= \pm \infty$. The sudden jump at infinity may be regularized by analytically continuing $p$ in the potential to have a phase, as discussed in the previous subsection. In this case, the jump at infinity is smoothed out.

\section{Symmetry and multi-valuedness (or an alternate story for Buridan's don-} key): There is another more general way to see why the discontinuity and singularity occurs. First, in classical mechanics (and its holomorphic version) uniqueness of solutions is guaranteed for any motion given the initial conditions. This means that the turning point defined by $-V\left(z_{T}\right)=E$ uniquely defines the trajectory of motion in 


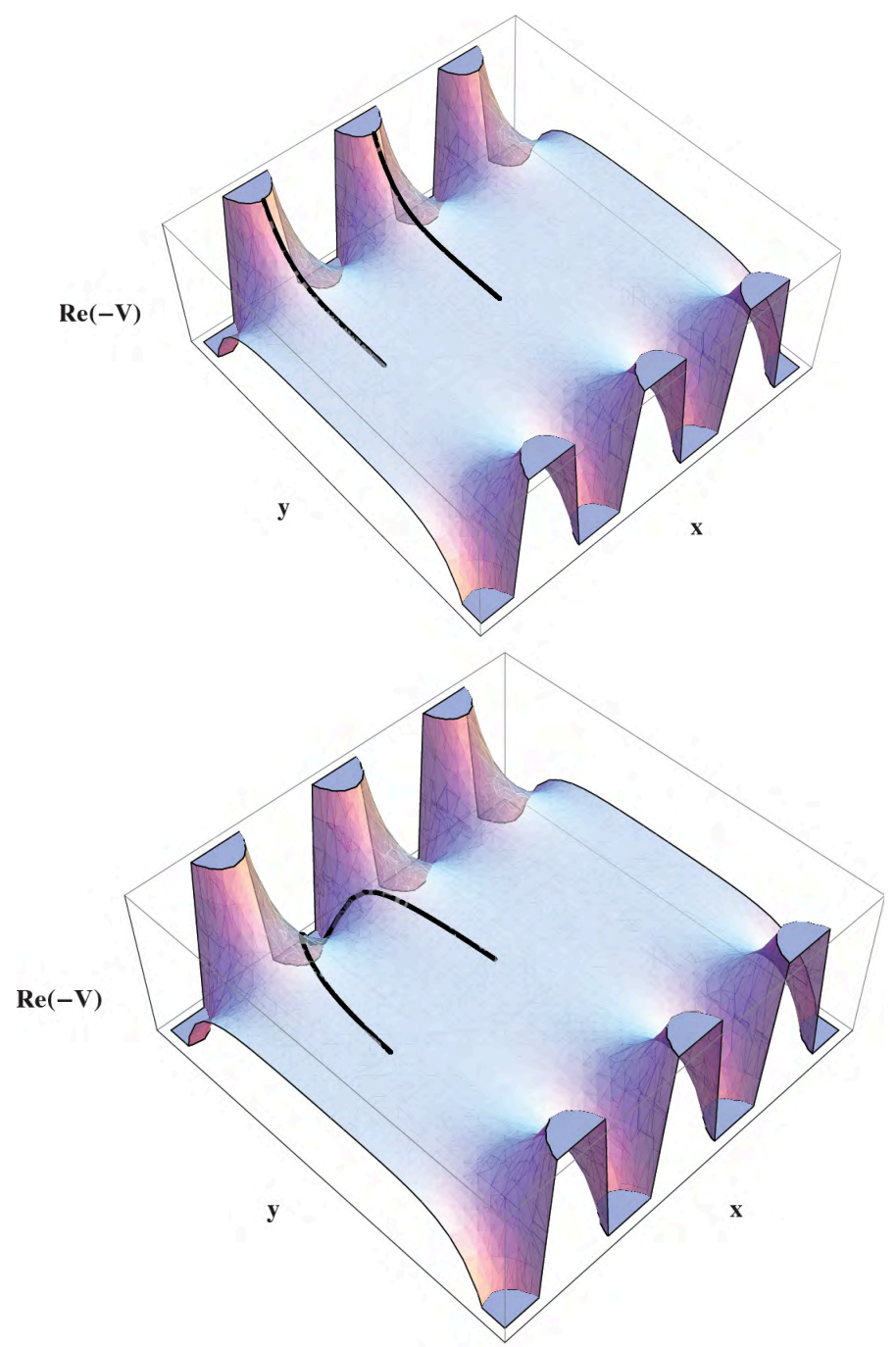

Figure 27. First figure: Complex bion rolling on the real part of the inverted potential for the Sine-Gordon case with $a=1$ and $p g=1 / 10$. The particle begins at $t=0$ at the complex turning point and rolls out along the ridge at $\operatorname{Re}(z)=2 \pi$, reaching the point at infinity in finite time, and then rolls back along the ridge at $\operatorname{Re}(z)=0$, reaching the critical point at $z=0$ at $t= \pm \infty$. The discontinuous jump occurs at infinity. The second figure shows this curve as well as the real bion with $p \rightarrow p e^{-i 19 \pi / 20}$, which effectively smooths out the trajectory so that it passes from one ridge to another in a completely regular way. As $p \rightarrow-p$ we obtain the discontinuous complex bion trajectory.

the holomorphic classical mechanics. Since the motion is constrained by constancy of energy, set to be equal to the energy at one of the turning points (which is on the same critical orbit with a critical point), initializing the motion on the critical orbit to which 
turning point belongs will necessarily result in the particle ending its motion at one of the critical points with this energy.

However, if the potential of motion possesses an exact symmetry under which turning points remains invariant, then the path of the motion must also remain invariant (since the turning point determines the path uniquely). On the other hand, if the critical points are not invariant under this symmetry, but instead go into each other, then the motion cannot connect smoothly to them. This is similar to the paradox of Buridan's donkey. There are two critical points on the same energy level, equidistant from the turning point, and both are equally attractive. The only option is for the motion to evolve to infinity. If the situation is slightly perturbed (i.e. by changing the potential) so that the symmetry in question is broken even by a very small-amount, the motion will reduce to the generic situation and the Euclidean particle will asymptote to one of the critical points. This is precisely what causes the multivaluedness of the solution, i.e. the trajectory depends on how exactly we perturb the potential. The singular nature of the solution also goes hand in hand with multivaluedness, as the only way the particle can move is along a trajectory which obeys the invariance under the symmetry. In the absence of this invariance for critical points, the particle is forced to move off to infinity. This is the singular behavior of the solution.

Example: Let us consider the double-Sine-Gordon case. (Set $a=1$ momentarily.) The real part of the Lagrangian is:

$$
\operatorname{Re} \mathcal{L}=\frac{1}{2}\left(\dot{x}^{2}-\dot{y}^{2}\right)-2 \cos ^{2}\left(\frac{x}{2}\right) \cosh ^{2}\left(\frac{y}{2}\right)+2 \sin ^{2}\left(\frac{x}{2}\right) \sinh ^{2}\left(\frac{y}{2}\right)-\frac{p g}{2} \cos \left(\frac{x}{2}\right) \cosh \left(\frac{y}{2}\right),
$$

and the holomorphic equations of motion, written in terms of real and imaginary part of $z(t)=x(t)+i y(t)$, take the form:

$$
\begin{aligned}
& \frac{d^{2} x}{d t^{2}}=\cosh y \sin x+\frac{p g}{4} \cosh (y / 2) \sin (x / 2), \\
& \frac{d^{2} y}{d t^{2}}=\cos x \sinh y+\frac{p g}{4} \cos (x / 2) \sinh (y / 2) .
\end{aligned}
$$

The transformation

$$
x(t) \rightarrow-x(t)+4 \pi a, \quad y(t) \rightarrow y(t)
$$

is a symmetry of the real part of the holomorphic Lagrangian, as well as the equations of motion. For example, the two turning points located at

$$
z_{ \pm}^{T}=2 \pi a \pm 2 i a \operatorname{arccosh}\left(1+\frac{p g}{4 a^{3}}\right)
$$


go back to themselves upon the transformation (6.54), while the critical point at $z^{\text {cr }}=0$ goes to $z^{\text {cr }}=4 \pi$, and vice versa.

Since only the real part of the potential enters the equations of motion (6.53), if a particle is initialized at the point which obeys the symmetry, then, by uniqueness of the solution, the entire trajectory must obey it. Therefore the Euclidean particle initialized at one of the turning points $z_{k, \pm}^{T}$ cannot asymptote to one of the critical points $z^{\mathrm{cr}}=0$ or $4 \pi a$, but must fly off to infinity.

However if the system is slightly perturbed, for example by giving $p$ a small complex part, $p \rightarrow p e^{i \theta}$, the invariance of the turning points $z_{ \pm}^{T}$ and invariance of the Lagrangian and equations of motion (see (1.20)) is destroyed, and the motion will generically asymptote to one of the critical points. In the limit of real $p$, the motion will turn into the singular motion from the turning point to one of the critical points, depending how the limit of real $p$ is taken. This $i s$ the multivaluedness of the complex bion.

\subsection{Vacuum as a complex and real bion gas}

Reminder of the bosonic case, ground state wave function vs. instanton gas: In the bosonic periodic potential the Euclidean description of the ground state is given by a dilute gas of instantons (at leading order in semi-classical expansion). The ground state wave function is periodic and symmetric. For comparison with the case of a particle with spin, consider a the bosonic potential which is periodic with period $2 \pi a$, but the theory is considered on a circle with circumference $4 \pi a$. Then the ground state is symmetric and the first excited state is anti-symmetric in the interval $[0,4 \pi]$. This is equivalent to the Bloch wave boundary condition $\Psi(x+2 \pi a)=e^{i \alpha} \Psi(x)$, with $\alpha=0$ for the ground state, the lower edge of the band, and $\alpha=\pi$ for the upper edge of the band. The non-perturbative splitting between the first excited state and

the ground state is due to instantons, $\Delta E=J_{\tau_{0}}\left[\frac{\operatorname{det}^{\prime} M}{\operatorname{det} M_{0}}\right]^{-\frac{1}{2}} e^{-S_{\mathcal{I}}}$. The probability to find the particle either at $x=0$ or $x=2 \pi a$ is equal. The dilute instanton gas reflects this property. The classical particle spends half time at $x=0$ and the other half at $x=2 \pi a$. We see that in the theory of a particle with spin (or $N_{f} \geq 1$ ) the ground state structure is dramatically different.

Ground state wavefunction vs. bion gas: Now consider $N_{f} \geq 1$. We have shown that real and complex bions are exact saddles points of the path integral for theories with fermions. The vacuum is two-fold degenerate. The Euclidean description of either one of the vacua is given by a dilute gas of real and complex bions, see Figure 28. The density of both types of bions is $e^{-S_{b}} \approx e^{-2 S_{I}}$. The semi-classical picture is based on a particle that starts at 0 (or an even site) on the left, and makes occasional excursions to 


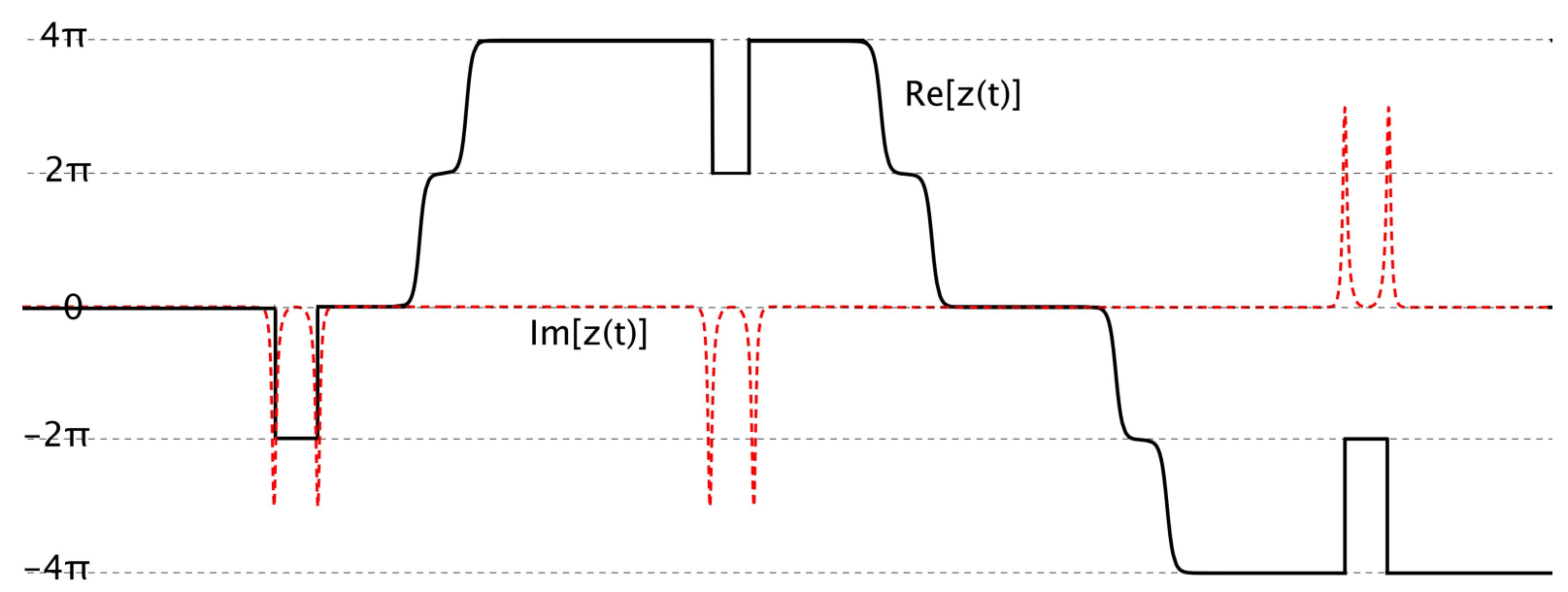

Figure 28. The ground state properties of the theory with fixed number of fermions $\left(N_{f} \geq 1\right)$ is dictated by a dilute gas of real (smooth) and complex (singular) bion events, both with density $e^{-2 S_{I}}$. Both real and complex bions are exact solutions, and Re $\left[2 t_{0}\right]^{\mathrm{cb}}=\left[2 t_{0}\right]^{\mathrm{rb}}$. Note that the Euclidean time spend on $x=2 \pi(\bmod 4 \pi)$ is exponentially small compared to the time spent at $x=0(\bmod 4 \pi)$ by a factor $e^{-2 S_{I}}$.

$2 \pi$ (odd site), where it spends a time $2 t_{0} \approx m_{b}^{-1} \ln \frac{32 a^{3}}{p g} \equiv \tau^{*}$, and immediately returns back to an even site, either back to 0 (complex bion) or forward to $4 \pi$. In other words, the Euclidean particle spends an exponentially short time (unlike the bosonic case) on odd sites. In the wave function language, the wave function must be completely dominated at even sites. The ratio of the probability to find the particle on an odd-site compared to an even site is exponentially small and equal to the bion density:

$$
\frac{\operatorname{Pr} .\left(4 \pi\left(k+\frac{1}{2}\right)\right)}{\operatorname{Pr} .(4 \pi)} \sim e^{-S_{b}} \sim e^{-2 S_{0}} \quad \text { for one of the ground states. }
$$

For the other ground state, odd and even sites are interchanged.

This has a simple interpretation in the Hamiltonian formulation as well. Consider for simplicity the supersymmetric case $N_{f}=1$. The qualitative picture is same for any $N_{f} \geq 1$. The ground state wave functions are given by,

$$
\left\langle x \mid \mathrm{G}_{1}\right\rangle=N e^{+W(x) / g}|\downarrow\rangle, \quad\left\langle x \mid \mathrm{G}_{2}\right\rangle=N e^{-W(x) / g}|\uparrow\rangle .
$$




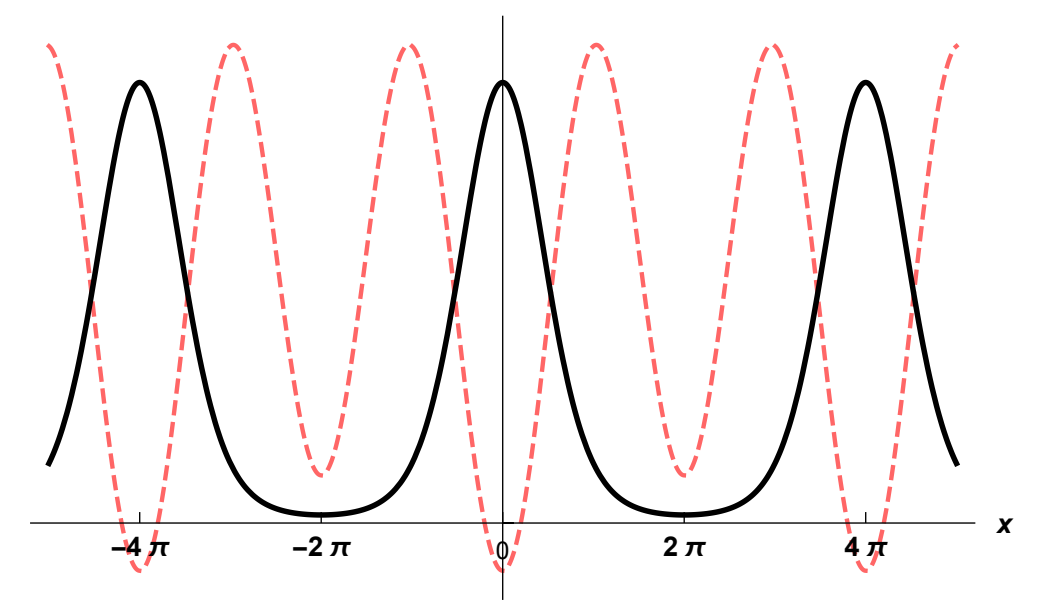

Figure 29. Quantum modified potential (red dashed) for $N_{f} \geq 1$ theories, and associated ground state wave function. The probability to find the particle on an odd-numbered well is suppressed exponentially, by $e^{-2 S_{I}}$, consistent with the dilute bion gas description. As is well-known, in the bosonic case, $N_{f}=0$, the wave function is equally dominant both on the even and odd sites. Note the absence of a bump in the wave function at $x=2 \pi$.

Both \pm states are renormalizable on the interval $[0,4 \pi]$. Consider the + solution. Indeed, this wave function is dominated by even-integer wells, and suppressed at the odd-integer wells:

$$
\frac{\operatorname{Pr} .(2 \pi)}{\operatorname{Pr} .(0)}=\left|\frac{\left\langle 2 \pi \mid \mathrm{G}_{1}\right\rangle}{\left\langle 0 \mid \mathrm{G}_{1}\right\rangle}\right|^{2}=e^{-2 \Delta W / g}=e^{-S_{b}}=e^{-16 a^{3} / g} .
$$

The picture in terms of complex and real bions shown in Figure 28 exactly matches the Hamiltonian picture.

This simple system shows that introducing Grassmann valued fields into the Lagrangian dramatically alters the ground state structure. This change in the ground state structure is the simple quantum mechanics realization of the magnetic bion mechanism in QCD $(\operatorname{adj})[1,3]$, where introducing the Grasssmann valued fermion fields alter the ground state structure in a similar manner with respect to purely bosonic case of deformed Yang-Mills [74, 75].

What would happen if the multi-valued singular saddle did not contribute? Consider the $N_{f}=1$ theory, corresponding to $\mathcal{N}=1$ supersymmetric QM. In this example, it is known that supersymmetry is unbroken. The Witten index $I_{W}=0$, but there are Bose-Fermi degenerate two ground states, (6.57), with $E_{\text {gr }}=0$. The effect of the real bion is to reduce the ground state energy by an amount proportional to $-e^{-2 S_{I}}$. If this was the only contribution there would be a contradictions with supersymmetry algebra, which demands positive semi-definiteness of the ground state energy. However, 

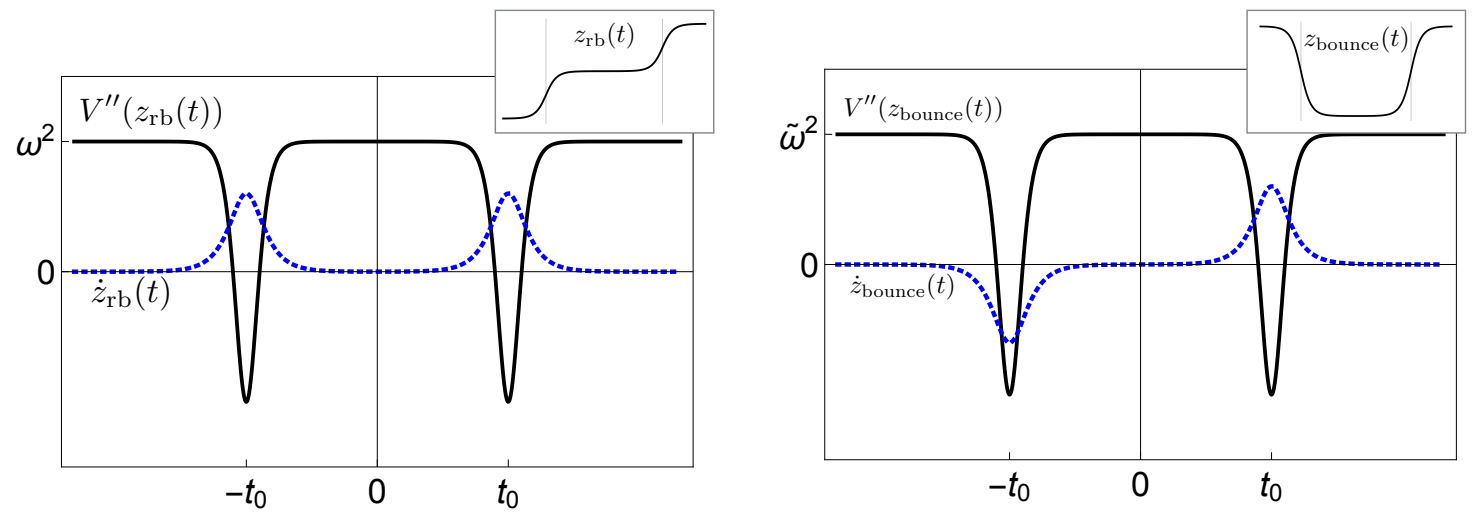

Figure 30. The potential of fluctuation operators for the exact flat-bounce solution and real bion solution.

we showed that there is also a complex bion solution, which gives a contribution of the same magnitude but opposite sign, $E_{\mathrm{gr}} \propto-\left.e^{-2 S_{I} \pm i N_{f} \pi}\right|_{N_{f}=1}=e^{-2 S_{I}}>0$. This is the mechanism for the non-perturbative vanishing of the ground state energy:

$$
E_{\mathrm{gr}} \propto-e^{-2 S_{I}}-e^{-2 S_{I} \pm i \pi}=0 .
$$

Here, as in the double-well problem, $e^{i \pi}$ is the $\mathbb{Z}_{2}$ valued hidden topological angle associated with the complexity of the solution [26].

\subsection{Remark on fluctuation operators}

In the present work, although we do not examine the fluctuation operators around the new exact saddle points in detail, it is worthwhile to point out some of their interesting properties. Consider the fluctuation operators for the bounce and real bion solution. For the complex bion the analysis can either be done by using analytic continuation from the real bounce, or by studying the complex fluctuation operator directly.

The fluctuation operator around a classical solution $z_{\mathrm{cl}}$ is given by

$$
\mathbf{M}=-\frac{d^{2}}{d t^{2}}+\left.V^{\prime \prime}(z)\right|_{z=z_{\mathrm{cl}(t)}} .
$$

Figure 30 depicts the case of the real bion and bounce solutions. The characteristic feature in both cases is the plateau and the double-well structure. This implies that the fluctuation operator differs from the one in the case of instantons, which is a single-well (Pöschl-Teller type), or the one for a typical bounce, which is also usually single-well. Real bion: For the real bion, the zero mode wave function is

$$
\Psi_{0}(t)=\dot{z}_{\mathrm{rb}}(t) .
$$


Since the fluctuation operator $\mathbf{M}$ commutes with parity, $[\mathbf{M}, \mathbf{P}]=0$, the ground state must be parity even. Since $\Psi_{0}(-t)=\Psi_{0}(t)$ and it is nodeless, the ground state wave function of the fluctuation operator is the one of the zero mode wave-function

$$
\Psi_{G}(t)=\Psi_{0}(t), \quad e_{G}=e_{0}=0 .
$$

This is an exact zero mode. In fact, the fluctuation operator is factorizable and hence, a partner in a supersymmetric pair.

The parity-odd anti-symmetric combination is a parametrically small eigenmode of the fluctuation operator, and requires special care. Let us call it $\Psi_{1}(t)$. The energy $e_{1}$ of the $\Psi_{1}(t)$ must be parametrically small with respect to the rest of the spectrum of M. A way to see this is to realize that the parametric smallness of $e_{1}$ itself is an instanton effect. But this instanton is extremely exotic, it actually lives in the fluctuation operator of the real bion solution. So, we call this instanton an "f-instanton". The non-perturbative splitting $e_{1}-e_{0}=e_{1}$ is proportional to $e^{-S_{f I}}$. The f-instanton is a configuration which interpolates between one maximum of $-\left.V^{\prime \prime}(z)\right|_{z=z_{\mathrm{cl}}}$ and the other maximum, but with a flat potential in between. Here, we do not discuss the details of the f-instanton, except for pointing out that it accounts for the parametric smallness of the quasi-zero mode eigenvalue of fluctuation operator.

Bounce: For the real bounce the zero mode wave function is

$$
\Psi_{0}(t)=\dot{z}_{\mathrm{bn}}(t) .
$$

But now, the zero mode with energy $e_{0}=0$ is parity odd, $\Psi_{0}(-t)=-\Psi_{0}(t)$ and has a single node. Since $[\mathbf{M}, \mathbf{P}]=0$, the ground state must be parity even and nodeless. Thus, the zero mode cannot be the ground state, $\Psi_{G}(t)$ :

$$
\Psi_{G}(t) \neq \Psi_{0}(t), \quad e_{G}<e_{0}=0 .
$$

Since $\Psi_{0}(t)$ has only one node, there can only be one negative eigenmode, denoted as $\Psi_{G}(t)$, with a negative eigenvalue. $e_{G}$ is a parametrically small, negative eigenmode. Similar to the previous case, the parametric smallness of the eigenvalue $e_{G}$ is due to finstanton effect, the instanton living in the fluctuation operator. A more detailed study of the fluctuation operators, including the complex fluctuation operators, is deferred to future work.

\section{Complex Saddles and Resurgence}

In both the tilted DW and double-SG quantum mechanics example, we have shown that inclusion of the complex saddles prevents a potential discrepancy between semiclassical analysis and the constraints of supersymmetry. In this sense, the necessity of 
the inclusion of these saddles in supersymmetric theory is not in doubt. However, one may still ask a more general question: In a non-supersymmetric theory, what is the guiding principle which instructs us that complex solutions with multi-fold ambiguous actions must be included? In particular, why does multi-valuedness of these saddles not lead to multi-valuedness in the energy spectrum (which must be unambiguous) of the theory? (This second question and its various incarnations in QFT are prime arguments against the physical significance of multi-valued saddles.) In this brief section, we show that the multi-valuedness associated with the amplitudes of the complex bion cancels with the ambiguity in the left/right Borel resummation of the perturbation series. This is a natural implication of resurgence theory, and a realization of Borel-Ecalle (BE)summability [76-78], providing a mechanism for the reality of the resurgent transseries in the physical domain where it indeed has to be real [30, 65, 79]. A general discussion of all-orders cancellations in real trans-series is elegantly described in [79], also see other examples in [80, 81]. Some aspects of resurgence in supersymmetric quantum mechanics from a complex WKB point of view is also discussed in $[82,83]$.

The contribution of the complex bions to the vacuum energy for the tilted-DW system is

$$
\begin{aligned}
\mathcal{E}_{0} & =+\frac{1}{2 \pi}\left(\frac{-g}{16 a^{3}}\right)^{p-1} \Gamma(p) e^{-8 a^{3} / 3 g} \\
& =+\frac{1}{2 \pi}\left(\frac{g}{16 a^{3}}\right)^{p-1}\left(-\cos (p \pi) \Gamma(p) \pm i \frac{\pi}{\Gamma(1-p)}\right) e^{-8 a^{3} / 3 g}
\end{aligned}
$$

which has both real and imaginary (ambiguous) parts. In the second step, we assumed that $p$ is real. Of course, as it stands, this expression is not acceptable on physical grounds.

Consider perturbation theory for the energy spectrum, in particular the ground state energy, for the tilted DW system. Let $\mathcal{E}_{n}(g)$ be the energy of the state with quantum number $n$ ( $n$ may be a collective quantum number) in units of natural frequency (boson mass) $m_{b}$ of the system. Let

$$
\mathcal{E}_{n}(g)=a_{n, 0}+a_{n, 1} g+a_{n, 2} g^{2}+\ldots=\sum_{q=0}^{\infty} a_{n, q} g^{q} .
$$

The large-order behavior for the ground and first few states are derived using the methods of Bender-Wu $[84,85]$ in Ref.[86], and alternatively, using instantons and dispersion relations in [87]. The Bender-Wu recursive method is based on a Hamiltonian approach, and does not rely on instanton calculus. The leading order factorial divergence of the 
perturbative series are $[86,87]$ :

$$
\begin{aligned}
& a_{0, q} \sim-\frac{6^{-p+1}}{2 \pi} \frac{\Gamma(q-p+1)}{\Gamma(1-p)} \frac{1}{\left(2 S_{I}\right)^{q}}, \\
& a_{1, q} \sim-\frac{6^{-p+3}}{2 \pi} \frac{\Gamma(q-p+3)}{\Gamma(2-p)} \frac{1}{\left(2 S_{I}\right)^{q}} .
\end{aligned}
$$

The perturbative series is a formal asymptotic non-alternating series of the form $q ! /\left(2 S_{I}\right)^{q}$. It is not Borel summable, due to singularities on the positive real axis on the Borel plane, but it is left and right Borel summable, giving a two-fold ambiguous result $\mathcal{S}_{ \pm} \mathcal{E}\left(g^{2}\right)$

Remarkably, the ambiguity inherent to the complex saddle and the ambiguity in

the lateral Borel resummation of perturbation theory cancel each other exactly:

$$
\operatorname{Im}\left[\mathcal{S}_{ \pm} \mathcal{E}\left(g^{2}\right)+I_{\mathrm{cb}}^{ \pm}\right]=0
$$

and the non-perturbative contribution to the ground state energy is

$$
\mathcal{E}_{0}^{\text {n.p. }}=-\frac{1}{2 \pi}\left(\frac{g}{16 a^{3}}\right)^{p-1} \Gamma(p) e^{-8 a^{3} / 3 g} \cos (p \pi) .
$$

This presents a mechanism in which the discontinuities in saddle amplitudes can cancel in the sum over different saddle points, despite the fact that different saddles are hierarchical, providing a resolution of a puzzle addressed in [51].

The crucial (and almost paradoxical) point is that the reality of resurgent transseries associated with a physical observable for real physical values of the couplings demands complexification of the path integral, and contribution of complex multivalued saddles.

\section{Comments on the literature}

\subsection{Witten and Harlow-Maltz-Witten on analytic continuation in Chern- Simons and Liouville theory}

Prior to this work, the most serious study of complex, multi-valued, and singular saddle points of the path integral is described in the recent work of Harlow, Maltz, and Witten (HMW) on Liouville theory [51]. First, we would like to emphasize that there are two closely related ideas:

- The analytic continuation of Euclidean path integrals as coupling constants are continued to the complex domain. 
- The complexification of Euclidean path integrals even if the coupling constants are real.

In the following we will try to explain the relationship between HMW and our work:

1) The first idea is the starting point of the recent work of Witten on Chern Simons theory [25], and of Harlow et al. on Liouville theory [51]. These papers emphasize the need to complexify the path integral once one performs analytic continuation of a coupling parameter into an unphysical region of parameter space. In the case of Chern-Simons theory Witten considers analytic continuation to non-integer values of the coupling $k$.

In the present work we show that even for physical values of the parameters, that means for values of the coupling for which a Hilbert space interpretation exists, the path integral still has to be complexified in order to obtain the correct semi-classical expansion.

2) A more complicated issue raised by HMW is the role of singular and multi-valued solutions. HMW find solutions of this type in analytically continued Liouville theory. In the introduction to their paper HMW state

"Rather surprisingly, we have found that allowing ourselves to use the multi-valued "solutions" just mentioned in the semiclassical expansion enables us to account for the asymptotics of the DOZZ formula throughout the full analytic continuation in the $\eta_{i}$. . . We do not have a clear rationale for why this is allowed."

In the present paper we found a simple quantum-mechanical example in which we provide evidence that multi-valued discontinuous solutions with finite action must be included in the semi-classical expansion. In particular, in the SUSY QM case, we showed that the inclusion of singular solutions is needed to achieve consistency between the supersymmetry analysis and the semi-classical expansion.

3) HMW raise a more specific concern related to multi-valued actions. In footnote 33, HMW state that perhaps discontinuities can cancel in the sum over different saddle points. However, they reject this possibility based on the fact that for generic values of the parameters there will be a complex saddle that is parametrically larger than the rest.

We note that a hierarchy of saddle points also occurs in our examples. However, in the context of resurgence theory this is an expected feature of the semi-classical 
expansion. Indeed, consider a dominant and subdominant saddle, and assume the action associated with the sub-dominant saddle is multi-valued. What happens in resurgent expansions is that the ambiguity associated with perturbation theory around the dominant saddle, is of the same order as the subdominant saddle. Consequently, there may in fact be cancellations of the discontinuities between hierarchical saddles. In Sec. 7 , we provided a concrete realization of such cancellations.

Other studies on complexification of path integration, with different physical motivations, are discussed in [88-92].

\subsection{Brezin, Le Guillou, and Zinn-Justin on complex instantons}

Almost four decades ago Brezin, Le Guillou, and Zinn-Justin (BGZ) [93] considered the potential

$$
V(x)=\frac{1}{2} x^{2}-\gamma x^{3}+\frac{1}{2} x^{4}
$$

which, for $\gamma>1$, is the same as our tilted double-well example, and for $\gamma<1$ corresponds to a potential with a unique minimum. $\gamma=0$ is the focus of the Bender-Wu analysis of the large order behavior of perturbation theory.

In the case $\gamma>1$, the analysis of BGZ corresponds to "half" of our real bounce. BGZ find the real solution interpolating from the local maximum of the inverted potential $-V(x)$ to a real turning point. However, the complex (and sometimes multi-valued) configuration that we call the complex bion, which dictates ground state properties, is not considered in [93]. We note that the complex bion corresponds to either a singularity in the Borel plane on $\mathbb{R}^{+}\left(p \neq \mathbb{N}^{+}\right)$, where the complex bion amplitude has a genuine ambiguity with amplitude $e^{-\operatorname{Re} S_{\mathrm{cb}} \pm i p \pi}$, or to a hidden topological angle $\left(p \in \mathbb{N}^{+}\right)$, where the complex bion amplitude is unambiguous. In the second case, the leading singularity in the Borel plane fades away as $p$ approaches a positive integer. The Stokes multiplier of this configuration is non-zero, and contribute crucially to ground state properties.

In the case $\gamma<1 \mathrm{BGZ}$ construct complex instantons, which interpolate between $x=0$ and a complex turning point $z_{T}, z_{T}^{*}$. This type of complex instanton are argued to lead to a Borel summable series, similar to and are associated with singularities in the $\mathbb{C} \backslash \mathbb{R}^{+}$portion of the Borel plane. For example, in the case $\gamma=0$, the associated singularity is on $\mathbb{R}^{-}$and leads to the famous alternating series, found by Bender and $\mathrm{Wu}[84,85]$. In this case, although complex instantons dictate the perturbative result, their Stokes multiplier is zero. If that were not the case, complex instantons would give a pathological, exponentially increasing, contribution to the path integral of the form $e^{A / g}, A>0$, similar to a particular type of complex instantons in QFT [94]. In 
the present work, the complex saddles have nonzero Stokes multipliers, their weight is exponentially suppressed, $e^{-A / g}, A>0$. It is important to realize that generically the complex bion lead to a Borel non-summable series, unlike the complex instantons, and they are crucial for the correct Euclidean description of the ground state physics.

\subsection{Balitsky and Yung on complex quasi-solutions}

Some of the ideas in our work are inspired from the work of Balitsky and Yung (BY) [52]. In a beautiful, and under-appreciated, paper on semi-classical aspects of supersymmetric quantum mechanics BY considered both supersymmetric QM and the deformation of the Yukawa term in (2.2) away from $p=1$, which is the supersymmetric point. The BY deformation corresponds to the two $S U\left(N_{f}\right)$ singlet sub-sectors in our construction with $k=0$ and $k=N_{f}$.

BY constructed, following Zinn-Justin, an approximate real instanton-anti-instanton configuration. This is not an exact solution, but a good approximation to the exact bounce solution in the weak coupling regime. They also constructed the approximate complex solution by using analytic continuation from the approximate bounce solution, following the idea from [93]. BY correctly interpret this solution as governing the ground state properties. However, BY do not obtain the exact complex solutions found here.

\subsection{Other related works}

There are earlier important works in quantum mechanics in which complexification plays some role. The main distinction between our present study and these works is the following. In our work, we have shown that the ground state properties of a generic quantum mechanical system are governed by complex (sometimes even multivalued) saddles. In the quantum mechanical papers mentioned below, the ground state properties are always governed by real saddles, such as instantons, but complex classical solutions become important when considering the entire spectrum, for example the spectral resolvent. Gutzwiller [95, 96] pioneered the idea of summing over all classical solutions in semiclassical expansions of Green's functions and spectral problems. This was further developed by many authors, from a general formulation in [97, 98], to specific analyses of the symmetric double-well [99-101] and periodic potentials [102]. A complex version of WKB analysis of the pure quartic oscillator also requires inclusion of complex semiclassical configurations in addition to the familiar real Bohr-Sommerfeld ones [103-105]. 


\section{Conclusion}

In this work we studied the semiclassical expansion in two classes of quantum mechanical models with fermions, as well as closely related purely bosonic systems with non-degenerate harmonic minima. We showed that correct semi-classical results can only be obtained if the path integral is complexified, and finite action complex solutions are taken into account. This includes solutions that are singular and multi-valued. Our main conclusions are:

- We argued that in quantum mechanical models with multiple Grassmann fields, the natural setting for finding the semi-classical expansion is a graded formulation in which fermions are integrated out. This induces a quantum modified potential for the bosonic fields.

- The real Euclidean equations of motion in the inverted potential may or may not have real finite action solutions. But once the path integral is complexified, the critical points are described by the solutions of the holomorphic Newton equation in the inverted quantum modified potential:

$$
\frac{d^{2} z}{d t^{2}}=+\frac{d V}{d z}=W^{\prime}(z) W^{\prime \prime}(z)+\frac{p g}{2} W^{\prime \prime \prime}(z) .
$$

This equation generically admits complex, multi-valued finite action solutions.

- The characteristic size of the real and complex bion saddle solutions is parametrically larger than the natural size of the instanton solution in the original formulation. The instanton size is parametrically $\omega^{-1}$ where $\omega$ is natural frequency, and bion size is $\tau^{*} \sim \omega^{-1} \ln \frac{A a^{3}}{g p}$. The non-BPS bion solutions are exact for any value of the coupling. In the weak coupling regime, it can be described as a correlated two-instanton event (which is only approximate quasi-solution).

- The action of the exact non-BPS solution is in general multi-valued, and weight of these saddles is of the form $\sim e^{-S_{\mathrm{r}} \pm i p \pi}$. For $N_{f}=1,2, \ldots$, where $p=N_{f}-2 k$, the multi-valuedness of the action disappears since the imaginary part of the action is defined modulo $2 \pi$. This gives the $\mathbb{Z}_{2}$ valued hidden topological angle [26].

- For non-integer values of $p$, the multi-valuedness of the fugacity is canceled by Borel resummation of perturbation theory, an explicit realization of resurgence. In this sense, neither divergence of a series (or its ambiguous Borel resummation), nor the multi-valued saddles are meaningless or a nuisance. Rather, they are essential in order to give proper meaning to the path integral. 
- In generic quantum mechanical systems, the Euclidean description of the vacuum is a dilute gas of complex and real bions. In particular, the much better known instantons, and the dilute instanton gas picture, are relatively rare cases relevant for potentials with symmetry.

\subsection{Prospects in quantum field theories}

In this paper we pursued two strategies for constructing saddles in path integration, one of which is exact, and the other approximate.

(A) Exact solution of complexified Newton equations after integrating out fermions exactly.

(B) Construct BPS solutions, and glue them together by integrating over the complexified quasi-zero mode thimble, $\Gamma^{\mathrm{qzm}}$, using the finite dimensional steepest descent method.

In weak coupling we have shown that all the essential features of the exact solutions, their real or complex nature, multi-valuedness, singular or smooth behavior, hidden topological angle, characteristic size, and monodromy properties, are reproduced by the second (approximate) method. This makes us confident that the second method, (B), is indeed a systematic approximation to exact saddle point calculations. ${ }^{19}$ The physical reason for this conjecture is based on the following argument: Consider a twoinstanton event, and fluctuations around it. The center position of the two instantons is an exact zero mode, the relative position is a quasi-zero mode, and the remaining modes are Gaussian. The quasi-zero mode is special in this sense. An exact integration over the $\Gamma^{\mathrm{qzm}}$-cycle, which passes through the saddle of the interaction potential of the two instantons is capable of producing the features of the exact solution.

The first method, (A), is hard to generalize to QFT. In studying the quantum mechanics of a single particle with spin degrees of freedom we are in a somewhat fortunate situation in the sense that we can write the notorious fermion determinant $N_{f} \log \operatorname{det}(D)$ (where $D$ is the Dirac operator) as a sum of local modifications of the bosonic potential. This is important for constructing of exact solutions. In QFT, this is a formidable task, although in certain background field problems it has been shown, using the world-line representation of the fermion determinant, that complex saddles govern the physics [106].

Another consideration is that in bosonic quantum field theories and quantum mechanics, when the topological theta angle is set to zero, the contribution of instantons

\footnotetext{
${ }^{19}$ Proving this statement starting with the complex gradient flow equations by using techniques from partial differential equations (PDEs) would represent major progress in Picard-Lefschetz theory.
} 
(as well as other real saddles) to the ground state energy is universally negative. In theories with fermions (multiple Grassmann valued fields), instantons do not contribute to the ground state energy because of the fermionic zero modes. At second order, there can be either a negative or positive contribution. In fact, for real saddles (at second order or otherwise), the contribution to the ground state energy is universally negative. For complex saddles, whether the contribution is negative or positive depends on the hidden topological angle. For example, in the present work, we showed that complex saddles contribute to the ground state energy as $-\cos (p \pi) e^{-S_{\mathrm{r}}}$, so it is positive for $p=1$, and negative for $p=2$.

If we accept the idea that the second method, (B), is indeed a systematic approximation to exact saddle points in QFT, we find ourselves in an interesting situation. In particular, such real and complex saddles must exist in four dimensional gauge theories, QCD and SQCD on $\mathbb{R}^{4}, \mathcal{N}=1$ SYM and $\mathrm{QCD}(\operatorname{adj})$ on $\mathbb{R}^{3} \times S^{1}, \mathcal{N}=2 \mathrm{SYM}$ on $\mathbb{R}^{3}$, and sigma models such as $\mathbb{C P}^{N-1}$ and $O(N)$ models with fermions on $\mathbb{R}^{2}$ as well as $\mathbb{R}^{1} \times S^{1}$, and more.

Consider for example $\mathcal{N}=1 \mathrm{SYM}$. It is a vector-like (QCD-like) theory, without an elementary scalar. It is also a minimal supersymmetric gauge theory, and an integral part of all SQCD theories. In this sense, it is a useful model to describe phenomena that may take place both in QCD and SQCD. On $\mathbb{R}^{3} \times S_{1}$, at leading order in the semiclassical expansion, this theory has monopole-instantons which induce a superpotential $[107,108]$. At second order in the semi-classical expansion, the microscopic origin of the bosonic potential can be understood in terms of magnetic bions (in one-to-one correspondence with the positive entries of the extended Cartan matrix) and neutral bions (in one-to-one correspondence with the negative entries of extended Cartan matrix) $[1,14,109]$. For example, for $S U(2)$ gauge theory, the bosonic potential is

$$
V(\phi, \sigma) \sim-e^{-2 S_{m}} \cos (2 \sigma)-e^{-2 S_{m} \pm i \pi} \cosh (2 \phi),
$$

where $\sigma$ is the dual photon and $\phi$ is the fluctuation of the holonomy relative to the center symmetric point. Magnetic bions contribute negatively to the ground state energy (found by setting $\sigma=0, \phi=0$ ), and neutral bions contribute positively:

$$
E_{\text {gr }} \propto-e^{-2 S_{m}}-e^{-2 S_{m} \pm i \pi}=0
$$

Note that this formula is identical to the one we found in supersymmetric quantum mechanics with periodic superpotential (6.59). There is a relative $e^{i \pi}$ hidden topological angle difference between the two amplitudes. According to our criterion, it seems plausible that the magnetic bion is associated with an exact real saddle while the neutral bion is associated with an exact complex saddle. All statements concerning 
two-events (second order semi-classics) in $\mathcal{N}=1 \mathrm{SYM}$ are also valid for QCD(adj) on $\mathbb{R}^{3} \times S^{1}$, as well as non-linear sigma models on $\mathbb{R}^{1} \times S^{1}[17,18,44]$.

Similarly, in three-dimensional $\mathcal{N}=2$ SUSY gauge theory [110], the bosonic potential can either be derived from the superpotential or by performing a quasi-zero mode integration, and provides a positive definite run-away potential,

$$
V(\phi) \sim-e^{-2 S_{m} \pm i \pi} e^{-2 \phi}
$$

The origin of the positive definiteness of the run-away potential is a complex phase that arises from the QZM integration.

Finally, in SQCD with $N_{f}=N_{c}-1$ in $\mathbb{R}^{4}$, the semi-classical analysis is reliable, because the IR-divergence due to the instanton size modulus is cut-off by the scalar meson vacuum expectation value. The theory has a bosonic run-away potential, which can either be derived from the instanton-induced ADS-superpotential [111], or alternatively, it can be derived by computing the $[\mathcal{I} \overline{\mathcal{I}}]$ two-event amplitude $[112]$. In order for the potential to be positive semi-definite, the contribution of the $[\mathcal{I} \overline{\mathcal{I}}]$ saddle must be $-e^{-2 S_{I}+i \pi}$. This also suggest that the $[\mathcal{I} \overline{\mathcal{I}}]$ event in SQCD may in fact be be an exact complex saddle.

Our results suggest that the natural formalism for the semi-classical analysis of quantum field theory, and quantum mechanics, is the complexified version of the path integral. This perspective provides a physical interpretation for non-BPS solutions [113-116], and possibly also for complex instantons [117].

\subsection{Other Related Directions}

Lattice constructions and sign problem: The Lefschetz thimble decomposition is applied to QFTs with sign problems in various lattice theories, see, e.g. [53-56, 118]. Very often, these simulations are run in the vicinity of one saddle. In the present work, we have seen that the effect of different saddles generically have different phases, leading to a (Euclidean version of) interference in the sum over saddles. For example, vanishing of the vacuum energy in supersymmetric theory is of the form $E_{\mathrm{gr}}=0 e^{-S_{\mathrm{pert}}}-$ $e^{-S_{\mathrm{rb}}}-e^{i \pi} e^{-S_{\mathrm{rb}}}$, where $e^{i \pi}$ is the $\mathbb{Z}_{2}$ valued hidden topological angle (HTA), leading to destructive interference among saddles [26, 27]. This important point is also emphasized in the context of sign problem in [119, 120]. Clearly, future lattice (as well as continuum) studies must take into account the sum over all contributing saddles due to interference effects induced by the HTA.

Complex gradient flow equations and $\mathcal{N}=2$ instantons: Readers familiar with extended $(\mathcal{N}=2)$ supersymmetric quantum mechanics will realize that the complex gradient flow equation (1.16) in a $d=0$ dimensional "field theory" is identical to 
the instanton equation for the $\mathcal{N}=2$ system with superpotential $W(z)=f(z)$.

$$
\frac{d z}{d u}=e^{i \theta} \frac{\partial \bar{W}}{\partial \bar{z}}
$$

where $\theta$ in QM may be identified with the phase of the central charge, and $u$ is the gradient flow time (which becomes Euclidean time in the second interpretation). Namely, thimbles in the simple zero dimensional integrals are related to integration cycles in path integrals of $(\mathcal{N}=2)$ supersymmetric QM related to instantons. This also generalizes to certain QFTs, see [25] and the following item. We find this connection intriguing.

Parabolic vs. elliptic gradient flow: The first path integral analyses of the Lefschetz thimble decomposition were performed for Chern-Simons theory and the phase space path integral (where the Hamiltonian is set to zero) [25, 88]. In both cases, the complex gradient flow equations are elliptic PDEs, and possess a higher dimensional (Euclidean version of Lorentz) symmetry, e.g. for Chern-Simons, these equations are

$$
\frac{\partial \mathcal{A}_{i}}{\partial u}=-e^{i \theta} \frac{\delta \overline{\mathcal{S}}_{\mathrm{CS}}}{\delta \overline{\mathcal{A}}_{i}}=\frac{1}{2} e^{i \theta} \epsilon_{i j k} \overline{\mathcal{F}}_{j k}
$$

which is the complex generalization of the instanton equation in $A_{4}=0$ gauge:

$$
\mathcal{F}^{(2)}=e^{i \theta} * \overline{\mathcal{F}}^{(2)} \quad \text { or } \quad \mathcal{F}_{\mu \nu}=e^{i \theta} \frac{1}{2} \epsilon_{\mu \nu \rho \sigma} \overline{\mathcal{F}}_{\rho \sigma}
$$

The reason for the $3 d-4 d$ connection is actually not an accident: Thimbles in $d=3$ dimensional Chern-Simons theory [25] are related to the complexified instanton equations in the twisted version of the $\mathcal{N}=4$ super Yang-Mills theory in four dimensions. Indeed, (9.7) appeared first in the context of lattice supersymmetry [121], and in the Geometric Langlands program [122], again, not accidentally, because both of these are applications of the same twist. Witten argues that the nice properties of the finite dimensional Lefschetz thimble decomposition carry over to the infinite dimensional functional integral for the elliptic flow equations [25]. On the other hand, for almost all interesting QFTs and configuration space path integrals, the complex gradient flow equations are parabolic. It will be interesting to understand more precisely the relation between the nature of the flow equation (parabolic vs. elliptic) and the nature of the saddles (smooth/singular/multivalued), and the implications for the the thimble construction.

Clearly, it is of interest to study general field theories using complexified path integrals. We expect other interesting applications in cosmology, string theory, and condensed matter; indeed in any problem in which the path integral is a useful tool. 


\section{Acknowledgments}

We thank P. Argyres, G. Başar, A. Cherman, O. Costin, D. Dorigoni, D. Harlow, C. Howls, M. Kaminski, K. Konishi, J. Maltz, D. O’ Connor, R. Pisarski, E. Poppitz, R. Riwar, M. Shifman, Y. Tanizaki, B. Tekin, E. Witten, S. T. Yau and A. Yung for useful comments and discussions. M. ̈̈. thanks especially E. Witten for encouragement and probing questions. M.Ü.'s work was partially supported by the Center for Mathematical Sciences and Applications (CMSA) at Harvard University. Part of this work was completed while T.S. was a visitor at the Aspen Center for Physics, which is supported by National Science Foundation grant PHY-1066293. We acknowledge support from DOE grants DE-FG02-03ER41260 (T.S.), DE-SC0013036 (M.U.) and DE-SC0010339 (G.D.).

\section{A Review of some relevant elliptic integrals}

In this appendix, we summarize some basic result concerning elliptic functions and elliptic integrals [71]. These result are used in constructing the exact bounce and bion solutions. Let

$$
t=\int_{z_{a}}^{z} \frac{d w}{\sqrt{F(w)}}
$$

where $F(w)$ is a quartic polynomial with no repeated factors

$$
F(w):=a_{4} w^{4}+4 a_{3} w^{3}+6 a_{2} w^{2}+4 a_{1} w+a_{0}
$$

Then, the general solution to

$$
\ddot{z}=\frac{\partial F}{\partial z}
$$

is given by

$$
z(t)=z_{a}+\frac{\sqrt{F\left(z_{a}\right)} \wp^{\prime}\left(t ; g_{2}, g_{3}\right)+\frac{1}{2} F^{\prime}\left(z_{a}\right)\left[\wp\left(t ; g_{2}, g_{3}\right)-\frac{1}{24} F^{\prime \prime}\left(z_{a}\right)\right]+\frac{1}{24} F\left(z_{a}\right) F^{\prime \prime \prime}\left(z_{a}\right)}{2\left[\wp\left(t ; g_{2}, g_{3}\right)-\frac{1}{24} F^{\prime \prime}\left(z_{a}\right)\right]^{2}-\frac{1}{48} F\left(z_{a}\right) F^{\prime \prime \prime \prime}\left(z_{a}\right)},
$$

where $\wp\left(t ; g_{2}, g_{3}\right)$ is the Weierstrass elliptic function with algebraic quadratic and cubic lattice invariants $g_{2}$ and $g_{3}$ related to the coefficients of the quartic polynomial $F$ in (A.2) by

$$
\begin{aligned}
& g_{2}:=a_{4} a_{0}-4 a_{3} a_{1}+3 a_{2}^{2} \\
& g_{3}:=a_{4} a_{2} a_{0}-4 a_{3} a_{2} a_{1}-a_{2}^{3}-a_{4} a_{1}^{2}-a_{3}^{2} a_{0} .
\end{aligned}
$$


The function $\wp\left(t ; g_{2}, g_{3}\right)$ is an even, doubly-periodic, elliptic function with periods $T_{1}$ and $T_{2}$ expressed in terms of the invariants $g_{2}, g_{3}$ of the associated algebraic curve of genus $g=1$ :

$$
g_{2}=60 \sum_{(m, n) \neq(0,0)}\left(m T_{1}+n T_{2}\right)^{-4}, \quad g_{3}=140 \sum_{(m, n) \neq(0,0)}\left(m T_{1}+n T_{2}\right)^{-6} .
$$

The complex periods $T_{1}$ and $T_{2}$ define a $2 \mathrm{D}$ lattice, and $\wp$ parametrizes the algebraic curve $F(z)$ corresponding to a complex Riemann surface with genus $g=1$; i.e., a torus with two periods corresponding to each cycle $S^{1}$. More technically, this torus is obtained by ramifying a Riemann sphere at 4 points, i.e., zeros of $F(z)$, whose branched double covering is the function $\wp$. In the generalization of our solutions to higher degree potentials, the energy conservation relation defines a higher genus complex algebraic curve, and the role of the Weierstrass function is played by hyperelliptic and automorphic functions.

If $z_{a}$ is a zero of $F(z)$, which corresponds to the relevant situation of $z_{a}$ being a turning point, then the general solution (A.4) simplifies significantly to:

$$
z(t)=z_{a}+\frac{\frac{1}{4} F^{\prime}\left(z_{a}\right)}{\wp(t)-\frac{1}{24} F^{\prime \prime}\left(z_{a}\right)} .
$$

This is our "master formula", which gives the general solution to the Euclidean equations of motion. To find bounce or bion solutions which pass through certain physically important turning points and critical points, we must specify the point $z_{a}$ and determine the appropriate invariants $g_{2}$ and $g_{3}$. Note that the "Euclidean energy" $E$ in (5.8) enters into the quartic polynomial $F(z) \equiv 2(E+V(z))$, so the invariants $g_{2}$ and $g_{3}$ depend on $E$, and take especially simple values when $z_{a}$ is a critical point of the potential.

Note the interesting but elementary algebraic fact that for a quartic polynomial $F(z)$, as in (A.2), if $z_{1}$ and $z_{2}$ are two points such that $F\left(z_{1}\right)=F\left(z_{2}\right)$, then

$$
F^{\prime}\left(z_{1}\right)+F^{\prime}\left(z_{2}\right)=\frac{1}{6}\left(z_{1}-z_{2}\right)\left(F^{\prime \prime}\left(z_{1}\right)-F^{\prime \prime}\left(z_{2}\right)\right) .
$$

\section{B Absence of a Hidden Topological Angle in one-dimensional real integrals with real parameters}

Consider a one dimensional integral

$$
I=\int_{-\infty}^{\infty} d x e^{-\frac{1}{\hbar} S(x)} .
$$


with $S(x)$ real for any $x \in \mathbb{R}$, and $\hbar>0$. Consider decomposing the real integration cycle $\mathbb{R}$ into a sum over Lefschetz thimbles: $\mathbb{R}=\sum_{\sigma} n_{\sigma} \mathcal{J}_{\sigma}$. Here, we prove that if $z_{\sigma}$ is a contributing saddle to the integration, i.e, $n_{\sigma} \neq 0$, then the phase associated with this cycle must be zero. This proves that the HTA phenomenon that we found in the semi-classical analysis of the path integral over real fields and real values of the parameters does not have a counterpart in one-dimensional exponential type integrals, and does not provide an accurate intuition for the problem. This may be one reason why the possibility of complex saddles contributing to the semi-classical analysis of path integrals was not taken sufficiently seriously in the past.

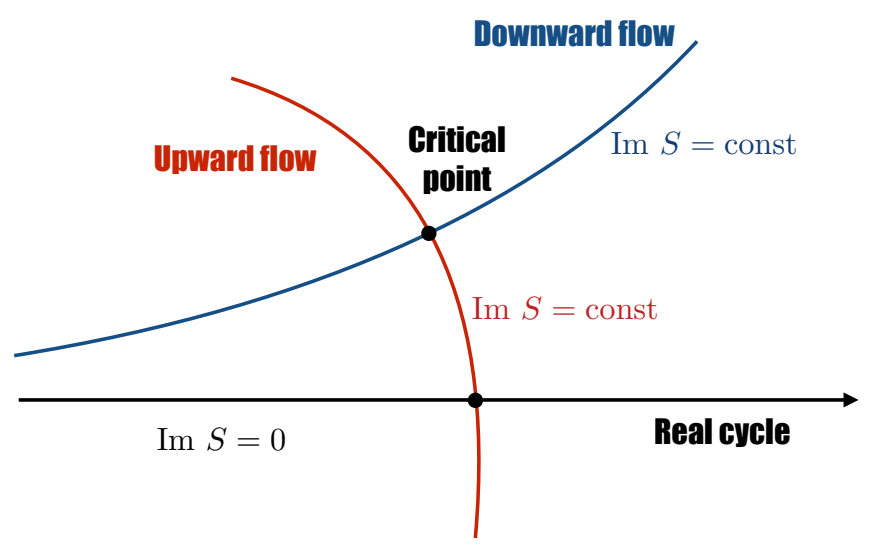

Figure 31. Sketch of upward and downward flows from a critical point.

If the integral has a critical point at $z_{\sigma}$, then the steepest descent equation $\operatorname{Im} S(z)=$ $\operatorname{Im} S\left(z_{\sigma}\right)$ has two solutions: "the upward flow" $\mathcal{J}_{\sigma}$, and the "downward flow" $\mathcal{K}_{\sigma}$ (see Fig. 31). The "upward flow" ("downward flow") is the solution under which Re $(-S(z))$ increases (decreases) away from the critical point. For the cycle $\mathcal{J}_{\sigma}$ to contribute to the cycle decomposition of the real line $\mathbb{R}$, its upward flow $\mathcal{K}_{\sigma}$ must intersect the real line. However since $\operatorname{Im} S(x)=0, \forall x \in \mathbb{R}$, and since the phase is stationary over the ascent thimble, $\operatorname{Im} S(x)=$ const, $\forall z \in \mathcal{K}_{0}$, it follows that $\operatorname{Im} S\left(z_{\sigma}\right)=0$. This shows that HTA of any critical point which contributes to the integral (B.1) is always identically zero.

\section{References}

[1] M. Ünsal, Magnetic bion condensation: A New mechanism of confinement and mass gap in four dimensions, Phys.Rev. D80 (2009) 065001, [arXiv:0709.3269]. 
[2] E. Poppitz and M. Ünsal, Seiberg-Witten and 'Polyakov-like' magnetic bion confinements are continuously connected, JHEP 1107 (2011) 082, [arXiv:1105.3969].

[3] M. M. Anber and E. Poppitz, Microscopic Structure of Magnetic Bions, JHEP 1106 (2011) 136, [arXiv:1105.0940].

[4] T. Misumi and T. Kanazawa, Adjoint $Q C D$ on $\mathbb{R}^{3} \times S^{1}$ with twisted fermionic boundary conditions, JHEP 1406 (2014) 181, [arXiv: 1405.3113].

[5] S. R. Coleman, The Uses of Instantons, Subnucl.Ser. 15 (1979) 805.

[6] J. Zinn-Justin, Quantum field theory and critical phenomena, Int.Ser.Monogr.Phys. 113 (2002) 1-1054.

[7] V. A. Rubakov, Classical theory of gauge fields. Princeton, USA: Univ. Pr. (2002) 444 p, 2002.

[8] X. G. Wen, Quantum field theory of many-body systems: From the origin of sound to an origin of light and electrons. Oxford, 2004.

[9] A. Altland and B. Simons, Condensed matter field theory. Cambridge University Press, 2006.

[10] E. J. Weinberg, Classical solutions in quantum field theory. Cambridge Monographs on Mathematical Physics. Cambridge University Press, 2012.

[11] T. Schäfer and E. V. Shuryak, Instantons in QCD, Rev.Mod.Phys. 70 (1998) 323-426, [hep-ph/9610451].

[12] N. Dorey, T. J. Hollowood, V. V. Khoze, and M. P. Mattis, The Calculus of many instantons, Phys. Rept. 371 (2002) 231-459, [hep-th/0206063].

[13] S. Vandoren and P. van Nieuwenhuizen, Lectures on instantons, arXiv:0802.1862.

[14] P. C. Argyres and M. Ünsal, The semi-classical expansion and resurgence in gauge theories: new perturbative, instanton, bion, and renormalon effects, JHEP 1208 (2012) 063, [arXiv:1206.1890].

[15] P. Argyres and M. Ünsal, A semiclassical realization of infrared renormalons, Phys.Rev.Lett. 109 (2012) 121601, [arXiv:1204.1661].

[16] G. V. Dunne and M. Ünsal, Continuity and Resurgence: towards a continuum definition of the CP(N-1) model, Phys.Rev. D87 (2013) 025015, [arXiv:1210.3646].

[17] G. V. Dunne and M. Ünsal, Resurgence and Trans-series in Quantum Field Theory: The CP(N-1) Model, JHEP 1211 (2012) 170, [arXiv:1210.2423].

[18] A. Cherman, D. Dorigoni, G. V. Dunne, and M. Ünsal, Resurgence in Quantum Field Theory: Nonperturbative Effects in the Principal Chiral Model, Phys.Rev.Lett. 112 (2014), no. 2 021601, [arXiv:1308.0127]. 
[19] M. V. Fedoryuk, The saddle-point method, Izdat. "Nauka," Moscow, MR 58:22580 (1977).

[20] D. Kaminski, Exponentially improved stationary phase approximations for double integrals, Methods Appl. Anal. 1, 44 - 56. MR 95i:41056 (1994).

[21] F. Pham, Vanishing homologies and the $n$ variable saddlepoint method, Proc. Symp. Pure Math 2 (1983), no. 40 319-333.

[22] M. V. Berry and C. J. Howls, Hyperasymptotics for integrals with saddles, Proceedings of the Royal Society of London A: Mathematical, Physical and Engineering Sciences 434 (1991), no. 1892 657-675.

[23] C. J. Howls, "hyperasymptotics for multidimensional integrals, exact remainder terms and the global connection problem,", Proc. R. Soc. London, 453 (1997) 2271.

[24] E. Delabaere and C. J. Howls, Global asymptotics for multiple integrals with boundaries, Duke Math. J. 112 (04, 2002) 199-264.

[25] E. Witten, Analytic Continuation Of Chern-Simons Theory, AMS/IP Stud. Adv. Math. 50 (2011) 347-446, [arXiv:1001.2933].

[26] A. Behtash, T. Sulejmanpasic, T. Schäfer, and M. Ünsal, Hidden Topological Angles in Path Integrals, Phys. Rev. Lett. 115 (2015), no. 4 041601, [arXiv:1502.0662].

[27] A. Behtash, E. Poppitz, T. Sulejmanpasic, and M. Ünsal, The curious incident of multi-instantons and the necessity of Lefschetz thimbles, arXiv:1507.0406.

[28] E. Bogomolny, Calculation of instanton-anti-instanton contributions in quantum mechanics, Phys.Lett. B91 (1980) 431-435.

[29] J. Zinn-Justin, Multi - Instanton Contributions in Quantum Mechanics, Nucl.Phys. B192 (1981) 125-140.

[30] G. Basar, G. V. Dunne, and M. Ünsal, Resurgence theory, ghost-instantons, and analytic continuation of path integrals, JHEP 10 (2013) 041, [arXiv:1308.1108].

[31] M. Kontsevich, Resurgence from the path integral perspective, Talk at Perimeter Institute, (2012).

[32] M. Kontsevich, Exponential integrals, Talks at Simons Center and at IHES (2014, 2015).

[33] M. Kontsevich, On non-perturbative quantization, fukaya categories and resurgence, Talk at Simons Center (2015).

[34] M. Adler and P. van Moerbeke, The complex geometry of the kowalewski-painleve analysis, Inventiones mathematicae 97 (1989), no. 1 3-51.

[35] E. K. Sklyanin, Quantum inverse scattering method. Selected topics, hep-th/9211111. 
[36] N. A. Nekrasov and S. L. Shatashvili, Quantization of Integrable Systems and Four Dimensional Gauge Theories, in Proceedings, 16th International Congress on Mathematical Physics (ICMP09), 2009. arXiv:0908.4052.

[37] N. A. Nekrasov and S. L. Shatashvili, Quantum integrability and supersymmetric vacua, Prog. Theor. Phys. Suppl. 177 (2009) 105-119, [arXiv:0901.4748].

[38] K. K. Kozlowski and J. Teschner, TBA for the Toda chain, arXiv:1006.2906.

[39] G. V. Dunne and M. Ünsal, Generating Non-perturbative Physics from Perturbation Theory, Phys.Rev. D89 (2014) 041701, [arXiv:1306.4405].

[40] G. V. Dunne and M. Ünsal, Uniform WKB, Multi-instantons, and Resurgent Trans-Series, Phys.Rev. D89 (2014), no. 10 105009, [arXiv:1401.5202].

[41] T. Misumi, M. Nitta, and N. Sakai, Resurgence in sine-Gordon quantum mechanics: Exact agreement between multi-instantons and uniform WKB, arXiv:1507.0040.

[42] M. A. Escobar-Ruiz, E. Shuryak, and A. V. Turbiner, Three-loop Correction to the Instanton Density. I. The Quartic Double Well Potential, Phys. Rev. D92 (2015), no. 2 025046, [arXiv:1501.0399].

[43] M. A. Escobar-Ruiz, E. Shuryak, and A. V. Turbiner, Three-loop Correction to the Instanton Density. II. The Sine-Gordon potential, Phys. Rev. D92 (2015), no. 2 025047, [arXiv: 1505.0511].

[44] A. Cherman, D. Dorigoni, and M. Ünsal, Decoding perturbation theory using resurgence: Stokes phenomena, new saddle points and Lefschetz thimbles, arXiv: 1403.1277.

[45] T. Misumi, M. Nitta, and N. Sakai, Neutral bions in the $\mathbb{C} P^{N-1}$ model, JHEP 1406 (2014) 164, [arXiv:1404.7225].

[46] T. Misumi, M. Nitta, and N. Sakai, Classifying bions in Grassmann sigma models and non-Abelian gauge theories by D-branes, PTEP 2015 (2015) 033B02, [arXiv: 1409.3444].

[47] M. Nitta, Fractional instantons and bions in the $O(N)$ model with twisted boundary conditions, JHEP 03 (2015) 108, [arXiv:1412.7681].

[48] E. Witten, Dynamical Breaking of Supersymmetry, Nucl.Phys. B188 (1981) 513.

[49] E. Witten, Constraints on Supersymmetry Breaking, Nucl.Phys. B202 (1982) 253.

[50] V. Arnold, Mathematical methods of classical mechanics, vol. 60. Springer, 1989.

[51] D. Harlow, J. Maltz, and E. Witten, Analytic Continuation of Liouville Theory, JHEP 1112 (2011) 071, [arXiv:1108.4417].

[52] I. Balitsky and A. Yung, Instanton Molecular Vacuum in $N=1$ Supersymmetric Quantum Mechanics, Nucl.Phys. B274 (1986) 475. 
[53] AuroraScience Collaboration Collaboration, M. Cristoforetti, F. Di Renzo, and L. Scorzato, New approach to the sign problem in quantum field theories: High density QCD on a Lefschetz thimble, Phys.Rev. D86 (2012) 074506, [arXiv:1205.3996].

[54] M. Cristoforetti, F. Di Renzo, A. Mukherjee, and L. Scorzato, Monte Carlo simulations on the Lefschetz thimble: Taming the sign problem, Phys.Rev. D88 (2013), no. 5 051501, [arXiv:1303.7204].

[55] H. Fujii, D. Honda, M. Kato, Y. Kikukawa, S. Komatsu, et. al., Hybrid Monte Carlo on Lefschetz thimbles - A study of the residual sign problem, JHEP 1310 (2013) 147, [arXiv:1309.4371].

[56] G. Aarts, L. Bongiovanni, E. Seiler, and D. Sexty, Some remarks on Lefschetz thimbles and complex Langevin dynamics, JHEP 1410 (2014) 159, [arXiv:1407.2090].

[57] T. Kanazawa and Y. Tanizaki, Structure of Lefschetz thimbles in simple fermionic systems, JHEP 03 (2015) 044, [arXiv: 1412.2802].

[58] Y. Tanizaki, Lefschetz-thimble techniques for path integral of zero-dimensional $O(n)$ sigma models, Phys. Rev. D91 (2015), no. 3 036002, [arXiv:1412.1891].

[59] H. Nishimura, M. C. Ogilvie, and K. Pangeni, Complex saddle points in QCD at finite temperature and density, Phys. Rev. D90 (2014), no. 4 045039, [arXiv:1401.7982].

[60] H. Nishimura, M. C. Ogilvie, and K. Pangeni, Complex Saddle Points and Disorder Lines in QCD at finite temperature and density, Phys. Rev. D91 (2015), no. 5 054004, [arXiv: 1411.4959].

[61] Y. Tanizaki and T. Koike, Real-time Feynman path integral with Picard-Lefschetz theory and its applications to quantum tunneling, Annals Phys. 351 (2014) 250-274, [arXiv:1406.2386].

[62] A. Cherman and M. Ünsal, Real-Time Feynman Path Integral Realization of Instantons, arXiv:1408.0012.

[63] E. Gozzi, Functional integral approach to Parisi-Wu stochastic quantization: Scalar theory, Phys.Rev. D28 (1983) 1922-1930.

[64] D. Baumgartner and U. Wenger, Supersymmetric quantum mechanics on the lattice: I. Loop formulation, Nucl.Phys. B894 (2015) 223-253, [arXiv:1412.5393].

[65] M. Mariño, Lectures on non-perturbative effects in large $N$ gauge theories, matrix models and strings, Fortsch. Phys. 62 (2014) 455-540, [arXiv:1206.6272].

[66] G. V. Dunne, Functional determinants in quantum field theory, J. Phys. A41 (2008) 304006, [arXiv:0711.1178].

[67] P. Salomonson and J. van Holten, Fermionic Coordinates and Supersymmetry in Quantum Mechanics, Nucl.Phys. B196 (1982) 509. 
[68] G. Basar, A. Cherman, D. Dorigoni, and M. Ünsal, Volume Independence in the Large $N$ Limit and an Emergent Fermionic Symmetry, Phys.Rev.Lett. 111 (2013), no. 12 121601, [arXiv:1306.2960].

[69] A. Cherman, D. Dorigoni, and M. Ünsal, Neutral bions, renormalons and adiabaticity: Field theory in a box, (unpublished) (2014).

[70] G. V. Dunne, M. Shifman, and M. Ünsal, Infrared Renormalons versus Operator Product Expansions in Supersymmetric and Related Gauge Theories, Phys.Rev.Lett. 114 (2015), no. 19 191601, [arXiv:1502.0668].

[71] E. T. Whittaker and G. N. Watson, A Course of Modern Analysis. Cambridge University Press, fourth ed., 1927. Reprinted 1990.

[72] D. K. Campbell, M. Peyrard, and P. Sodano, Kink - Antikink Interactions in the Double Sine-Gordon Equation, Physica 19D (1986) 165-205.

[73] G. Mussardo, V. Riva, and G. Sotkov, Semiclassical particle spectrum of double Sine-Gordon model, Nucl. Phys. B687 (2004) 189-219, [hep-th/0402179].

[74] M. Ünsal and L. G. Yaffe, Center-stabilized Yang-Mills theory: Confinement and large $N$ volume independence, Phys.Rev. D78 (2008) 065035, [arXiv:0803.0344].

[75] A. Bhoonah, E. Thomas, and A. R. Zhitnitsky, Metastable vacuum decay and $\theta$ dependence in gauge theory. Deformed QCD as a toy model, Nucl.Phys. B890 (2014) 30-47, [arXiv:1407.5121].

[76] J. Ecalle, Les Fonctions Resurgentes, Vol. I-III. Publ. Math. Orsay, 1981.

[77] O. Costin, Asymptotics and Borel Summability. Chapman Hall, 2008.

[78] D. Sauzin, Resurgent functions and splitting problems, RIMS Kokyuroku 1493 (31/05/2006) 48-117 (June, 2007) [arXiv:0706.0137].

[79] I. Aniceto and R. Schiappa, Nonperturbative Ambiguities and the Reality of Resurgent Transseries, arXiv:1308.1115.

[80] M. Marino, R. Schiappa, and M. Weiss, Nonperturbative Effects and the Large-Order Behavior of Matrix Models and Topological Strings, Commun. Num. Theor. Phys. 2 (2008) 349-419, [arXiv:0711.1954].

[81] I. Aniceto, R. Schiappa, and M. Vonk, The Resurgence of Instantons in String Theory, Commun. Num. Theor. Phys. 6 (2012) 339-496, [arXiv:1106.5922].

[82] A. Getmanenko, Resurgent Analysis of the Witten Laplacian in One Dimension, ArXiv e-prints (Sept., 2008) [arXiv:0809.0441].

[83] A. Getmanenko, Resurgent analysis of the Witten Laplacian in one dimension - II, ArXiv e-prints (Apr., 2010) [arXiv:1004.3110].

[84] C. M. Bender and T. T. Wu, Anharmonic oscillator, Phys.Rev. 184 (1969) 1231-1260. 
[85] C. M. Bender and T. Wu, Anharmonic oscillator. 2: A Study of perturbation theory in large order, Phys.Rev. D7 (1973) 1620-1636.

[86] J. J. M. Verbaarschot, P. C. West, and T. T. Wu, Large order behavior of the supersymmetric anharmonic oscillator, Phys. Rev. D42 (1990) 1276-1284.

[87] J. J. M. Verbaarschot and P. C. West, Instantons and Borel resummability for the perturbed supersymmetric anharmonic oscillator, Phys. Rev. D43 (1991) 2718-2725.

[88] E. Witten, A New Look At The Path Integral Of Quantum Mechanics, arXiv: 1009.6032.

[89] G. Guralnik and Z. Guralnik, Complexified path integrals and the phases of quantum field theory, Annals Phys. 325 (2010) 2486-2498, [arXiv:0710.1256].

[90] D. D. Ferrante, G. S. Guralnik, Z. Guralnik, and C. Pehlevan, Complex Path Integrals and the Space of Theories, in Miami 2010: Topical Conference on Elementary Particles, Astrophysics, and Cosmology Fort Lauderdale, Florida, USA, December 14-19, 2010, 2013. arXiv:1301.4233.

[91] A. Jaffe, C. D. Jäkel, and R. E. Martinez, Complex Classical Fields: A Framework for Reflection Positivity, Commun. Math. Phys. 329 (2014) 1-28.

[92] A. Jaffe, C. D. Jäkel, and R. E. Martinez, Complex Classical Fields and Partial Wick Rotations, arXiv:1302.5935.

[93] E. Brezin, J.-C. Le Guillou, and J. Zinn-Justin, Perturbation Theory at Large Order. 2. Role of the Vacuum Instability, Phys.Rev. D15 (1977) 1558-1564.

[94] L. N. Lipatov, Divergence of the Perturbation Theory Series and the Quasiclassical Theory, Sov. Phys. JETP 45 (1977) 216-223. [Zh. Eksp. Teor. Fiz.72,411(1977)].

[95] M. C. Gutzwiller, Periodic orbits and classical quantization conditions, J. Math. Phys. 12 (1971) 343-358.

[96] P. Muratore-Ginanneschi, Path integration over closed loops and Gutzwiller's trace formula, Phys. Rept. 383 (2003) 299-397, [nlin/0210047].

[97] R. Balian and C. Bloch, Solution of the Schrodinger Equation in Terms of Classical Paths, Annals Phys. 85 (1974) 514.

[98] A. Lapedes and E. Mottola, Complex Path Integrals and Finite Temperature, Nucl. Phys. B203 (1982) 58.

[99] R. D. Carlitz and D. A. Nicole, Classical Paths and Quantum Mechanics, Annals Phys. 164 (1985) 411.

[100] J. L. Richard and A. Rouet, Complex Saddle Points in the Double Well Oscillator, Nucl. Phys. B183 (1981) 251. 
[101] J. L. Richard and A. Rouet, Complex Saddle Points Versus Dilute Gas Approximation in the Double Well Anharmonic Oscillator, Nucl. Phys. B185 (1981) 47.

[102] P. A. Millard, Complex Classical Paths and the One-dimensional Sine-Gordon System, Nucl. Phys. B259 (1985) 266.

[103] R. Balian, G. Parisi, and A. Voros, Discrepancies from asymptotic series and their relation to complex classical trajectories, Phys. Rev. Lett. 41 (Oct, 1978) 1141-1144.

[104] R. Balian, G. Parisi, and A. Voros, QUARTIC OSCILLATOR, in In *Marseille 1978, Proceedings, Feynman Path Integrals*, Berlin 1979, 337-360 and Saclay C E N D.PH.T-78-95 (78,REC.DEC) 24p, 1978.

[105] A. Voros, The return of the quartic oscillator. the complex wkb method, Ann. L'Inst. H. Poincare 39 (1983), no. 3 211-338.

[106] C. K. Dumlu and G. V. Dunne, Complex Worldline Instantons and Quantum Interference in Vacuum Pair Production, Phys. Rev. D84 (2011) 125023, [arXiv:1110.1657].

[107] N. Seiberg and E. Witten, Gauge dynamics and compactification to three-dimensions, in The mathematical beauty of physics: A memorial volume for Claude Itzykson. Proceedings, Conference, Saclay, France, June 5-7, 1996, 1996. hep-th/9607163.

[108] N. M. Davies, T. J. Hollowood, V. V. Khoze, and M. P. Mattis, Gluino condensate and magnetic monopoles in supersymmetric gluodynamics, Nucl.Phys. B559 (1999) 123-142, [hep-th/9905015].

[109] E. Poppitz, T. Schäfer, and M. Ünsal, Continuity, Deconfinement, and (Super) Yang-Mills Theory, JHEP 1210 (2012) 115, [arXiv:1205.0290].

[110] I. Affleck, J. A. Harvey, and E. Witten, Instantons and (Super)Symmetry Breaking in (2+1)-Dimensions, Nucl.Phys. B206 (1982) 413.

[111] I. Affleck, M. Dine, and N. Seiberg, Dynamical Supersymmetry Breaking in Supersymmetric QCD, Nucl. Phys. B241 (1984) 493-534.

[112] A. Yung, Instanton Vacuum in Supersymmetric QCD, Nucl.Phys. B297 (1988) 47.

[113] A. M. Din and W. J. Zakrzewski, General Classical Solutions in the $C P^{(n-1)}$ Model, Nucl. Phys. B174 (1980) 397.

[114] C. H. Taubes, The Existence of a Nonminimal Solution to the SU(2) Yang-Mills Higgs Equations on $R^{3}$, Commun. Math. Phys. 86 (1982) 257.

[115] C. H. Taubes, Min-Max Theory for the Yang-Mills Higgs Equations, Commun. Math. Phys. 97 (1985) 473-540.

[116] L. M. Sibner, R. J. Sibner, and K. Uhlenbeck, Solutions to Yang-Mills equations that are not self-dual, Proc. Nat. Acad. Sci. 86 (1989) 8610. 
[117] L. Dallagnol and M. Jardim, Nonsingular complex instantons on euclidean spacetime, Int. J. Geom. Meth. Mod. Phys. 5 (2008) 963-971.

[118] Y. Tanizaki, H. Nishimura, and K. Kashiwa, Evading the sign problem in the mean-field approximation through Lefschetz-thimble path integral, Phys. Rev. D91 (2015), no. 10 101701, [arXiv:1504.0297].

[119] Y. Tanizaki, Y. Hidaka, and T. Hayata, Lefschetz-thimble analysis of the sign problem in one-site fermion model, arXiv:1509.0714.

[120] H. Fujii, S. Kamata, and Y. Kikukawa, Lefschetz thimble structure in one-dimensional lattice Thirring model at finite density, arXiv:1509.0817.

[121] M. Ünsal, Twisted supersymmetric gauge theories and orbifold lattices, JHEP 10 (2006) 089, [hep-th/0603046].

[122] A. Kapustin and E. Witten, Electric-Magnetic Duality And The Geometric Langlands Program, Commun. Num. Theor. Phys. 1 (2007) 1-236, [hep-th/0604151]. 